ESAIM: M2AN 48 (2014) 969-1009

DOI: $10.1051 / \mathrm{m} 2 \mathrm{an} / 2013130$
ESAIM: Mathematical Modelling and Numerical Analysis

www.esaim-m2an.org

\title{
A MIXED FORMULATION OF A SHARP INTERFACE MODEL OF STOKES FLOW WITH MOVING CONTACT LINES*
}

\author{
SHAWN W. WALKER ${ }^{1}$
}

\begin{abstract}
Two-phase fluid flows on substrates (i.e. wetting phenomena) are important in many industrial processes, such as micro-fluidics and coating flows. These flows include additional physical effects that occur near moving (three-phase) contact lines. We present a new 2-D variational (saddlepoint) formulation of a Stokesian fluid with surface tension that interacts with a rigid substrate. The model is derived by an Onsager type principle using shape differential calculus (at the sharp-interface, front-tracking level) and allows for moving contact lines and contact angle hysteresis and pinning through a variational inequality. Moreover, the formulation can be extended to include non-linear contact line motion models. We prove the well-posedness of the time semi-discrete system and fully discrete method using appropriate choices of finite element spaces. A formal energy law is derived for the semi-discrete and fully discrete formulations and preliminary error estimates are also given. Simulation results are presented for a droplet in multiple configurations to illustrate the method.
\end{abstract}

Mathematics Subject Classification. 65N30, 65M12, 76D45, 76M30.

Received December 17, 2012. Revised July 10, 2013

Published online June 30, 2014.

\section{INTRODUCTION}

\subsection{Applications}

In industry, droplet impacting processes play an important role, such as in painting, pesticide application, and the cooling of hot surfaces [22,24,39]. Moreover, the "coating" of solids by films $[29,37,58,60,73]$ is needed in painting/lamination applications, or in the creation of polymer films and metal sheets. All of these examples exhibit a three-phase contact line where the two fluids meet a solid (see Fig. 1). The motion of the contact line can affect the global fluid behavior and introduces a fundamental difficulty in the modeling of these systems. For instance in [15], they performed experimental investigations of the displacement of two immiscible fluids inside a cylindrical capillary. They found that the flow kinematics depends on the direction of displacement and the types of Newtonian fluids. Moreover, the presence or absence of a residual film can also affect the bulk dynamic behavior.

Keywords and phrases. Mixed method, Stokes equations, surface tension, contact line motion, contact line pinning, variational inequality, well-posedness.

* Walker acknowledges funding support from NSF grant DMS-1115636.

1 Department of Mathematics and Center for Computation and Technology, Louisiana State University, Baton Rouge, LA 70803, USA. walker@math.lsu.edu 


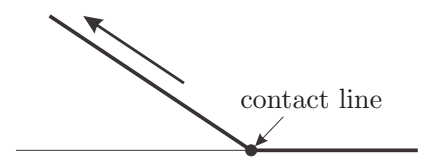

(a) Peeling tape.

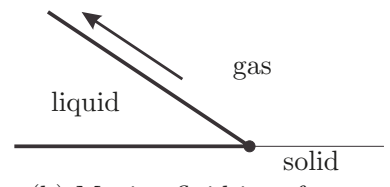

(b) Moving fluid interface.

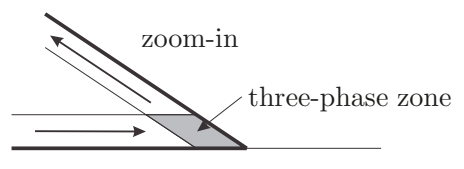

(c) Interfacial flow.

FiguRE 1. Illustration of contact lines and interfacial flows. (a) The contact line (point in 2-D) moves as the tape is pulled away from the substrate. (b) A liquid droplet surrounded by gas that is moving to the left. (c) Close-up of the two-phase interfaces and three-phase zone.

Thus, a fundamental understanding of the dynamic wetting of fluids on surfaces is essential to process control/design (and optimization) in the related industrial applications (e.g. capillary flows in micro-fluidics, etching, electro-chemical treatment of surfaces, etc.). It is not practical to use molecular dynamics simulations for many industrial scale macroscopic fluid problems, so it is necessary to have tractable (continuum) contact line models that incorporate insights from atomistic or molecular dynamic studies. The current paper addresses this issue.

\subsection{Contact line "Paradox"}

There are many types of contact lines that appear in different physical situations (see Fig. 1). The most familiar concerns the peeling of adhesive tape (Fig. 1a) [16,21,68]. Here the contact line (a point in 2-D) separates the tape into two disjoint regions: the part that is still attached to the substrate (where no-slip applies) and the other which is free to deform as a thin flexible body. Typically, one models the free part of the tape as elastic and captures the motion of the contact point with an inequality constraint $[16,68]$. The main thing to note is the contact point is not a material point. So its velocity is not a material velocity. Furthermore, the velocity of the contact line is not necessarily related to the velocity of the material tape. Lastly, it may seem that the velocity of the tape is discontinuous at the contact point. In reality, there is a small (curved) transition region at the contact point from flat to making an angle; ergo, no discontinuity.

The situation is more complicated in the case of two immiscible fluids on a solid substrate. When the fluids are displaced, there arises the classic contact line paradox described in the seminal paper by [41] and addressed by others $[9,10,28,54,55,61]$. In [41], they assumed the wedge-shaped geometry depicted in Figure 1b. By applying free surface boundary conditions on the liquid-gas interface and no-slip conditions on the liquid-solid interface, they obtained a solution to the Navier-Stokes equations that has a logarithmic singularity in the rate of viscous dissipation in a small neighborhood of the moving contact line; clearly, a nonphysical result. The reason is because assuming a wedge-shape geometry and no-slip gives a discontinuity in the velocity boundary condition at the wedge-tip (i.e. the contact line). The singularity is then evident by a standard Sobolev trace theorem that says the $H^{1}$ norm of the (bulk) velocity is unbounded, which implies an infinite rate of viscous dissipation.

There are two ways to remove this singular behavior at the macroscopic level. One is to regularize the shape of the corner region so that the liquid-gas layer smoothly blends into the liquid-solid layer, i.e. a contact angle of $180^{\circ}$. This requires the specification of an effective length scale to smooth the corner. However, this would create a domain shape with a cusp in the other pure fluid phase. This can be problematic (from a PDE point-of-view) for cases where the dynamics of the other fluid is also important. Another way is to modify the model, or introduce a regularization, such that the velocity boundary condition (near the contact line) has no discontinuity. This can be achieved by introducing slip (locally) at the contact line.

\subsection{Summary}

In Section 2, we derive our phenomenological model of a Stokesian fluid coupled to contact line effects via Onsager's variational principle $[50,51]$. Next, we introduce a time-discretization in Section 3 and prove the 
TABLE 1. General notation and symbols.

\begin{tabular}{ccc}
\hline Symbol & Name & Units \\
\hline $\mathbf{u}$ & Vector Fluid Velocity & $\mathrm{m} \mathrm{s}^{-1}$ \\
$\mathbf{x}_{\mathrm{cl}}$ & Contact Line (Point) Location & $\mathrm{m}$ \\
$\theta_{\mathrm{cl}}$ & Contact Angle (Through Fluid) & radians \\
$p$ & Pressure & $\mathrm{N} \mathrm{m}^{-2}$ \\
$\boldsymbol{\sigma}$ & Newtonian Stress Tensor & $\mathrm{N} \mathrm{m}^{-2}$ \\
$\mathbf{f}$ & Gravitational Accel. Vector & $\mathrm{m} \mathrm{s}^{-2}$ \\
$\kappa$ & Total Curvature of $\Gamma_{\mathrm{g}}$ & $\mathrm{m}^{-1}$ \\
$\gamma$ & Surface Tension & $\mathrm{N} \mathrm{m}^{-1}$ \\
$\lambda$ & Contact Line (Point) Pinning Stress & $\mathrm{N} \mathrm{m}^{-1}$ \\
$\boldsymbol{\nu}, \boldsymbol{U n i t}$ Normal, Tangent Vectors of $\Gamma_{\mathrm{g}}$ & - \\
$\Omega$ & Fluid Domain & - \\
$\Gamma_{\mathrm{g}}$ & Liquid-Gas Interface & - \\
$\Gamma_{\mathrm{s}}$ & Liquid-Solid Interface & - \\
$\Gamma_{\mathrm{s}, \mathrm{g}}$ & Solid-Gas Interface & - \\
$\nabla_{\Gamma}$ & Surface Gradient Operator & $\mathrm{m}^{-1}$ \\
$\Delta_{\Gamma}$ & Laplace-Beltrami Operator & $\mathrm{m}^{-2}$ \\
\hline & &
\end{tabular}

well-posedness of the semi-discrete system, followed by proving the stability of a fully discrete approximation scheme in Section 4; see $[25,46,56,57,62,67]$ for other numerical schemes for fluids with contact lines. We then give preliminary error estimates in Section 5 making reasonable regularity assumptions. We conclude in Section 6 with numerical simulations of droplet motion with contact line pinning effects in multiple configurations and discuss future extensions of the formulation.

\section{Phenomenological model of fluids With moving COntaCt Lines}

We develop a computational framework that is both cheap and allows for including simple, and more complicated, models of fluids with contact line dynamics including pinning. The framework is variational via Onsager, and for the purposes of exposition we present the model (in 2-D) for the case of a liquid, gas, and rigid solid phase. See $[27,34,42,48,54,55]$ for other examples using Onsager's principle to derive a model of fluid motion coupled to other physics. A model of electrowetting with "flat" 2-D droplets, with ad hoc modeling of contact line effects, can be found in $[70,72]$.

\subsection{Notation}

Let $\Omega$ be the domain of the liquid bulk, $\Gamma_{\mathrm{g}}$ be the liquid-gas interface, and $\Gamma_{\mathrm{s}}$ be the liquid-solid interface (see Figs. 2 and 3), i.e. $\partial \Omega=\overline{\Gamma_{\mathrm{g}}} \cup \overline{\Gamma_{\mathrm{s}}}, \Gamma_{\mathrm{g}} \cap \Gamma_{\mathrm{s}}=\emptyset$. Table 1 describes the notation we use for the physical domain and the physical variables (e.g. velocity and pressure).

The physical coefficient symbols that appear in the model, as well as their values, are given in Table 2.

\subsection{Droplet pinned to a wall in equilibrium with gravity}

We start with an equilibrium example in order to introduce the shape derivative tools we use to derive the dynamic model with moving contact lines (see Sect. 2.3). Consider the 2-D droplet configuration shown in Figure 2 which is assumed to be in equilibrium. The relevant (free) energy for this problem is

$$
J=\gamma_{\mathrm{s}} \int_{\Gamma_{\mathrm{s}}} 1+\gamma_{\mathrm{g}} \int_{\Gamma_{\mathrm{g}}} 1+\gamma_{\mathrm{s}, \mathrm{g}} \int_{\Gamma_{\mathrm{s}, \mathrm{g}}} 1-\rho \mathbf{f} \cdot \int_{\Omega}\left(\mathbf{x}-\mathbf{x}_{0}\right),
$$

where $\gamma_{i}$ are the surface tension coefficients, $\rho$ is the fluid density, and $\mathbf{f}$ is the gravitational acceleration constant vector. Throughout this paper, we usually omit the $\mathrm{dx}$ notation when writing integrals. 
TABle 2. Physical parameters and values. The solid surface tensions are chosen to give an equilibrium contact angle of $90^{\circ}$.

\begin{tabular}{cccc}
\hline Symbol & Name & Value & Units \\
\hline$\gamma_{\text {water }}$ & Surface Tension of Water/Air & 0.07199 & $\mathrm{~N} \mathrm{~m}^{-1}$ \\
$\gamma_{\mathrm{g}}$ & Surface Tension (liq-gas interface) & $\gamma_{\text {water }}$ & $\mathrm{N} \mathrm{m}^{-1}$ \\
$\gamma_{\mathrm{s}}$ & Surface Tension (liq-sol interface) & $c$ & $\mathrm{~N} \mathrm{~m}^{-1}$ \\
$\gamma_{\mathrm{s}, \mathrm{g}}$ & Surface Tension (sol-gas interface) & $\gamma_{\mathrm{s}}$ & $\mathrm{N} \mathrm{m}^{-1}$ \\
$\mu$ & Dynamic Viscosity & $0.89 \mathrm{E}-3$ & $\mathrm{Kg} \mathrm{m}^{-1} \mathrm{~s}^{-1}$ \\
$\rho$ & Liquid Density & 996.93 & $\mathrm{Kg} \mathrm{m}^{-3}$ \\
$L$ & Length Scale & 0.005 & $\mathrm{~m}^{-1}$ \\
$U_{0}$ & Velocity Scale & 0.02 & $\mathrm{~m} \mathrm{~s}^{-1}$ \\
$t_{0}=L / U_{0}$ & Time Scale & 0.25 & $\mathrm{~seconds}^{\mathrm{s}}$ ) \\
$p_{0}=\gamma_{\text {water }} / L$ & Pressure Scale & 14.398 & $\mathrm{~N} \mathrm{~m}^{-2}$ \\
$\Lambda_{0}=\gamma_{\text {water }} / L$ & Curvature Scale & 14.398 & $\mathrm{~N} \mathrm{~m}^{-2}$ \\
$F_{0}$ & Body Force Scale & 9.81 & $\mathrm{~m} \mathrm{~s}^{-2}$ \\
$\beta_{\mathrm{s}}$ & Slip Coef. (liq-sol) & $1.0 \mathrm{E} 3$ & $\mathrm{~N} \mathrm{~s} \mathrm{~m}^{-3}$ \\
$\beta_{\mathrm{cl}}$ & Viscous Damping Coef. (contact line) & 5.0 & $\mathrm{~N} \mathrm{~s} \mathrm{~m}^{-2}$ \\
$C_{\mathrm{pin}}$ & Pinning Coef. (contact line) & 0.01 & $\mathrm{~N} \mathrm{~m}^{-1}$ \\
\hline \multicolumn{3}{c}{}
\end{tabular}

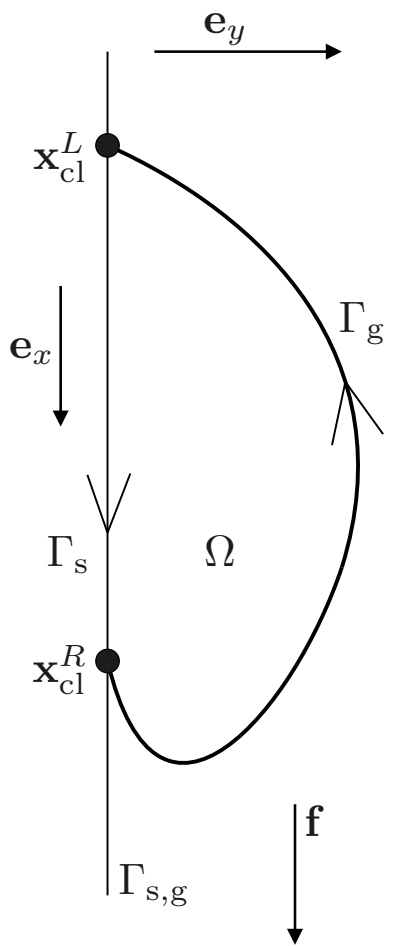

Figure 2. Fluid droplet pinned to a rigid wall in equilibrium with gravity $\mathbf{f}$ (2-D example). The boundary $\partial \Omega$ is positively oriented. The fluid velocity in $\Omega$ vanishes, thus the fluid stress is zero on the solid wall $\Gamma_{\mathrm{s}}$. Hence, the weight of the droplet is supported by a net (pinning) force at the contact points, $\mathbf{x}_{\mathrm{cl}}^{L}, \mathbf{x}_{\mathrm{cl}}^{R}$. Clearly, this pinning force is a "Dirac delta" distribution on $\partial \Omega \equiv \overline{\Gamma_{\mathrm{g}}} \cup \overline{\Gamma_{\mathrm{s}}}$ in the continuum model. 


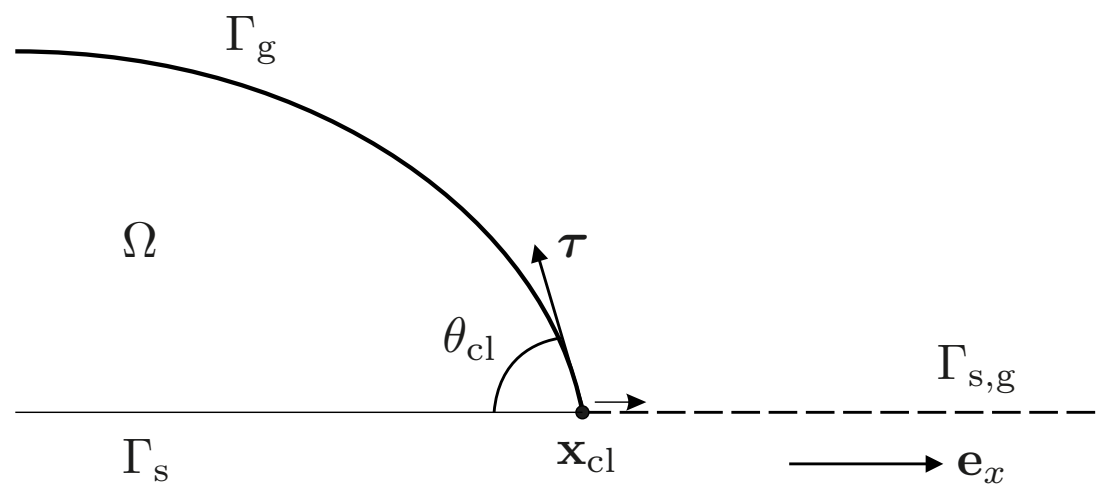

Figure 3. Domain notation with contact line $\mathbf{x}_{\mathrm{cl}}$ moving to the right. $\Omega$ is a liquid domain on top of a solid substrate surrounded by gas. The liquid-solid and liquid-gas boundaries are $\Gamma_{\mathrm{s}}$, $\Gamma_{\mathrm{g}}$. The contact line is moving with speed $\dot{\mathbf{X}}\left(\mathbf{x}_{\mathrm{cl}}\right)$ and the contact angle through the liquid is $\theta_{\mathrm{cl}}$. The unit tangent vector of $\Gamma_{\mathrm{g}}$ is $\tau$. The (non-wet) solid-gas layer is $\Gamma_{\mathrm{s}, \mathrm{g}}$.

To facilitate deriving the equilibrium equations for the shape of the droplet, we introduce the following Lagrangian

$$
\mathcal{L}=J-p_{0}\left(\int_{\Omega} 1-C_{p}\right)+\lambda_{L}\left(\left.\mathbf{x} \cdot \mathbf{e}_{x}\right|_{\mathbf{x}_{\mathrm{cl}}^{L}}-C_{L}\right)+\lambda_{R}\left(\left.\mathbf{x} \cdot \mathbf{e}_{x}\right|_{\mathbf{x}_{\mathrm{cl}}^{R}}-C_{R}\right)
$$

which includes the volume constraint $|\Omega|=C_{p}$ and pinning constraints at the contact points via Lagrange multipliers $p_{0}, \lambda_{L}, \lambda_{R}$.

Let $\mathbf{V}: \mathbb{R}^{2} \rightarrow \mathbb{R}^{2}$ be a smooth perturbation of space that vanishes at a large distance from $\Omega$. We will perturb the domain $\Omega$ with $\mathbf{V}$. Furthermore, we restrict $\mathbf{V}$ such that $\mathbf{V} \cdot \mathbf{e}_{y}=0$ on the rigid wall, i.e. the droplet is constrained to remain on the wall and we are not deforming the wall. Note: $\mathbf{V} \cdot \mathbf{e}_{x}$ on the solid is still free because the contact point constraints are enforced by Lagrange multipliers $\lambda_{L}, \lambda_{R}$.

Next, compute the derivative of $\mathcal{L}$ with respect to domain shape via shape differential calculus [23, 40,64]:

$$
\begin{aligned}
\delta \mathcal{L}(\mathbf{V})= & \gamma_{\mathrm{s}} \int_{\Gamma_{\mathrm{s}}} \nabla_{\Gamma} \cdot \mathbf{V}+\gamma_{\mathrm{g}} \int_{\Gamma_{\mathrm{g}}} \nabla_{\Gamma} \cdot \mathbf{V}+\gamma_{\mathrm{s}, \mathrm{g}} \int_{\Gamma_{\mathrm{s}, \mathrm{g}}} \nabla_{\Gamma} \cdot \mathbf{V}-\rho \mathbf{f} \cdot \int_{\partial \Omega}\left(\mathbf{x}-\mathbf{x}_{0}\right)(\mathbf{V} \cdot \boldsymbol{\nu}) \\
& -p_{0} \int_{\partial \Omega} \mathbf{V} \cdot \boldsymbol{\nu}+\lambda_{L}\left(\left.\mathbf{V} \cdot \mathbf{e}_{x}\right|_{\mathbf{x}_{\mathrm{cl}}^{L}}\right)+\lambda_{R}\left(\left.\mathbf{V} \cdot \mathbf{e}_{x}\right|_{\mathbf{x}_{\mathrm{cl}}^{R}}\right),
\end{aligned}
$$

where the choice of $\mathbf{V}$ respects the corners (contact points) of $\Omega$. The unit normal vector $\boldsymbol{\nu}$ is taken to point outside of $\Omega$. Since the interfaces are 1-D, we have that $\nabla_{\Gamma}=\tau \partial_{s}$ where $\partial_{s}$ is the derivative with respect to arc-length, and $\boldsymbol{\tau}$ is the unit tangent vector of $\partial \Omega$ with positive orientation. So after integration by parts, $(2.3)$ reduces to

$$
\begin{aligned}
\delta \mathcal{L}(\mathbf{V})= & \gamma_{\mathrm{s}}\left[\left.\mathbf{V} \cdot \mathbf{e}_{x}\right|_{\partial \Gamma_{\mathrm{s}}}\right]+\gamma_{\mathrm{g}}\left[\left.\mathbf{V} \cdot \boldsymbol{\tau}\right|_{\partial \Gamma_{\mathrm{g}}}\right]+\gamma_{\mathrm{s}, \mathrm{g}}\left[\left.\mathbf{V} \cdot \mathbf{e}_{x}\right|_{\mathbf{x}_{\mathrm{cl}}^{L}}-\left.\mathbf{V} \cdot \mathbf{e}_{x}\right|_{\mathbf{x}_{\mathrm{cl}}^{R}}\right] \\
& +\gamma_{\mathrm{s}} \int_{\Gamma_{\mathrm{s}}} \kappa_{j} \boldsymbol{\nu} \cdot \mathbf{V}+\gamma_{\mathrm{g}} \int_{\Gamma_{\mathrm{g}}} \kappa_{j} \boldsymbol{\nu} \cdot \mathbf{V}+\gamma_{\mathrm{s}, \mathrm{g}} \int_{\Gamma_{\mathrm{s}, \mathrm{g}}} \kappa_{j} \boldsymbol{\nu} \cdot \mathbf{V}-\rho \mathbf{f} \cdot \int_{\Gamma_{\mathrm{g}}}\left(\mathbf{x}-\mathbf{x}_{0}\right)(\mathbf{V} \cdot \boldsymbol{\nu}) \\
& -p_{0} \int_{\Gamma_{\mathrm{g}}} \mathbf{V} \cdot \boldsymbol{\nu}+\lambda_{L}\left(\left.\mathbf{V} \cdot \mathbf{e}_{x}\right|_{\mathbf{x}_{\mathrm{cl}}^{L}}\right)+\lambda_{R}\left(\left.\mathbf{V} \cdot \mathbf{e}_{x}\right|_{\mathbf{x}_{\mathrm{cl}}^{R}}\right),
\end{aligned}
$$

where $\kappa_{j}$ is the curvature of $\Gamma_{j}(j=g, s,(s, g))$, and $\kappa_{j} \boldsymbol{\nu}=-\left.\partial_{s} \boldsymbol{\tau}\right|_{\Gamma_{j}}$. Note: we shall reserve $\kappa \boldsymbol{\nu}=-\nabla_{\Gamma} \cdot \nabla_{\Gamma} \mathbf{X}$ to refer to the signed total curvature vector of $\Gamma_{\mathrm{g}}$. Accounting for the geometry of $\Gamma_{\mathrm{g}}$ and the restriction on $\mathbf{V}$, 
we arrive at

$$
\begin{aligned}
\delta \mathcal{L}(\mathbf{V})= & \left.\left(\gamma_{\mathrm{s}}-\gamma_{\mathrm{s}, \mathrm{g}}\right) \mathbf{V} \cdot \mathbf{e}_{x}\right|_{\partial \Gamma_{\mathrm{s}}}+\left.\gamma_{\mathrm{g}}\left(\boldsymbol{\tau} \cdot \mathbf{e}_{x}\right) \mathbf{V} \cdot \mathbf{e}_{x}\right|_{\partial \Gamma_{\mathrm{g}}}+\lambda_{L}\left(\left.\mathbf{V} \cdot \mathbf{e}_{x}\right|_{\mathbf{x}_{\mathrm{cl}}^{L}}\right)+\lambda_{R}\left(\left.\mathbf{V} \cdot \mathbf{e}_{x}\right|_{\mathbf{x}_{\mathrm{cl}}^{R}}\right) \\
& +\int_{\Gamma_{\mathrm{g}}}\left(\gamma_{\mathrm{g}} \kappa-\rho \mathbf{f} \cdot\left(\mathbf{x}-\mathbf{x}_{0}\right)-p_{0}\right) \mathbf{V} \cdot \boldsymbol{\nu} .
\end{aligned}
$$

At equilibrium, we must have $\delta \mathcal{L}(\mathbf{V})=0$ for all admissible $\mathbf{V}$. Let $\mathbf{V}=\phi \boldsymbol{\nu}$, where $\phi: \Gamma_{\mathrm{g}} \rightarrow \mathbb{R}$ is a smooth, compact function on $\Gamma_{\mathrm{g}}$, and plug into $(2.5)$

$$
\delta \mathcal{L}(\mathbf{V})=\int_{\Gamma_{\mathrm{g}}}\left(\gamma_{\mathrm{g}} \kappa-\rho \mathbf{f} \cdot\left(\mathbf{x}-\mathbf{x}_{0}\right)-p_{0}\right) \phi=0 \quad \Rightarrow \quad \gamma_{\mathrm{g}} \kappa-\rho \mathbf{f} \cdot\left(\mathbf{x}-\mathbf{x}_{0}\right)-p_{0}=0, \quad \text { on } \Gamma_{\mathrm{g}},
$$

which is the equation that determines the shape of $\Gamma_{\mathrm{g}}$.

Next, noting that $\partial \Gamma_{\mathrm{g}}=\partial \Gamma_{\mathrm{s}}=\left\{\mathbf{x}_{\mathrm{cl}}^{L}, \mathbf{x}_{\mathrm{cl}}^{R}\right\},\left.\right|_{\partial \Gamma_{\mathrm{g}}}=-\left.\right|_{\partial \Gamma_{\mathrm{s}}}$, and $\cos \theta_{\mathrm{cl}}=-\boldsymbol{\tau} \cdot \mathbf{e}_{x}$ at the contact points, (2.5) reduces to

$$
\begin{aligned}
\delta \mathcal{L}(\mathbf{V}) & =\left.\left[\gamma_{\mathrm{g}} \cos \theta_{\mathrm{cl}}+\left(\gamma_{\mathrm{s}}-\gamma_{\mathrm{s}, \mathrm{g}}\right)\right] \mathbf{V} \cdot \mathbf{e}_{x}\right|_{\partial \Gamma_{\mathrm{s}}}+\lambda_{L}\left(\left.\mathbf{V} \cdot \mathbf{e}_{x}\right|_{\mathbf{x}_{\mathrm{cl}}^{L}}\right)+\lambda_{R}\left(\left.\mathbf{V} \cdot \mathbf{e}_{x}\right|_{\mathbf{x}_{\mathrm{cl}}^{R}}\right) \\
& =\left.\left[\gamma_{\mathrm{g}} \cos \theta_{\mathrm{cl}}^{R}+\left(\gamma_{\mathrm{s}}-\gamma_{\mathrm{s}, \mathrm{g}}\right)+\lambda_{R}\right] \mathbf{V} \cdot \mathbf{e}_{x}\right|_{\mathbf{x}_{\mathrm{cl}}^{R}}-\left.\left[\gamma_{\mathrm{g}} \cos \theta_{\mathrm{cl}}^{L}+\left(\gamma_{\mathrm{s}}-\gamma_{\mathrm{s}, \mathrm{g}}\right)-\lambda_{L}\right] \mathbf{V} \cdot \mathbf{e}_{x}\right|_{\mathbf{x}_{\mathrm{cl}}^{L}}
\end{aligned}
$$

Therefore, at equilibrium, suitable choices of the perturbation $\mathbf{V}$ yield

$$
\begin{array}{ll}
\gamma_{\mathrm{g}} \cos \theta_{\mathrm{cl}}^{L}+\left(\gamma_{\mathrm{s}}-\gamma_{\mathrm{s}, \mathrm{g}}\right)-\lambda_{L}=0, & \text { at } \mathbf{x}_{\mathrm{cl}}^{L}, \\
\gamma_{\mathrm{g}} \cos \theta_{\mathrm{cl}}^{R}+\left(\gamma_{\mathrm{s}}-\gamma_{\mathrm{s}, \mathrm{g}}\right)+\lambda_{R}=0, & \text { at } \mathbf{x}_{\mathrm{cl}}^{R}
\end{array}
$$

Remark 2.1. If $\lambda_{L}=\lambda_{R}=0$, then (2.8) is the standard Young's equation for the equilibrium contact angle [53]. The multipliers $\lambda_{L}, \lambda_{R}$ can be interpreted as the net force required to hold the droplet in equilibrium against the gravitational force. From Figure 2, we see that $\lambda_{L}, \lambda_{R}>0$, i.e. $\theta_{\mathrm{cl}}^{L}\left(\theta_{\mathrm{cl}}^{R}\right)$ should be smaller (larger) than the equilibrium values without gravity. In Section 2.3, we will derive a coupled Stokes-contact line model that accounts for pinning through Lagrange multipliers $\lambda_{L}, \lambda_{R}$.

\subsection{Dynamic model derivation via onsager's principle}

We now derive a dynamic (time-dependent) model of a Stokesian droplet with moving contact lines (see Sect. 2.3.7). We use the framework of Onsager's Variational Principle, which says how free energy is dissipated.

\subsubsection{Review}

Onsager's Variational Principle is concerned with physical processes that are not far from equilibrium. It is a method of deriving constitutive laws that we now describe.

Consider a closed mechanical system with free energy $\mathcal{A}(\mathbf{a})$, where $\mathbf{a}$ is a vector representing the configuration (state) variables and is time-dependent. The driving "force" for the evolution of $\mathbf{a}$ is the conservative force $-\nabla_{\mathbf{a}} \mathcal{A}$. If the free energy is a minimum, the system is in a state of stable equilibrium [33], i.e. $\mathbf{a}=\mathbf{0},-\nabla_{\mathbf{a}} \mathcal{A}\left(\mathbf{a}_{0}\right)=\mathbf{0}$, and $\nabla_{\mathbf{a}}^{2} \mathcal{A}\left(\mathbf{a}_{0}\right)$ is positive definite at the critical point $\mathbf{a}_{0}$. Thus, if the system is not in equilibrium at time $t_{0}$, i.e. $\dot{\mathbf{a}}\left(t_{0}\right) \neq \mathbf{0}$, then $-\nabla_{\mathbf{a}} \mathcal{A}\left(\mathbf{a}\left(t_{0}\right)\right) \neq \mathbf{0}$.

The modeling problem is, of course, to determine the connection between $\dot{\mathbf{a}}$ and $-\nabla_{\mathbf{a}} \mathcal{A}(\mathbf{a})$. In the language of continuum mechanics this means to find a constitutive relation between the rates and the conservative forces. Onsager's Principle formalizes this in the following statement:

- The rate of change of the free energy must be balanced by a dissipation functional with respect to perturbation of the rates of change (e.g. $\dot{\mathbf{a}})$. 
TABle 3. Non-dimensional Parameters. All surface tensions are normalized by $\gamma_{\text {water }}$.

\begin{tabular}{ccc}
\hline Symbol & Name & Value \\
\hline $\bar{\gamma}_{\mathrm{g}}=\gamma_{\mathrm{g}} / \gamma_{\text {water }}$ & Surface Tension (liq-gas interface) & 1.0 \\
$\bar{\gamma}_{\mathrm{s}}=\gamma_{\mathrm{s}} / \gamma_{\text {water }}$ & Surface Tension (liq-sol interface) & $\bar{c}$ \\
$\bar{\gamma}_{\mathrm{s}, \mathrm{g}}=\gamma_{\mathrm{s}, \mathrm{g}} / \gamma_{\text {water }}$ & Surface Tension (sol-gas interface) & $\bar{\gamma}_{\mathrm{s}}$ \\
$\mathrm{Re}=\rho U_{0} L / \mu$ & Reynolds Number & $1.120146 \mathrm{E} 2$ \\
$\mathrm{Ca}=\mu U_{0} / \gamma_{\text {water }}$ & Capillary Number & $2.47257 \mathrm{E}-4$ \\
$\mathrm{St}=\rho F_{0} L^{2} /\left(\mu U_{0}\right)$ & Stokes Number & $1.373579 \mathrm{E} 5$ \\
$\bar{\beta}_{\mathrm{s}}=\beta_{\mathrm{s}} U_{0} L / \gamma_{\text {water }}$ & Solid Slip Coef. & 1.389082 \\
$\bar{\beta}_{\mathrm{cl}}=\beta_{\mathrm{cl}} U_{0} / \gamma_{\text {water }}$ & Contact Line Viscous Coef. & 1.389082 \\
$\bar{C}_{\mathrm{pin}}=C_{\text {pin }} / \gamma_{\text {water }}$ & Contact Line Pinning Coef. & 0.1389082 \\
\hline
\end{tabular}

Let $\Phi=\Phi(\dot{\mathbf{a}}, \dot{\mathbf{a}})$ be a dissipation functional. Then Onsager's principle states that

$$
\delta_{\dot{\mathbf{a}}}[\Phi+\dot{\mathcal{A}}]=\delta_{\dot{\mathbf{a}}}\left[\Phi(\dot{\mathbf{a}}, \dot{\mathbf{a}})+\left(\dot{\mathbf{a}} \cdot \nabla_{\mathbf{a}}\right) \mathcal{A}(\mathbf{a})\right]=0, \quad \text { for all } \delta_{\dot{\mathbf{a}}}, \quad \text { where } \mathbf{a} \text { is fixed. }
$$

Thus, the modeling problem is reduced to determining the specific dissipation functional. Fundamental thermodynamic considerations demand that $\Phi$ be a positive definite (semi-definite), symmetric function of its two arguments $[50,51]$. Since the processes are inherently assumed to be near equilibrium, typically $\Phi$ is assumed to be quadratic. Within these restrictions, $\Phi$ can be anything, e.g. it may have variable coefficients. Several other authors have developed variational principles involving dissipation in other contexts such as viscous flow and polymer solutions $[27,34,42,48,54,55]$. Ultimately, Onsager's Principle is an assumption we use to derive the model for our physical system.

Remark 2.2. "Dissipation" is a rough approximation of the aggregate effect of many molecules and atoms interacting, e.g. energy is "dissipated" amongst many atoms through pairwise interactions, collisions, etc. The dissipation functional $\Phi$ is a convenient idealization that allows one to ignore the details of molecular interactions, i.e. another way of developing constitutive laws. It is nothing more than that.

\subsubsection{Configuration variables}

The liquid domain $\Omega$ (i.e. droplet shape) is of fundamental importance in this problem. In particular, we have the droplet interface $\partial \Omega=\overline{\Gamma_{\mathrm{g}}} \cup \overline{\Gamma_{\mathrm{s}}}$, and the $x$-axis partitions as $(-\infty, \infty)=\overline{\Gamma_{\mathrm{s}}} \cup \Gamma_{\mathrm{s}, \mathrm{g}}$. We also have the contact points $\left\{\mathbf{x}_{\mathrm{cl}}^{L}, \mathbf{x}_{\mathrm{cl}}^{R}\right\}=\overline{\Gamma_{\mathrm{g}}} \cap \overline{\Gamma_{\mathrm{s}}}$.

We can, equivalently, represent the interface shape by an explicit parametrization $\mathbf{X}(t, \cdot): \partial \Omega(t) \rightarrow \mathbb{R}^{2}$, i.e. the identity map $\mathbf{X}(t, \partial \Omega(t))=\partial \Omega(t)$ that is assumed to be positively oriented. In other words, the definition of $\Omega$ depends on its boundary. Note that $\Omega$ has corners at the contact line which are related to the parametrization in the obvious way $\mathbf{x}_{\mathrm{cl}}^{L}(t)=\mathbf{X}\left(t, \mathbf{x}_{\mathrm{cl}}^{L}\right), \mathbf{x}_{\mathrm{cl}}^{R}(t)=\mathbf{X}\left(t, \mathbf{x}_{\mathrm{cl}}^{R}\right)$.

The fundamental configuration variable (i.e. a) of the system is the position of all liquid particles (i.e. $\bar{\Omega}$ ), which we label by the coordinates $\mathbf{x}$. The rates of change of $\mathbf{x}$ are given by the liquid velocity $\mathbf{u}$ in $\Omega$ and the rate of change of position of the interface $\dot{\mathbf{X}}$. Note that $\dot{\mathbf{X}}$ and $\mathbf{u}$ are not independent and are connected through appropriate constraints (Sect. 2.3.4).

All variables are taken to be dimensionless and Table 3 lists the non-dimensional parameters in the model. We include the Reynolds number Re (even though we only obtain the Stokes equations) so we can add back fluid inertial effects at a later time (Sect. 6.1.1).

\subsubsection{Functionals}

The free energy functional of the droplet system is $\widetilde{\mathcal{A}}=\mu U_{0} L^{2} \mathcal{A}$, where $\mathcal{A}$ is dimensionless and is defined as

$$
\mathcal{A}=-\operatorname{St} \int_{\Omega} \mathbf{f} \cdot\left(\mathbf{x}-\mathbf{x}_{0}\right)+\frac{1}{\mathrm{Ca}}\left(\int_{\Gamma_{\mathrm{s}}} \bar{\gamma}_{\mathrm{s}}+\int_{\Gamma_{\mathrm{g}}} 1+\int_{\Gamma_{\mathrm{s}, \mathrm{g}}} \bar{\gamma}_{\mathrm{s}, \mathrm{g}}\right),
$$


where $\mathbf{x}_{0}$ is a reference point and the surface tension coefficients are variable. $\mathcal{A}$ is the potential energy of the system and is the dimensionless version of (2.1). We assume the surface tension coefficients $\bar{\gamma}_{\mathrm{s}}, \bar{\gamma}_{\mathrm{s}, \mathrm{g}}$ are functions of $x=\mathbf{x} \cdot \mathbf{e}_{x}$ but independent of time (i.e. $\left.\partial_{t} \bar{\gamma}_{\mathrm{s}}=\partial_{t} \bar{\gamma}_{\mathrm{s}, \mathrm{g}}=0\right)$. This models a solid surface with a known chemical pattern. Note that $\bar{\gamma}_{\mathrm{g}}=1$ in non-dimensional units.

Remark 2.3. Often an additional term is included in the free energy functional in diffuse interface models, i.e. the double-well potential. This is done to stabilize the interface between the two immiscible fluids so that they do not mix. This is not required here because we represent the interface explicitly by the function $\mathbf{X}$. In other words, the two phases cannot mix because the parametrization explicitly enforces the separation of the two phases.

The corresponding dissipation functional is $\widetilde{\Phi}=\gamma_{\text {water }} U_{0} L \Phi$, where $\Phi$ is dimensionless and is

$$
\Phi=\int_{\Gamma_{\mathrm{s}}} \frac{\bar{\beta}_{\mathrm{s}}}{2}(\mathbf{u} \cdot \boldsymbol{\tau})^{2}+\left.\left(\frac{\bar{\beta}_{\mathrm{cl}}}{2}\left(\dot{\mathbf{X}} \cdot \mathbf{e}_{x}\right)^{2}\right)\right|_{\mathbf{x}_{\mathrm{cl}}^{L}}+\left.\left(\frac{\bar{\beta}_{\mathrm{cl}}}{2}\left(\dot{\mathbf{X}} \cdot \mathbf{e}_{x}\right)^{2}\right)\right|_{\mathbf{x}_{\mathrm{cl}}^{R}}+\mathrm{Ca} \int_{\Omega} \frac{1}{4} D(\mathbf{u}): D(\mathbf{u})
$$

where we used the fact that evaluation at the contact line is viewed as an integral along the contact line, so it has units of length. Note: the surrounding gas dynamics are ignored, and the solid substrate is assumed perfectly rigid. The first term will eventually yield a slip boundary condition with parameter $\bar{\beta}_{\mathrm{s}}$. The contact line terms model energy dissipation due to the velocity of the contact line, where $\bar{\beta}_{\mathrm{cl}}$ is the contact line viscous friction coefficient. The last term is the total rate of viscous dissipation in the bulk $\Omega$.

\subsubsection{Constraints}

We make the following reasonable assumptions on the system. The solid wall does not move and the fluid does not penetrate into the solid. Moreover, no cavitation is possible (i.e. pockets of air between the solid and fluid) and $\Gamma_{\mathrm{g}}$ adheres to the fluid domain $\Omega$. In mathematical terms, we have

$$
(\dot{\mathrm{X}}-\mathbf{u}) \cdot \boldsymbol{\nu}=0, \quad \text { on } \Gamma_{\mathrm{g}}, \quad \dot{\mathrm{X}} \cdot \boldsymbol{\nu}=0, \quad \mathbf{u} \cdot \boldsymbol{\nu}=0, \quad \text { on } \Gamma_{\mathrm{s}} .
$$

Remark 2.4. The tangential component of $\dot{\mathbf{X}}$ does not affect the shape of $\Omega$. It is only a re-parametrization of the boundary. Furthermore, the constraints on $\dot{\mathbf{X}}$ respect the corners of $\Omega$ (i.e. the contact points). This is crucial for allowing computation of shape derivatives [23,64].

The remaining physical constraints are divergence free velocity

$$
\nabla \cdot \mathbf{u}=0, \text { in } \Omega,
$$

and an additional pinning force at the contact line that satisfies a complementarity condition

$$
|\lambda| \leq \bar{C}_{\text {pin }},\left.\quad\left(\lambda-\bar{C}_{\text {pin }}\right)\left(\lambda+\bar{C}_{\text {pin }}\right)\left(\dot{\mathbf{X}} \cdot \mathbf{e}_{x}\right)\right|_{\mathbf{x}_{\mathrm{cl}}}=0
$$

This is a basic model of static Coulombic friction, i.e. it models pinning and release of the contact line, which can be written more succinctly as

$$
\lambda=\bar{C}_{\text {pin }} \operatorname{sgn}\left(\left.\dot{\mathbf{X}} \cdot \mathbf{e}_{x}\right|_{\mathbf{x}_{\mathrm{cl}}}\right)
$$




\subsubsection{Lagrangian}

In order to perform the minimization in $(2.9)$ with constraints, we formulate a Lagrangian $\widetilde{\mathcal{L}}=\gamma_{\text {water }} U_{0} L \mathcal{L}$, where $\mathcal{L}$ is dimensionless. For given $\Omega$, define $\mathcal{L}$ by

$$
\begin{aligned}
\mathcal{L}(\Omega, \mathbf{u}, \dot{\mathbf{X}})= & \Phi+\operatorname{Ca} \dot{\mathcal{A}}-\int_{\Omega} p(\nabla \cdot \mathbf{u}-0)-\int_{\Gamma_{\mathrm{g}}} \Lambda_{g}(\dot{\mathbf{X}}-\mathbf{u}) \cdot \boldsymbol{\nu} \\
& +\lambda_{L}\left(\left.\dot{\mathbf{X}} \cdot \mathbf{e}_{x}\right|_{\mathbf{x}_{\mathrm{cl}}^{L}}-0\right)+\lambda_{R}\left(\left.\dot{\mathbf{X}} \cdot \mathbf{e}_{x}\right|_{\mathbf{x}_{\mathrm{cl}}^{R}}-0\right),
\end{aligned}
$$

which is a function of the rates $\mathbf{u}, \dot{\mathbf{X}}$ and can be varied independently. No restrictions are placed on the multipliers $p, \Lambda_{g}$ and are allowed to take on whatever values necessary to enforce their associated constraints. But the pinning values must satisfy $\left|\lambda_{L}\right|,\left|\lambda_{R}\right| \leq \bar{C}_{\text {pin }}$, thus their associated constraints are only enforced when $\left|\lambda_{L}\right|,\left|\lambda_{R}\right|<\bar{C}_{\text {pin }}$.

Remark 2.5 (interpretation of the multipliers). We will see later that $p$ is the fluid pressure, $\Lambda_{g}$ is the curvature of $\Gamma_{\mathrm{g}}$, and $\lambda_{L}, \lambda_{R}$ are static, "Coulombic" friction forces present at the contact line. But our formulation of the constraints does not depend on the fine physical details. For instance, the true pressure is caused by the fluid material having an extremely small amount of compressibility yet we only model the end result (incompressibility) by imposing it as a constraint. How it became incompressible is not important.

Similarly, contact line pinning is a directly observed effect in fluid droplets interacting with solid substrates, but the physical reasons are rather complicated and not completely understood; $c f$. the case of modeling dry friction with Coulombic friction. Our static friction model is ultimately a phenomenological rule for describing the approximate outcome of an extremely complicated physical interaction. The main advantage of the approximation is its simplicity and flexibility.

The constraints $\dot{\mathbf{X}} \cdot \boldsymbol{\nu}=\mathbf{u} \cdot \boldsymbol{\nu}=0$ on $\Gamma_{\mathrm{s}}$ are enforced explicitly (i.e. just plug them in) because the solid surface is flat. The same can be done for a curved solid, or one can introduce multipliers to enforce these constraints.

\subsubsection{Rate of change of the free energy}

We first compute the rate of change of the free energy. For simplicity, assume $\mathbf{f}$ is a constant vector, and recall that $\partial_{t} \bar{\gamma}_{\mathrm{s}}=\partial_{t} \bar{\gamma}_{\mathrm{s}, \mathrm{g}}=0$. Using shape differential calculus $[23,40,64]$, the dimensional rate of change is $\mathrm{d} \widetilde{\mathcal{A}} / \mathrm{d} t=\left(\mu U_{0} L^{2} / t_{0}\right) \dot{\mathcal{A}}$, where $\dot{\mathcal{A}}$ is dimensionless, is given by

$$
\begin{aligned}
\dot{\mathcal{A}}= & -\mathrm{St} \int_{\partial \Omega} \mathbf{f} \cdot\left(\mathbf{x}-\mathbf{x}_{0}\right)(\mathbf{V} \cdot \boldsymbol{\nu})+\frac{1}{\mathrm{Ca}}\left\{\int_{\Gamma_{\mathrm{s}}}(\mathbf{V} \cdot \nabla) \bar{\gamma}_{\mathrm{s}}+\int_{\Gamma_{\mathrm{s}}} \bar{\gamma}_{\mathrm{s}} \nabla_{\Gamma} \mathbf{X}: \nabla_{\Gamma} \mathbf{V}\right. \\
& \left.+\int_{\Gamma_{\mathrm{g}}} \nabla_{\Gamma} \mathbf{X}: \nabla_{\Gamma} \mathbf{V}+\int_{\Gamma_{\mathrm{s}, \mathrm{g}}}(\mathbf{V} \cdot \nabla) \bar{\gamma}_{\mathrm{s}, \mathrm{g}}+\int_{\Gamma_{\mathrm{s}, \mathrm{g}}} \bar{\gamma}_{\mathrm{s}, \mathrm{g}} \nabla_{\Gamma} \mathbf{X}: \nabla_{\Gamma} \mathbf{V}\right\}
\end{aligned}
$$

where $\nabla_{\Gamma}=\tau \partial_{s}$ and $\mathbf{V} \equiv \dot{\mathbf{X}}$ is the velocity of deformation of the domain $\Omega$ (recall Sect. 2.2). Note that $\dot{\mathbf{X}} \cdot \boldsymbol{\nu}=\mathbf{u} \cdot \boldsymbol{\nu}$ on $\partial \Omega$. Thus, we can rewrite the body force term as

$$
\int_{\partial \Omega} \mathbf{f} \cdot\left(\mathbf{x}-\mathbf{x}_{0}\right)(\mathbf{u} \cdot \boldsymbol{\nu})=\int_{\Omega}(\nabla \cdot \mathbf{u})\left[\mathbf{f} \cdot\left(\mathbf{x}-\mathbf{x}_{0}\right)\right]+\int_{\Omega}[(\mathbf{u} \cdot \nabla) \mathbf{f}] \cdot\left(\mathbf{x}-\mathbf{x}_{0}\right)+\int_{\Omega}[(\mathbf{u} \cdot \nabla) \mathbf{x}] \cdot \mathbf{f}=\int_{\Omega} \mathbf{f} \cdot \mathbf{u},
$$

because $\nabla \cdot \mathbf{u}=0$. Combining, we have that $\dot{\mathcal{A}}$ is a functional depending explicitly on $\mathbf{u}, \dot{\mathbf{X}}$ :

$$
\begin{aligned}
\dot{\mathcal{A}}(\mathbf{u}, \dot{\mathbf{X}})= & -\operatorname{St} \int_{\Omega} \mathbf{f} \cdot \mathbf{u}+\frac{1}{\mathrm{Ca}}\left\{\int_{\Gamma_{\mathrm{s}}}(\dot{\mathbf{X}} \cdot \nabla) \bar{\gamma}_{\mathrm{s}}+\int_{\Gamma_{\mathrm{s}}} \bar{\gamma}_{\mathrm{s}} \partial_{s} \mathbf{X} \cdot \partial_{s} \dot{\mathbf{X}}\right. \\
& \left.+\int_{\Gamma_{\mathrm{g}}} \partial_{s} \mathbf{X} \cdot \partial_{s} \dot{\mathbf{X}}+\int_{\Gamma_{\mathrm{s}, \mathrm{g}}}(\dot{\mathbf{X}} \cdot \nabla) \bar{\gamma}_{\mathrm{s}, \mathrm{g}}+\int_{\Gamma_{\mathrm{s}, \mathrm{g}}} \bar{\gamma}_{\mathrm{s}, \mathrm{g}} \partial_{s} \mathbf{X} \cdot \partial_{s} \dot{\mathbf{X}}\right\} .
\end{aligned}
$$




\subsubsection{Sensitivity of the Lagrangian (weak formulation)}

The weak formulation of the governing equations for the dynamic droplet model, with moving contact lines, derives from setting the first variation of (2.16) to zero (with respect to the rates). More precisely, define the following perturbations. Let $\mathbf{v}$ be a perturbation of $\mathbf{u}$ such that $\mathbf{v} \cdot \mathbf{e}_{y}=0$ on $\Gamma_{\mathrm{s}}$ and $\mathbf{v}$ is smooth. Next, let $\mathbf{Y}$ be a perturbation of $\dot{\mathbf{X}}$ such that $\mathbf{Y} \cdot \mathbf{e}_{y}=0$ on $\overline{\Gamma_{\mathrm{s}}} \cup \overline{\Gamma_{\mathrm{s}, \mathrm{g}}}$ and $\mathbf{Y}$ is smooth. Similarly, let $q, \mu$ be smooth perturbations of $p, \Lambda_{g}$ respectively. Then the (formal) weak formulation is: for each $t \in[0, T]$, find $(\mathbf{u}, \dot{\mathbf{X}})$ and $\left(p, \Lambda_{g}, \lambda_{L}, \lambda_{R}\right)$ such that for all admissible perturbations the following is satisfied:

$$
\begin{aligned}
\delta_{\mathbf{u}} \mathcal{L}(\Omega, \mathbf{u}, \dot{\mathbf{X}} ; \mathbf{v})= & \mathrm{Ca} \int_{\Omega} \frac{1}{2} D(\mathbf{u}): D(\mathbf{v})-\int_{\Omega} p \nabla \cdot \mathbf{v}+\int_{\Gamma_{\mathrm{s}}} \bar{\beta}_{\mathrm{s}}(\mathbf{u} \cdot \boldsymbol{\tau})(\mathbf{v} \cdot \boldsymbol{\tau}) \\
& +\int_{\Gamma_{\mathrm{g}}} \Lambda_{g} \mathbf{v} \cdot \boldsymbol{\nu}-\mathrm{StCa} \int_{\Omega} \mathbf{f} \cdot \mathbf{v}=0, \quad \text { for all } \mathbf{v}, \\
\delta_{\dot{\mathbf{X}}} \mathcal{L}(\Omega, \mathbf{u}, \dot{\mathbf{X}} ; \mathbf{Y})= & \left.\bar{\beta}_{\mathrm{cl}}\left(\dot{\mathbf{X}} \cdot \mathbf{e}_{x}\right) \mathbf{Y} \cdot \mathbf{e}_{x}\right|_{\mathbf{x}_{\mathrm{cl}}^{L}}+\left.\bar{\beta}_{\mathrm{cl}}\left(\dot{\mathbf{X}} \cdot \mathbf{e}_{x}\right) \mathbf{Y} \cdot \mathbf{e}_{x}\right|_{\mathbf{x}_{\mathrm{cl}}^{R}}+\left.\lambda_{L} \mathbf{Y} \cdot \mathbf{e}_{x}\right|_{\mathbf{x}_{\mathrm{cl}}^{L}}+\left.\lambda_{R} \mathbf{Y} \cdot \mathbf{e}_{x}\right|_{\mathbf{x}_{\mathrm{cl}}^{R}} \\
& +\int_{\Gamma_{\mathrm{s}}}(\mathbf{Y} \cdot \boldsymbol{\tau}) \partial_{s} \bar{\gamma}_{\mathrm{s}}+\int_{\Gamma_{\mathrm{s}}} \bar{\gamma}_{\mathrm{s}} \partial_{s} \mathbf{X} \cdot \partial_{s} \mathbf{Y}+\int_{\Gamma_{\mathrm{s}, \mathrm{g}}}(\mathbf{Y} \cdot \boldsymbol{\tau}) \partial_{s} \bar{\gamma}_{\mathrm{s}, \mathrm{g}}+\int_{\Gamma_{\mathrm{s}, \mathrm{g}}} \bar{\gamma}_{\mathrm{s}, \mathrm{g}} \partial_{s} \mathbf{X} \cdot \partial_{s} \mathbf{Y} \\
& -\int_{\Gamma_{\mathrm{g}}} \Lambda_{g} \mathbf{Y} \cdot \boldsymbol{\nu}+\int_{\Gamma_{\mathrm{g}}} \partial_{s} \mathbf{X} \cdot \partial_{s} \mathbf{Y}=0, \quad \text { for all } \mathbf{Y}, \\
\delta_{p} \mathcal{L}(\Omega, \mathbf{u}, \dot{\mathbf{X}} ; q)= & -\int_{\Omega} q \nabla \cdot \mathbf{u}=0, \quad \text { for all } q, \\
\delta_{\Lambda_{g}} \mathcal{L}(\Omega, \mathbf{u}, \dot{\mathbf{X}} ; \mu)= & -\int_{\Gamma_{\mathrm{g}}} \mu(\dot{\mathbf{X}}-\mathbf{u}) \cdot \boldsymbol{\nu}=0, \quad \text { for all } \mu
\end{aligned}
$$

where $\Omega(t)$ and $\mathbf{X}(t)$ is held fixed. The pinning multipliers are determined by (2.15) which can be written as a variational inequality: find $\lambda_{L}, \lambda_{R}$ in $\left[-\bar{C}_{\text {pin }}, \bar{C}_{\text {pin }}\right]$ such that

$$
\left.\left(\xi-\lambda_{L}\right)\left(\dot{\mathbf{X}} \cdot \mathbf{e}_{x}\right)\right|_{\mathbf{x}_{\mathrm{cl}}^{L}} \leq 0,\left.\quad\left(\xi-\lambda_{R}\right)\left(\dot{\mathbf{X}} \cdot \mathbf{e}_{x}\right)\right|_{\mathbf{x}_{\mathrm{cl}}^{R}} \leq 0, \quad \text { for all } \xi \text { in }\left[-\bar{C}_{\text {pin }}, \bar{C}_{\text {pin }}\right] \subset \mathbb{R} .
$$

\subsubsection{Recover strong form equations}

Clearly, we recover the constraints in Section 2.3.4. Next, consider $\delta_{\dot{\mathbf{X}}} \mathcal{L}(\Omega, \mathbf{u}, \dot{\mathbf{X}} ; \mathbf{Y})=0$ for all $\mathbf{Y}$ such that $\mathbf{Y} \cdot \mathbf{e}_{y}=0$ on $\overline{\Gamma_{\mathrm{s}}} \cup \overline{\Gamma_{\mathrm{s}, \mathrm{g}}}$. Then integration by parts on the interfaces gives

$$
\begin{aligned}
0= & \left.\bar{\beta}_{\mathrm{cl}}\left(\dot{\mathbf{X}} \cdot \mathbf{e}_{x}\right) \mathbf{Y} \cdot \mathbf{e}_{x}\right|_{\mathbf{x}_{\mathrm{cl}}^{L}}+\left.\bar{\beta}_{\mathrm{cl}}\left(\dot{\mathbf{X}} \cdot \mathbf{e}_{x}\right) \mathbf{Y} \cdot \mathbf{e}_{x}\right|_{\mathbf{x}_{\mathrm{cl}}^{R}}+\left.\lambda_{L} \mathbf{Y} \cdot \mathbf{e}_{x}\right|_{\mathbf{x}_{\mathrm{cl}}^{L}}+\left.\lambda_{R} \mathbf{Y} \cdot \mathbf{e}_{x}\right|_{\mathbf{x}_{\mathrm{cl}}^{R}} \\
& +\left.\bar{\gamma}_{\mathrm{s}} \mathbf{e}_{x} \cdot \mathbf{Y}\right|_{\partial \Gamma_{\mathrm{s}}}+\left.\boldsymbol{\tau} \cdot \mathbf{Y}\right|_{\partial \Gamma_{\mathrm{g}}}+\left.\bar{\gamma}_{\mathrm{s}, \mathrm{g}} \mathbf{e}_{x} \cdot \mathbf{Y}\right|_{\partial \Gamma_{\mathrm{s}, \mathrm{g}}} \\
& -\int_{\Gamma_{\mathrm{g}}} \Lambda_{g} \mathbf{Y} \cdot \boldsymbol{\nu}-\int_{\Gamma_{\mathrm{s}}} \bar{\gamma}_{\mathrm{s}} \partial_{s} \boldsymbol{\tau} \cdot \mathbf{Y}-\int_{\Gamma_{\mathrm{g}}} \partial_{s} \boldsymbol{\tau} \cdot \mathbf{Y}-\int_{\Gamma_{\mathrm{s}, \mathrm{g}}} \bar{\gamma}_{\mathrm{s}, \mathrm{g}} \partial_{s} \boldsymbol{\tau} \cdot \mathbf{Y}
\end{aligned}
$$

Since $\kappa_{j} \boldsymbol{\nu}=-\partial_{s} \boldsymbol{\tau}$ on $\Gamma_{j}$, we have

$$
\begin{aligned}
0= & \left.\bar{\beta}_{\mathrm{cl}}\left(\dot{\mathbf{X}} \cdot \mathbf{e}_{x}\right) \mathbf{Y} \cdot \mathbf{e}_{x}\right|_{\mathbf{x}_{\mathrm{cl}}^{L}}+\left.\bar{\beta}_{\mathrm{cl}}\left(\dot{\mathbf{X}} \cdot \mathbf{e}_{x}\right) \mathbf{Y} \cdot \mathbf{e}_{x}\right|_{\mathbf{x}_{\mathrm{cl}}^{R}}+\left.\lambda_{L} \mathbf{Y} \cdot \mathbf{e}_{x}\right|_{\mathbf{x}_{\mathrm{cl}}^{L}}+\left.\lambda_{R} \mathbf{Y} \cdot \mathbf{e}_{x}\right|_{\mathbf{x}_{\mathrm{cl}}^{R}} \\
& +\left.\bar{\gamma}_{\mathrm{s}} \mathbf{e}_{x} \cdot \mathbf{Y}\right|_{\partial \Gamma_{\mathrm{s}}}+\left.\boldsymbol{\tau} \cdot \mathbf{Y}\right|_{\partial \Gamma_{\mathrm{g}}}+\left.\bar{\gamma}_{\mathrm{s}, \mathrm{g}} \mathbf{e}_{x} \cdot \mathbf{Y}\right|_{\partial \Gamma_{\mathrm{s}, \mathrm{g}}}-\int_{\Gamma_{\mathrm{g}}} \Lambda_{g} \mathbf{Y} \cdot \boldsymbol{\nu}+\int_{\Gamma_{\mathrm{g}}} \kappa \boldsymbol{\nu} \cdot \mathbf{Y} .
\end{aligned}
$$

Suppose $\mathbf{Y}=\phi \boldsymbol{\nu}$, where $\phi$ is smooth and has compact support on $\Gamma_{\mathrm{g}}$. Then

$$
0=\int_{\Gamma_{\mathrm{g}}}\left(\kappa-\Lambda_{g}\right) \phi, \quad \forall \phi, \quad \Rightarrow \quad \Lambda_{g}=\kappa
$$


Next, note the interface boundary relations

$$
-\left.\right|_{\partial \Gamma_{\mathrm{g}}}=\left.\right|_{\partial \Gamma_{\mathrm{s}}}=\left.\right|_{\mathbf{x}_{\mathrm{cl}}^{R}}-\left.\right|_{\mathbf{x}_{\mathrm{cl}}^{L}},\left.\quad\right|_{\partial \Gamma_{\mathrm{s}, \mathrm{g}}}=\left.\right|_{\mathbf{x}_{\mathrm{cl}}^{L}}-\left.\right|_{\mathbf{x}_{\mathrm{cl}}^{R}} \cdot
$$

Choosing a smooth test function such that $\mathbf{Y}=\mathbf{e}_{x}$ at $\mathbf{x}_{\mathrm{cl}}^{L}$ and $\mathbf{Y}=\mathbf{0}$ at $\mathbf{x}_{\mathrm{cl}}^{R}$, and vice versa, we get

$$
\begin{array}{ll}
0=\bar{\beta}_{\mathrm{cl}}\left(\dot{\mathbf{X}} \cdot \mathbf{e}_{x}\right)+\lambda_{L}-\bar{\gamma}_{\mathrm{s}}+\boldsymbol{\tau} \cdot \mathbf{e}_{x}+\bar{\gamma}_{\mathrm{s}, \mathrm{g}}, & \text { at } \mathbf{x}_{\mathrm{cl}}^{L}, \\
0=\bar{\beta}_{\mathrm{cl}}\left(\dot{\mathbf{X}} \cdot \mathbf{e}_{x}\right)+\lambda_{R}+\bar{\gamma}_{\mathrm{s}}-\boldsymbol{\tau} \cdot \mathbf{e}_{x}-\bar{\gamma}_{\mathrm{s}, \mathrm{g}}, & \text { at } \mathbf{x}_{\mathrm{cl}}^{R} .
\end{array}
$$

where $\lambda_{L}, \lambda_{R}$ is determined by (2.19). Because $-\boldsymbol{\tau} \cdot \mathbf{e}_{x}=\cos \theta_{\mathrm{cl}}$, we obtain a modified Young's relation at the contact line

$$
\cos \theta_{\mathrm{cl}}+\bar{\gamma}_{\mathrm{s}}-\bar{\gamma}_{\mathrm{s}, \mathrm{g}}=\mathrm{S}\left(\bar{\beta}_{\mathrm{cl}}\left(\dot{\mathbf{X}} \cdot \mathbf{e}_{x}\right)+\lambda\right), \quad \text { on }\left\{\mathbf{x}_{\mathrm{cl}}^{L}, \mathbf{x}_{\mathrm{cl}}^{R}\right\},
$$

where

$$
\mathrm{S}=\left\{\begin{array}{r}
1, \text { at } \mathbf{x}_{\mathrm{cl}}^{L}, \\
-1, \text { at } \mathbf{x}_{\mathrm{cl}}^{R} .
\end{array} \quad \lambda=\left\{\begin{array}{l}
\lambda_{L}, \text { at } \mathbf{x}_{\mathrm{cl}}^{L}, \\
\lambda_{R}, \text { at } \mathbf{x}_{\mathrm{cl}}^{R} .
\end{array}\right.\right.
$$

Remark 2.6 (Modeling of Contact Line Motion). Note that the contact angle is dependent on the bulk hydrodynamics via $\dot{\mathbf{X}}\left(\mathrm{x}_{\mathrm{cl}}\right) \cdot \mathbf{e}_{x}$ in (2.23), because $\dot{\mathbf{X}}$ is coupled to the fluid velocity through (2.12). Equation (2.23) is a modification of the standard static force balance (i.e. when right-hand-side is zero) that includes nonequilibrium, dissipative effects. Note that when the droplet moves in the positive $\mathbf{e}_{x}$ direction, $\lambda$ is positive at both contact points which decreases (increases) the contact angle at $\mathbf{x}_{\mathrm{cl}}^{L}\left(\mathbf{x}_{\mathrm{cl}}^{R}\right)$, so is consistent with experience.

Now, consider $\delta_{\mathbf{u}} \mathcal{L}(\Omega, \mathbf{u}, \dot{\mathbf{X}} ; \mathbf{v})=0$ for all $\mathbf{v}$ such that $\mathbf{v} \cdot \mathbf{e}_{y}=0$ on $\overline{\Gamma_{\mathrm{s}}} \cup \overline{\Gamma_{\mathrm{s}, \mathrm{g}}}$. Then integration by parts on $\Omega$ gives

$$
0=-\int_{\Omega}[\nabla \cdot \boldsymbol{\sigma}] \cdot \mathbf{v}+\int_{\partial \Omega} \mathbf{v} \cdot \boldsymbol{\sigma} \boldsymbol{\nu}+\int_{\Gamma_{\mathrm{s}}} \bar{\beta}_{\mathrm{s}}(\mathbf{u} \cdot \boldsymbol{\tau})(\mathbf{v} \cdot \boldsymbol{\tau})+\int_{\Gamma_{\mathrm{g}}} \Lambda_{g} \mathbf{v} \cdot \boldsymbol{\nu}-\mathrm{StCa} \int_{\Omega} \mathbf{f} \cdot \mathbf{v},
$$

where $\boldsymbol{\sigma}:=-p \mathbf{I}+\mathrm{Ca} D(\mathbf{u})$ and $D(\mathbf{u}):=\nabla \mathbf{u}+(\nabla \mathbf{u})^{T}$. Choosing $\mathbf{v}$ to be a smooth test function with compact support in $\Omega$ leads to the Stokes momentum equation:

$$
-\nabla \cdot \sigma=\operatorname{StCa} \mathbf{f}, \quad \text { on } \Omega .
$$

So we are left with

$$
0=\int_{\Gamma_{\mathrm{s}}} \mathbf{v} \cdot \boldsymbol{\sigma} \boldsymbol{\nu}+\int_{\Gamma_{\mathrm{g}}} \mathbf{v} \cdot \boldsymbol{\sigma} \boldsymbol{\nu}+\int_{\Gamma_{\mathrm{s}}} \bar{\beta}_{\mathrm{s}}(\mathbf{u} \cdot \boldsymbol{\tau})(\mathbf{v} \cdot \boldsymbol{\tau})+\int_{\Gamma_{\mathrm{g}}} \Lambda_{g} \mathbf{v} \cdot \boldsymbol{\nu}
$$

and recall that $\boldsymbol{\tau}=\mathbf{e}_{x}$ on $\Gamma_{\mathrm{s}}$. Choose an arbitrary test function $\mathbf{v}=\phi \mathbf{e}_{x}$, with compact $\phi$ on $\Gamma_{\mathrm{s}}$, to get

$$
0=\int_{\Gamma_{\mathrm{s}}} \phi\left[\boldsymbol{\tau} \cdot \boldsymbol{\sigma} \boldsymbol{\nu}+\bar{\beta}_{\mathrm{s}}(\mathbf{u} \cdot \boldsymbol{\tau})\right], \quad \forall \phi, \quad \Rightarrow \quad \boldsymbol{\tau} \cdot \boldsymbol{\sigma} \boldsymbol{\nu}=-\bar{\beta}_{\mathrm{s}} \mathbf{u} \cdot \boldsymbol{\tau}, \quad \text { on } \Gamma_{\mathrm{s}},
$$

which models slip at the solid surface. Hence, we avoid the classic singularity associated with contact line motion. Lastly, choosing $\mathbf{v}$ to be smooth but arbitrary on $\Gamma_{\mathrm{g}}$, we obtain

$$
0=\int_{\Gamma_{\mathrm{g}}}\left[\boldsymbol{\sigma} \boldsymbol{\nu}+\Lambda_{g} \boldsymbol{\nu}\right] \cdot \mathbf{v}, \quad \forall \mathbf{v}, \quad \Rightarrow \quad \boldsymbol{\sigma} \boldsymbol{\nu}=-\Lambda_{g} \boldsymbol{\nu}=-\kappa \boldsymbol{\nu}=\nabla_{\Gamma} \cdot\left[\nabla_{\Gamma} \mathbf{X}\right], \quad \text { on } \Gamma_{\mathrm{g}} .
$$

This formulation has no smoothed Dirac deltas that couple the contact line motion to the interior fluid velocity in an ad-hoc manner. It is also straightforward to include non-linear contact line models by modifying the dissipation functional. 
Remark 2.7. The fluid velocity does not directly appear at the contact line. This seems to be a point of confusion in the fluids community. To better explain, first consider a rigid cylinder rolling on a flat surface with no-slip conditions applied at the surface contact point. In a fixed frame of reference, the contact point is clearly moving. However, the velocity of the cylinder at the contact point vanishes (because of no-slip). Now consider a fluid droplet moving along a surface with approximate no-slip (i.e. large value of $\bar{\beta}_{\mathrm{s}}$ ); the droplet will have a rolling motion as verified by experiments [60]. The same argument shows that the fluid velocity evaluated at the contact point does not correlate with the velocity of the contact point. Despite this, methods are proposed that directly link the fluid velocity to the contact line motion by imposing a "contact line mass balance" relation [4], ([25], Sect. 2.1).

Moreover, imposing a condition involving the bulk fluid velocity at the contact line is mathematically unclear. It is well known that one cannot impose a boundary condition at a point in 2-D (or on a surface of codimension 2) [2]. In particular, the regularity of the fluid velocity evaluated at a point is not well-defined by standard functional analysis $[1,31]$.

\subsection{Formal energy law}

The existence (and uniqueness) of a solution to the system of equations (2.18) and (2.19) is not trivial; see [17] for the case of a non-linear shell interacting with a Navier-Stokes fluid. Moreover, regularity of the solution is completely open. We do not address the well-posedness of the fully continuous formulation in this paper. However, it is possible to obtain a formal energy law for the system.

Proposition 2.8. Assume $\mathbf{f}(t)$ in $L_{\mathrm{loc}}^{2}\left(\mathbb{R}^{2}\right)$ and the initial liquid-gas interface $\Gamma_{\mathrm{g}}(0)$ is $W^{2, \infty}$, with parametrization $\mathbf{X}^{0}$, and $\Gamma_{\mathrm{s}}(0)$ is flat with positive measure. Suppose there exists a solution of the system (2.18) and (2.19) for a.e. $t$ in $[0, T]$ for some $T>0$ such that $\Gamma_{\mathrm{g}}(t)$ is $W^{2, \infty}$, is parameterized by $\mathbf{X}(t)$ with $\mathbf{X} \cdot \mathbf{e}_{y}=0$ on $\partial \Gamma_{\mathrm{g}}(t)$ and $\mathbf{X}(0)=\mathbf{X}^{0}$, and $\Gamma_{\mathrm{S}}(t)$ is flat with positive measure. Moreover, assume $\mathbf{u}(t)$ is in $H^{1}(\Omega(t))$, with $\mathbf{u} \cdot \mathbf{e}_{y}=0$ on $\Gamma_{\mathrm{s}}(t), \dot{\mathbf{X}}(t)$ in $H^{1}\left(\Gamma_{\mathrm{g}}(t)\right), p(t)$ in $L^{2}(\Omega(t)), \Lambda_{g}(t)$ in $L^{2}\left(\Gamma_{\mathrm{g}}(t)\right), \lambda_{L}(t), \lambda_{R}(t)$ in $\mathbb{R}$ for a.e. $t$ in $[0, T]$. Then,

$$
\begin{array}{r}
\int_{0}^{t}\|D(\mathbf{u})\|_{L^{2}(\Omega)}^{2} \mathrm{~d} t+\int_{0}^{t}\left\|\bar{\beta}_{\mathrm{s}} \mathbf{u} \cdot \boldsymbol{\tau}\right\|_{L^{2}\left(\Gamma_{\mathrm{s}}\right)}^{2} \mathrm{~d} t+\left.\int_{0}^{t}\left(\dot{\mathbf{X}} \cdot \mathbf{e}_{x}\right)^{2}\right|_{\mathbf{x}_{\mathrm{cl}}} \mathrm{d} t+\left|\Gamma_{\mathrm{g}}(t)\right| \leq \\
C\left\{\left|\Gamma_{\mathrm{g}}(0)\right|+\int_{0}^{t}\|\mathbf{f}\|_{L^{2}(\Omega)}^{2} \mathrm{~d} t+\left.\int_{0}^{t}\left(\bar{\gamma}_{\mathrm{s}}-\bar{\gamma}_{\mathrm{s}, \mathrm{g}}\right)^{2}\right|_{\mathbf{x}_{\mathrm{cl}}} \mathrm{d} t\right\},
\end{array}
$$

for all $t$ in $[0, T]$, where the constant $C$ only depends on the domain and the non-dimensional parameters in the problem. We use the notation $\left.Z\right|_{\mathbf{x}_{\mathrm{cl}}}:=\left.Z_{L}\right|_{\mathbf{x}_{\mathrm{cl}}^{L}}+\left.Z_{R}\right|_{\mathbf{x}_{\mathrm{cl}}^{R}}$ (with $Z$ being any quantity at $\left.\mathbf{x}_{\mathrm{cl}}\right)$.

Proof. use integration by parts to rewrite the second equation of (2.18) (compare to (2.21)):

$$
\begin{gathered}
\left.\bar{\beta}_{\mathrm{cl}}\left(\dot{\mathbf{X}} \cdot \mathbf{e}_{x}\right) \mathbf{Y} \cdot \mathbf{e}_{x}\right|_{\mathbf{x}_{\mathrm{cl}}^{L}}+\left.\bar{\beta}_{\mathrm{cl}}\left(\dot{\mathbf{X}} \cdot \mathbf{e}_{x}\right) \mathbf{Y} \cdot \mathbf{e}_{x}\right|_{\mathbf{x}_{\mathrm{cl}}^{R}}+\left.\lambda_{L} \mathbf{Y} \cdot \mathbf{e}_{x}\right|_{\mathbf{x}_{\mathrm{cl}}^{L}}+\left.\lambda_{R} \mathbf{Y} \cdot \mathbf{e}_{x}\right|_{\mathbf{x}_{\mathrm{cl}}^{R}} \\
\quad-\int_{\Gamma_{\mathrm{g}}} \Lambda_{g} \mathbf{Y} \cdot \boldsymbol{\nu}+\int_{\Gamma_{\mathrm{g}}} \partial_{s} \mathbf{X} \cdot \partial_{s} \mathbf{Y}=\left.\left(\bar{\gamma}_{\mathrm{s}}-\bar{\gamma}_{\mathrm{s}, \mathrm{g}}\right) \mathbf{Y} \cdot \mathbf{e}_{x}\right|_{\mathbf{x}_{\mathrm{cl}}^{L}}-\left.\left(\bar{\gamma}_{\mathrm{s}}-\bar{\gamma}_{\mathrm{s}, \mathrm{g}}\right) \mathbf{Y} \cdot \mathbf{e}_{x}\right|_{\mathbf{x}_{\mathrm{cl}}^{R}},
\end{gathered}
$$

for all $\mathbf{Y}$ in $H^{1}\left(\Gamma_{\mathrm{g}}(t)\right)$ such that $\mathbf{Y} \cdot \mathbf{e}_{y}=0$ at $\partial \Gamma_{\mathrm{g}}(t)$. In (2.18), choose $\mathbf{v}=\mathbf{u}$ and $q=p$. Next, choose $\mathbf{Y}=\dot{\mathbf{X}}$ in (2.28), and use the following relation [23,40,64]:

$$
\frac{\mathrm{d}}{\mathrm{d} t}\left|\Gamma_{\mathrm{g}}(t)\right|=\frac{\mathrm{d}}{\mathrm{d} t} \int_{\Gamma_{\mathrm{g}}(t)} 1=\int_{\Gamma_{\mathrm{g}}(t)} \partial_{s} \mathbf{X} \cdot \partial_{s} \dot{\mathbf{X}}
$$


Adding the equations together, and choosing $\mu=\Lambda_{g}$, we obtain

$$
\begin{aligned}
\frac{\mathrm{Ca}}{2}\|D(\mathbf{u})\|_{L^{2}(\Omega)}^{2} & +\left\|\bar{\beta}_{\mathrm{s}} \mathbf{u} \cdot \boldsymbol{\tau}\right\|_{L^{2}\left(\Gamma_{\mathrm{s}}\right)}^{2}+\frac{\mathrm{d}}{\mathrm{d} t}\left|\Gamma_{\mathrm{g}}(t)\right|+\left.\bar{\beta}_{\mathrm{cl}}\left(\dot{\mathbf{X}} \cdot \mathbf{e}_{x}\right)^{2}\right|_{\mathbf{x}_{\mathrm{cl}}}+\left.\lambda \dot{\mathbf{X}} \cdot \mathbf{e}_{x}\right|_{\mathbf{x}_{\mathrm{cl}}} \\
& =\operatorname{StCa} \int_{\Omega} \mathbf{f} \cdot \mathbf{u}+\left(\bar{\gamma}_{\mathrm{s}}-\bar{\gamma}_{\mathrm{s}, \mathrm{g}}\right) \dot{\mathbf{X}} \cdot \mathbf{e}_{x}||_{\mathbf{x}_{\mathrm{cl}}^{L}}-\left.\left(\bar{\gamma}_{\mathrm{s}}-\bar{\gamma}_{\mathrm{s}, \mathrm{g}}\right) \dot{\mathbf{X}} \cdot \mathbf{e}_{x}\right|_{\mathbf{x}_{\mathrm{cl}}^{R}},
\end{aligned}
$$

where $\mathbf{x}_{\mathrm{cl}}$ denotes both contact points. Choosing $\xi=0$ in (2.19) yields $\left.\lambda \dot{\mathbf{X}} \cdot \mathbf{e}_{x}\right|_{\mathbf{x}_{\mathrm{cl}}} \geq 0$. Finally, applying Young's inequality "with $\epsilon$ ", using a modified Korn's inequality similar to (3.25), and integrating in time gives the assertion.

\section{WELL-POSEDNESS}

We investigate a time semi-discrete version of the model derived in Section 2.3.7. Specifically, we prove the well-posedness of a single time-step of the semi-discrete weak formulation (see Sect. 3.5), the main results being the normal vector extension (Lem. 3.2) and the inf-sup condition (Thm. 3.12). In addition, we derive a formal energy law (Prop. 3.14) that is analogous to the fully continuous case (Prop. 2.8). A related analysis, concerning Hele-Shaw flow without contact line effects, can be found in [32].

\subsection{Domain assumptions}

We make some basic assumptions on the domain $\Omega$ throughout this section, namely $\partial \Omega=\overline{\Gamma_{\mathrm{g}}} \cup \overline{\Gamma_{\mathrm{s}}}$ is piecewise smooth with corners at the contact points $\mathbf{x}_{\mathrm{cl}}, \Gamma_{\mathrm{s}}$ is flat with positive measure (i.e. the droplet has a non-trivial attachment with the solid surface), and $\Gamma_{\mathrm{g}}$ is $W^{2, \infty}$. The smoothness assumption is reasonable given that surface tension is present.

\subsection{Smoothing the normal vector}

To enable the analysis, we state and prove a result on a "smoothed" version of the unit normal vector $\boldsymbol{\nu}$ of $\partial \Omega$ (recall that $\boldsymbol{\nu}$ is discontinuous at the contact points $\partial \Gamma_{\mathrm{g}}$ ) and extend it into $\Omega$. we need a basic result on extending a function near a corner.

Proposition 3.1 (Corner extension). Consider the wedge geometry in Figure 4 , where $\Gamma_{\mathrm{g}}$ and $\Gamma_{\mathrm{s}}$ are straight lines. Let $f$ be a $W^{1, \infty}$ function defined on $\overline{\Gamma_{\mathrm{g}}} \cup \overline{\Gamma_{\mathrm{s}}}$ such that

$$
f \text { is constant on }\left(\overline{\Gamma_{\mathrm{g}}} \cup \overline{\Gamma_{\mathrm{s}}}\right) \cap B\left(\mathbf{p}, a_{0}\right), \quad \text { for some } a_{0}>0 \text {, }
$$

where $\mathbf{p}=\overline{\Gamma_{\mathrm{g}}} \cap \overline{\Gamma_{\mathrm{s}}}$ and $B\left(\mathbf{x}_{0}, r\right)$ denotes the open ball of radius $r$ centered at $\mathbf{x}_{0}$. Then there exists an extension $f_{E}$, defined on $\bar{\Omega}$, such that $f_{E}$ is $W^{1, \infty}$ and $\left.f_{E}\right|_{\overline{\Gamma_{\mathrm{g}}}} \cup \overline{\Gamma_{\mathrm{s}}}=f$.

Proof. Take the corner point to be the origin in a polar coordinate system $(r, \theta)$. Let $f_{s}=\left.f\right|_{\Gamma_{\mathrm{s}}}$ and $f_{g}=\left.f\right|_{\Gamma_{\mathrm{g}}}$. Thus, $f_{s}$ and $f_{g}$ are functions of $r$ only and $f_{s}(0)=f_{g}(0), f_{s}^{\prime}(0)=f_{g}^{\prime}(0)=0$. Define the extension by

$$
f_{E}(r, \theta)=\left(\frac{\theta}{\theta_{\mathrm{cl}}}\right) f_{g}(r)+\left(1-\frac{\theta}{\theta_{\mathrm{cl}}}\right) f_{s}(r) .
$$

The gradient in polar coordinates is $\nabla=\left(\frac{\partial}{\partial r}, \frac{1}{r} \frac{\partial}{\partial \theta}\right)$. It is straightforward, to show that $\nabla f_{E}$ is continuous. 


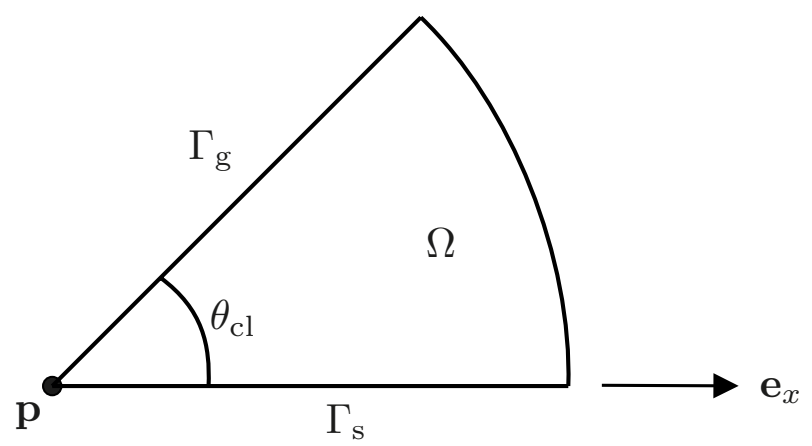

Figure 4. Smooth extension near a corner. A function $f$ is defined on $\overline{\Gamma_{\mathrm{g}}} \cup \overline{\Gamma_{\mathrm{s}}}$ which is extended to all of $\Omega$.

Lemma 3.2. Let $\Omega \subset \mathbb{R}^{2}$ be a bounded domain whose boundary partitions as $\overline{\partial \Omega}=\overline{\Gamma_{\mathrm{g}}} \cup \overline{\Gamma_{\mathrm{s}}}$ with unit outer normal vector $\boldsymbol{\nu}$. Assume the boundary $\partial \Omega$ is $W^{2, \infty}$, except at the contact line $\partial \Gamma_{\mathrm{g}} \equiv \partial \Gamma_{\mathrm{s}} \equiv\left\{\mathbf{x}_{\mathrm{cl}}^{L}, \mathbf{x}_{\mathrm{cl}}^{R}\right\}$ (i.e. two points) where the tangent vector is discontinuous (see Figs. 2 and 3). Furthermore, assume that

$$
\alpha_{0} \leq \boldsymbol{\nu} \cdot\left(-\mathbf{e}_{x}\right) \leq 1 \text {, at } \mathbf{x}_{\mathrm{cl}}^{L}, \quad \alpha_{0} \leq \boldsymbol{\nu} \cdot \mathbf{e}_{x} \leq 1 \text {, at } \mathbf{x}_{\mathrm{cl}}^{R} \text {, where } 0<\alpha_{0}<1,
$$

for some fixed constant $\alpha_{0}$. This means that the contact angle $\cos \theta_{\mathrm{cl}}=\boldsymbol{\nu} \cdot \mathbf{e}_{y}$ is bounded away from 0 and 180 degrees at both points (i.e. strictly convex, non-degenerate corners). Then there exists a $W^{1, \infty}$ vector function $\widetilde{\boldsymbol{\nu}}$ on $\partial \Omega$ such that

$$
\widetilde{\boldsymbol{\nu}} \cdot \boldsymbol{\nu}=1, \text { on } \Gamma_{\mathrm{g}}, \quad \widetilde{\boldsymbol{\nu}} \cdot \mathbf{e}_{y}=0, \text { on } \Gamma_{\mathrm{s}},\left.\quad \widetilde{\boldsymbol{\nu}}\right|_{\partial \Gamma_{\mathrm{g}}}=\left.\frac{\mathbf{e}_{x}}{\mathbf{e}_{x} \cdot \boldsymbol{\nu}}\right|_{\partial \Gamma_{\mathrm{g}}}, \quad\|\widetilde{\boldsymbol{\nu}}\|_{W^{1, \infty}(\partial \Omega)} \leq C,
$$

where $C>0$ is a constant that depends on the curvature of $\Gamma_{\mathrm{g}}$, the length of $\Gamma_{\mathrm{s}}$, and $\alpha_{0}^{-1}$ (note: $\widetilde{\boldsymbol{\nu}}$ is not a unit vector). Furthermore, there is a smooth extension $\widetilde{\boldsymbol{\nu}}_{E}$ of $\widetilde{\boldsymbol{\nu}}$ over $\Omega$ such that

$$
\left.\widetilde{\boldsymbol{\nu}}_{E}\right|_{\partial \Omega}=\widetilde{\boldsymbol{\nu}}, \quad\left\|\widetilde{\boldsymbol{\nu}}_{E}\right\|_{W^{1, \infty}(\Omega)} \leq C .
$$

Proof. We start with the left contact point $\mathbf{x}_{\mathrm{cl}}^{L}$. Let $W_{L}=\Gamma_{\mathrm{g}} \cap B\left(\mathbf{x}_{\mathrm{cl}}^{L}, a_{L}\right)$, where $a_{L}>0$ is such that the unit normal vector of $\Gamma_{\mathrm{g}}$ satisfies (by a Taylor expansion)

$$
\boldsymbol{\nu}=\left.\boldsymbol{\nu}\right|_{\mathbf{x}_{\mathrm{cl}}^{L}}+O\left(\frac{\alpha_{0}}{10}\right), \quad \text { on } W_{L} .
$$

It is straightforward to show that the length of $W_{L}$ is bounded by $\frac{\alpha_{0}}{10} \min \left(\frac{1}{\max _{\Gamma_{\mathrm{g}}}|\kappa|},\left|\Gamma_{\mathrm{g}}\right|\right)$, where $\kappa$ is the curvature of $\Gamma_{\mathrm{g}}$. Similarly, we have $W_{R}=\Gamma_{\mathrm{g}} \cap B\left(\mathbf{x}_{\mathrm{cl}}^{R}, a_{R}\right)$, where $a_{R}>0$ is such that $\boldsymbol{\nu}=\left.\boldsymbol{\nu}\right|_{\mathbf{x}_{\mathrm{cl}}^{R}}+O\left(\alpha_{0} / 10\right)$, on $W_{R}$ and $\left|W_{R}\right|=\frac{\alpha_{0}}{10} \min \left(\frac{1}{\max _{\Gamma \mathrm{g}}|\kappa|},\left|\Gamma_{\mathrm{g}}\right|\right)$. Note that $a_{L}, a_{R}$ are chosen to guarantee that $W_{L} \cap W_{R}=\emptyset$ and $\Gamma_{\mathrm{g}} \backslash\left(W_{L} \cup W_{R}\right)$ has positive measure.

Next, let $\hat{\boldsymbol{\nu}}: \Gamma_{\mathrm{g}} \rightarrow \mathbb{R}^{2}$ be a $W^{1, \infty}$ function defined by a three-piece partition of unity. Let $\chi_{L}: \Gamma_{\mathrm{g}} \rightarrow \mathbb{R}$ be a non-negative $C^{1}$ function such that

$$
\chi_{L}=\left\{\begin{array}{c}
1, \text { on } W_{L} \cap B\left(\mathrm{x}_{\mathrm{cl}}^{L}, a_{L} / 2\right), \\
\text { smooth, on } W_{L}, \\
0, \text { outside } W_{L} .
\end{array}\right.
$$

Define $\chi_{R}$ similarly with respect to $W_{R}$ and set $\chi_{C}=1-\left(\chi_{L}+\chi_{R}\right)$ (i.e. the middle piece); ergo, $\chi_{L}+\chi_{R}+\chi_{C}=1$. Then, $\hat{\boldsymbol{\nu}}$ is given by

$$
\hat{\boldsymbol{\nu}}=-\mathbf{e}_{x} \chi_{L}+\mathbf{e}_{x} \chi_{R}+\boldsymbol{\nu} \chi_{C} .
$$


Finally, define $\widetilde{\boldsymbol{\nu}}: \partial \Omega \rightarrow \mathbb{R}^{2}$ by

$$
\widetilde{\boldsymbol{\nu}}=\frac{\hat{\boldsymbol{\nu}}}{\hat{\boldsymbol{\nu}} \cdot \boldsymbol{\nu}}, \text { on } \Gamma_{\mathrm{g}}
$$

and extend it on $\Gamma_{\mathrm{s}}$ using a similar partition of unity argument, i.e. $\widetilde{\boldsymbol{\nu}}$ is constant near the contact points. Clearly, $\widetilde{\boldsymbol{\nu}} \cdot \boldsymbol{\nu}=1$ on $\Gamma_{\mathrm{g}}$ and $\widetilde{\boldsymbol{\nu}} \cdot \mathbf{e}_{y}=0$ on $\Gamma_{\mathrm{s}}$ because $\widetilde{\boldsymbol{\nu}} \propto \mathbf{e}_{x}$ on $\Gamma_{\mathrm{s}}$. Also, we have that $\hat{\boldsymbol{\nu}} \cdot \boldsymbol{\nu} \geq \alpha_{0} / 2$ uniformly on $\Gamma_{\mathrm{g}}$. Therefore, we get (3.1).

Finally, define $\widetilde{\boldsymbol{\nu}}_{E}$ to be an extension of $\widetilde{\boldsymbol{\nu}}$ to $\Omega$. At the contact points $\mathbf{x}_{\mathrm{cl}}^{L}$ and $\mathbf{x}_{\mathrm{cl}}^{R}$, the extension is done by using Proposition 3.1; note that $\widetilde{\boldsymbol{\nu}}$ is $W^{1, \infty}$ and constant near the contact points. Away from the corner points, one can simply extend the function constant in the normal direction, followed by a mollification in the interior to take care of any "shocks" in the extension.

\subsection{Time-discretization}

For simplicity, we set the constants to unity, except the time-step $\delta t$. To obtain the time-discrete version of (2.18) and (2.19) we apply the following finite difference in time for the interface position:

$$
\dot{\mathbf{X}}^{n+1}(\mathbf{x})=\frac{\mathbf{X}^{n+1}(\mathbf{x})-\mathbf{X}^{n}(\mathbf{x})}{\delta t}, \quad \text { for all } \mathbf{x} \in \Gamma_{\mathrm{g}}^{n}, \quad \text { where } \mathbf{X}^{n} \equiv \operatorname{id}_{\Gamma_{\mathrm{g}}^{n}} \text { (identity map). }
$$

All domains are assumed to be at the current known time-step, i.e. $\Omega \equiv \Omega^{n}=\Omega\left(t_{n}\right)$, etc., but all solution variables are considered at the next time-step (i.e. implicit):

$$
\mathbf{u} \equiv \mathbf{u}^{n+1}, \quad \mathbf{W} \equiv \mathbf{X}^{n+1}, \quad \operatorname{id}_{\Gamma_{\mathrm{g}}} \equiv \mathbf{X}^{n}, \quad p \equiv p^{n+1}, \quad \Lambda_{g} \equiv \Lambda_{g}^{n+1}, \quad \lambda_{L} \equiv \lambda_{L}^{n+1}, \quad \lambda_{R} \equiv \lambda_{R}^{n+1} .
$$

We replace $\mathbf{X}^{n+1}$ by $\mathbf{W}$ to emphasize that it is a solution variable in the formulation. Note that $\mathbf{X}^{n+1}$ parameterizes the domain $\Gamma_{\mathrm{g}}^{n+1}$ at the next time step. So inserting (3.3) into (2.18) and (2.19), using the integration by parts result (2.28) and ignoring the physical constants, we obtain the time semi-discrete weak formulation:

$$
\begin{aligned}
& \frac{1}{2} \int_{\Omega} D(\mathbf{u}): D(\mathbf{v})-\int_{\Omega} p \nabla \cdot \mathbf{v}+\int_{\Gamma_{\mathrm{s}}}(\mathbf{u} \cdot \boldsymbol{\tau})(\mathbf{v} \cdot \boldsymbol{\tau})+\int_{\Gamma_{\mathrm{g}}} \Lambda_{g} \mathbf{v} \cdot \boldsymbol{\nu}=\int_{\Omega} \mathbf{f} \cdot \mathbf{v}, \quad \text { for all admissible } \mathbf{v}, \\
& \left.\frac{1}{\delta t}\left(\mathbf{W} \cdot \mathbf{e}_{x}\right) \mathbf{Y} \cdot \mathbf{e}_{x}\right|_{\mathbf{x}_{\mathrm{cl}}^{L}}+\left.\frac{1}{\delta t}\left(\mathbf{W} \cdot \mathbf{e}_{x}\right) \mathbf{Y} \cdot \mathbf{e}_{x}\right|_{\mathbf{x}_{\mathrm{cl}}^{R}}+\left.\lambda_{L} \mathbf{Y} \cdot \mathbf{e}_{x}\right|_{\mathbf{x}_{\mathrm{cl}}^{L}}+\left.\lambda_{R} \mathbf{Y} \cdot \mathbf{e}_{x}\right|_{\mathbf{x}_{\mathrm{cl}}^{R}} \\
& \quad-\int_{\Gamma_{\mathrm{g}}} \Lambda_{g} \mathbf{Y} \cdot \boldsymbol{\nu}+\int_{\Gamma_{\mathrm{g}}} \partial_{s} \mathbf{W} \cdot \partial_{s} \mathbf{Y}=\left.\frac{1}{\delta t}\left(\mathrm{id}_{\Gamma_{\mathrm{g}}} \cdot \mathbf{e}_{x}\right) \mathbf{Y} \cdot \mathbf{e}_{x}\right|_{\mathbf{x}_{\mathrm{cl}}^{L}}+\left.\frac{1}{\delta t}\left(\mathrm{id}_{\Gamma_{\mathrm{g}}} \cdot \mathbf{e}_{x}\right) \mathbf{Y} \cdot \mathbf{e}_{x}\right|_{\mathbf{x}_{\mathrm{cl}}^{R}} \\
& \quad+\left.\left(\gamma_{\mathrm{s}}-\gamma_{\mathrm{s}, \mathrm{g}}\right) \mathbf{Y} \cdot \mathbf{e}_{x}\right|_{\mathbf{x}_{\mathrm{cl}}^{L}}-\left.\left(\gamma_{\mathrm{s}}-\gamma_{\mathrm{s}, \mathrm{g}}\right) \mathbf{Y} \cdot \mathbf{e}_{x}\right|_{\mathbf{x}_{\mathrm{cl}}^{R}}, \quad \text { for all admissible } \mathbf{Y}, \\
& \quad-\int_{\Omega} q \nabla \cdot \mathbf{u}=0, \quad \text { for all admissible } q, \\
& \quad \int_{\Gamma_{\mathrm{g}}} \mu(\mathbf{u} \cdot \boldsymbol{\nu})-\frac{1}{\delta t} \int_{\Gamma_{\mathrm{g}}} \mu(\mathbf{W} \cdot \boldsymbol{\nu})=-\frac{1}{\delta t} \int_{\Gamma_{\mathrm{g}}} \mu\left(\mathrm{id}_{\Gamma_{\mathrm{g}}} \cdot \boldsymbol{\nu}\right), \quad \text { for all admissible } \mu, \\
& \left.\frac{1}{\delta t}\left(\xi-\lambda_{J}\right)\left(\mathbf{W} \cdot \mathbf{e}_{x}\right)\right|_{\mathbf{x}_{\mathrm{cl}}^{J}} \leq\left.\frac{1}{\delta t}\left(\xi-\lambda_{J}\right)\left(\mathrm{id}_{\Gamma_{\mathrm{g}}} \cdot \mathbf{e}_{x}\right)\right|_{\mathbf{x}_{\mathrm{c} 1}^{J}}, \quad \text { for all } \xi \text { in }[-1,1] \subset \mathbb{R}, \quad \text { for } J=L, R .
\end{aligned}
$$

Section 3.4 describes the proper function space setting for (3.4), followed by the abstract mixed formulation in Section 3.5. Most of our analysis in the following sections is limited to a single time-step, but we do obtain a formal energy law in Proposition 3.14 which implies that the time-dependent formulation is stable.

\subsection{Function spaces}

All spaces are written with respect to the current domain, $\Omega^{n} \equiv \Omega$, etc. The velocity space is

$$
\mathbb{V}=\left\{\mathbf{v} \in H^{1}(\Omega): \mathbf{v} \cdot \mathbf{e}_{y}=0, \text { on } \Gamma_{\mathrm{s}}\right\},
$$


with norm denoted $\|\cdot\|_{H^{1}(\Omega)}$, and the pressure space is

$$
\mathbb{Q}=L^{2}(\Omega),
$$

with norm denoted $\|\cdot\|_{L^{2}(\Omega)}$. The space for the position $\mathbf{W} \equiv \mathbf{X}^{n+1}$ is

$$
\mathbb{Y}=\left\{\mathbf{Y} \in H^{1}\left(\Gamma_{\mathrm{g}}\right): \mathbf{Y} \cdot \mathbf{e}_{y}=0, \text { at } \partial \Gamma_{\mathrm{g}}\right\},
$$

with norm denoted $\|\cdot\|_{H^{1}\left(\Gamma_{\mathrm{g}}\right)}$; this space was chosen because of the $H^{1}$ inner product $\int_{\Gamma_{\mathrm{g}}} \partial_{S} \mathbf{X} \cdot \partial_{S} \mathbf{Y}$ appearing in (2.18) (see also (2.28)). The space for the pinning variables $\lambda_{L}, \lambda_{R}$ is $\mathbb{R}$, but confined to a convex set $\mathcal{K}:=[-1,1] \subset \mathbb{R}$.

Next, we have $\mathbf{v}$ in $H^{1 / 2}\left(\Gamma_{\mathrm{g}}\right)$ for all $\mathbf{v}$ in $\mathbb{V}$. Note that $\mathbf{v} \cdot \mathbf{e}_{y}$ is in $H_{00}^{1 / 2}\left(\Gamma_{\mathrm{g}}\right)$ and $\mathbf{v} \cdot \mathbf{e}_{x}$ is in $H^{1 / 2}\left(\Gamma_{\mathrm{g}}\right)$ (provided the contact angle is bounded away from 0 and 180 degrees). Since the unit vector $\nu$ is $W^{1, \infty}$ on $\Gamma_{\mathrm{g}}$, the product $\mathbf{v} \cdot \boldsymbol{\nu}$ is also in $H^{1 / 2}\left(\Gamma_{\mathrm{g}}\right)[1,8,44]$. Thus, by the fourth equation in (3.4), we have that $\Lambda_{g}$ is in

$$
\mathbb{M}=\left(H^{1 / 2}\left(\Gamma_{\mathrm{g}}\right)\right)^{*} .
$$

The next proposition provides a convenient way to define the $\mathbb{M}$ norm and helps facilitate proving the "inf-sup" condition.

Proposition 3.3. For any $\phi$ in $H^{1 / 2}\left(\Gamma_{\mathrm{g}}\right)$, there exists $a \mathbf{v}$ in $\mathbb{V}$ such that $\phi=\mathbf{v} \cdot \boldsymbol{\nu}$ on $\Gamma_{\mathrm{g}}$, where $\boldsymbol{\nu}$ is the unit outer pointing normal vector on $\partial \Omega$. Therefore, the range of the normal trace operator on $\Gamma_{\mathrm{g}}$ over all $\mathbf{v}$ in $\mathbb{V}$ is equal to $H^{1 / 2}\left(\Gamma_{\mathrm{g}}\right)$.

Proof. For any $\phi$ in $H^{1 / 2}\left(\Gamma_{\mathrm{g}}\right)$, there is a $\tilde{\phi}$ in $H^{1}(\Omega)$ such that $\phi=\left.\tilde{\phi}\right|_{\Gamma_{\mathrm{g}}}$. Using Lemma 3.2, define $\mathbf{v}:=\tilde{\phi} \widetilde{\boldsymbol{\nu}}_{E}$. Since $\widetilde{\boldsymbol{\nu}}_{E}$ is in $W^{1, \infty}(\Omega)$, we have that $\mathbf{v}$ is in $H^{1}(\Omega)$ by standard estimates and Sobolev embedding. In fact, $\mathbf{v}$ is in $\mathbb{V}$ because $\mathbf{v} \cdot \mathbf{e}_{y}=0$ on $\Gamma_{\mathrm{s}}$. Moreover, $\left.\mathbf{v} \cdot \boldsymbol{\nu}\right|_{\Gamma_{\mathrm{g}}}=\left(\left.\tilde{\phi}\right|_{\Gamma_{\mathrm{g}}}\right)\left(\left.\widetilde{\boldsymbol{\nu}}_{E} \cdot \boldsymbol{\nu}\right|_{\Gamma_{\mathrm{g}}}\right)=\phi$. Hence, $H^{1 / 2}\left(\Gamma_{\mathrm{g}}\right)$ is contained in the range of the normal trace operator, on $\Gamma_{\mathrm{g}}$, on $\mathbb{V}$ (and vice versa). So we get equality of the spaces.

The integral $\int_{\Gamma_{\mathrm{g}}} \mu \mathbf{v} \cdot \boldsymbol{\nu}$ only makes sense when the functions are suitably regular. Thus, we replace it by the duality pair $\langle\mu, \mathbf{v} \cdot \boldsymbol{\nu}\rangle_{\mathbb{M}}$ for all $\mu$ in $\mathbb{M}$ and $\mathbf{v}$ in $\mathbb{V}$, such that

$$
\langle\mu, \mathbf{v} \cdot \boldsymbol{\nu}\rangle_{\mathbb{M}}=\int_{\Gamma_{\mathrm{g}}} \mu \mathbf{v} \cdot \boldsymbol{\nu}, \quad \text { if } \mu \text { is in } L^{2}\left(\Gamma_{\mathrm{g}}\right) .
$$

By Proposition 3.3 , the $\mathbb{M}$ norm can be written as

$$
\|\mu\|_{\mathbb{M}}=\sup _{\mathbf{v} \in \mathbb{V}} \frac{\langle\mu, \mathbf{v} \cdot \boldsymbol{\nu}\rangle_{\mathbb{M}}}{\|\mathbf{v}\|_{H^{1}(\Omega)}}
$$

which yields the following as an immediate consequence.

Proposition 3.4. Given any $\mu$ in $\mathbb{M}$, there exists $a \mathbf{v}$ in $\mathbb{V}$ such that

$$
\langle\mu, \mathbf{v} \cdot \boldsymbol{\nu}\rangle_{\mathbb{M}}=\|\mu\|_{\mathbb{M}}, \quad\|\mathbf{v}\|_{H^{1}(\Omega)}=1 .
$$

\subsection{Semi-discrete weak formulation}

\subsubsection{Bilinear forms}

Define the primal form

$$
\begin{aligned}
a((\mathbf{u}, \mathbf{W}),(\mathbf{v}, \mathbf{Y}))= & \int_{\Omega} D(\mathbf{u}): \nabla \mathbf{v}+\int_{\Gamma_{\mathrm{s}}}(\mathbf{u} \cdot \boldsymbol{\tau})(\mathbf{v} \cdot \boldsymbol{\tau})+\frac{1}{\delta t} \int_{\Gamma_{\mathrm{g}}} \partial_{s} \mathbf{W} \cdot \partial_{s} \mathbf{Y} \\
& +\left.\frac{1}{\delta t^{2}}\left(\mathbf{W} \cdot \mathbf{e}_{x}\right)\left(\mathbf{Y} \cdot \mathbf{e}_{x}\right)\right|_{\mathbf{x}_{\mathrm{cl}}^{L}}+\left.\frac{1}{\delta t^{2}}\left(\mathbf{W} \cdot \mathbf{e}_{x}\right)\left(\mathbf{Y} \cdot \mathbf{e}_{x}\right)\right|_{\mathbf{x}_{\mathrm{c} 1}^{R}},
\end{aligned}
$$


next the constraint form

$$
\begin{aligned}
b\left((\mathbf{v}, \mathbf{Y}),\left(q, \mu, \xi_{L}, \xi_{R}\right)\right)= & -\int_{\Omega} q \nabla \cdot \mathbf{v}+\langle\mu, \mathbf{v} \cdot \boldsymbol{\nu}\rangle_{\mathbb{M}}-\frac{1}{\delta t}\langle\mu, \mathbf{Y} \cdot \boldsymbol{\nu}\rangle_{\mathbb{M}} \\
& +\left.\frac{1}{\delta t} \xi_{L}\left(\mathbf{Y} \cdot \mathbf{e}_{x}\right)\right|_{\mathbf{x}_{\mathrm{cl}}^{L}}+\left.\frac{1}{\delta t} \xi_{R}\left(\mathbf{Y} \cdot \mathbf{e}_{x}\right)\right|_{\mathbf{x}_{\mathrm{cl}}^{R}},
\end{aligned}
$$

and the linear forms

$$
\begin{aligned}
F((\mathbf{v}, \mathbf{Y}))= & \int_{\Omega} \mathbf{f} \cdot \mathbf{v}+\left.\frac{1}{\delta t}\left(\gamma_{\mathrm{s}}-\gamma_{\mathrm{s}, \mathrm{g}}\right)\left(\mathbf{Y} \cdot \mathbf{e}_{x}\right)\right|_{\mathbf{x}_{\mathrm{cl}}^{L}}-\left.\frac{1}{\delta t}\left(\gamma_{\mathrm{s}}-\gamma_{\mathrm{s}, \mathrm{g}}\right)\left(\mathbf{Y} \cdot \mathbf{e}_{x}\right)\right|_{\mathbf{x}_{\mathrm{cl}}^{R}} \\
& +\left.\frac{1}{\delta t^{2}}\left(\operatorname{id}_{\Gamma_{\mathrm{g}}} \cdot \mathbf{e}_{x}\right)\left(\mathbf{Y} \cdot \mathbf{e}_{x}\right)\right|_{\mathbf{x}_{\mathrm{cl}}^{L}}+\left.\frac{1}{\delta t^{2}}\left(\operatorname{id}_{\Gamma_{\mathrm{g}}} \cdot \mathbf{e}_{x}\right)\left(\mathbf{Y} \cdot \mathbf{e}_{x}\right)\right|_{\mathbf{x}_{\mathrm{cl}}^{R}}, \\
G\left(\left(q, \mu, \xi_{L}, \xi_{R}\right)\right)= & -\frac{1}{\delta t}\left\langle\mu, \operatorname{id}_{\Gamma_{\mathrm{g}}} \cdot \boldsymbol{\nu}\right\rangle_{\mathbb{M}}+\left.\frac{1}{\delta t} \xi_{L}\left(\operatorname{id}_{\Gamma_{\mathrm{g}}} \cdot \mathbf{e}_{x}\right)\right|_{\mathbf{x}_{\mathrm{cl}}^{L}}+\left.\frac{1}{\delta t} \xi_{R}\left(\operatorname{id}_{\Gamma_{\mathrm{g}}} \cdot \mathbf{e}_{x}\right)\right|_{\mathbf{x}_{\mathrm{cl}}^{R}},
\end{aligned}
$$

where $\operatorname{id}_{\Gamma_{\mathrm{g}}}$ is the identity map on $\Gamma_{\mathrm{g}}$. The extra factors of $\frac{1}{\delta t}$ occur by multiplying the second equation in (3.4) by $\frac{1}{\delta t}$. This is done to ensure symmetry of the saddle-point system (3.20).

\subsubsection{Mixed formulation}

Define the primal and multiplier spaces

$$
\mathbb{Z}=\mathbb{V} \times \mathbb{Y}, \quad \mathbb{T}=\mathbb{Q} \times \mathbb{M} \times \mathcal{K} \times \mathcal{K},
$$

along with the primal norm

$$
\|(\mathbf{v}, \mathbf{Y})\|_{\mathbb{Z}^{2}}^{2}=\|\mathbf{v}\|_{H^{1}(\Omega)}^{2}+\|\mathbf{Y}\|_{H^{1}\left(\Gamma_{\mathrm{g}}\right)}^{2},
$$

and multiplier norm: $\left\|\left|\left(q, \mu, \xi_{L}, \xi_{R}\right)\right|\right\|_{\mathbb{T}}^{2}=\|q\|_{L^{2}(\Omega)}^{2}+\|\mu\|_{\mathbb{M}}^{2}+\left|\xi_{L}\right|^{2}+\left|\xi_{R}\right|^{2}$. Let $H^{-1}\left(\Gamma_{\mathrm{g}}\right):=\left(H_{0}^{1}\left(\Gamma_{\mathrm{g}}\right)\right)^{*}$ with norm given by

$$
\|\eta\|_{H^{-1}\left(\Gamma_{\mathrm{g}}\right)}=\sup _{y \in H_{0}^{1}\left(\Gamma_{\mathrm{g}}\right)} \frac{\langle\eta, y\rangle_{*}}{\|y\|_{H^{1}\left(\Gamma_{\mathrm{g}}\right)}},
$$

where $\langle\cdot, \cdot\rangle_{*}$ denotes the duality pairing between $H^{-1}\left(\Gamma_{\mathrm{g}}\right)$ and $H_{0}^{1}\left(\Gamma_{\mathrm{g}}\right)$. For the analysis, we will use an alternative norm for the multipliers, namely

$$
\left\|\left(q, \mu, \xi_{L}, \xi_{R}\right)\right\|_{\mathbb{T}}^{2}=\|\tilde{q}\|_{L^{2}(\Omega)}^{2}+\left\|\mu-q_{0}\right\|_{\mathbb{M}}^{2}+\|\mu\|_{H^{-1}\left(\Gamma_{\mathrm{g}}\right)}^{2}+\left|\xi_{L}\right|^{2}+\left|\xi_{R}\right|^{2},
$$

where $q_{0}=\frac{1}{|\Omega|} \int_{\Omega} q$ and $\tilde{q}=q-q_{0}$. Of course, both multiplier space norms are equivalent, meaning there exists a constant $C>0$ (depending only on $\Omega$ ) such that

$$
\frac{1}{C}\left\|\left|\left(q, \mu, \xi_{L}, \xi_{R}\right)\left\|_{\mathbb{T}} \leq\right\|\left(q, \mu, \xi_{L}, \xi_{R}\right)\left\|_{\mathbb{T}} \leq C \mid\right\|\left(q, \mu, \xi_{L}, \xi_{R}\right) \|_{\mathbb{T}} .\right.\right.
$$

Therefore, at each time-step, the mixed formulation is: find $(\mathbf{u}, \mathbf{W})$ in $\mathbb{Z}$ and $\left(p, \Lambda_{g}, \lambda_{L}, \lambda_{R}\right)$ in $\mathbb{T}$ such that

$$
\begin{aligned}
a((\mathbf{u}, \mathbf{W}),(\mathbf{v}, \mathbf{Y}))+b\left((\mathbf{v}, \mathbf{Y}),\left(p, \Lambda_{g}, \lambda_{L}, \lambda_{R}\right)\right) & =F((\mathbf{v}, \mathbf{Y})), \\
b\left((\mathbf{u}, \mathbf{W}),\left(q, \mu, \xi_{L}-\lambda_{L}, \xi_{R}-\lambda_{R}\right)\right) & \leq G\left(\left(q, \mu, \xi_{L}-\lambda_{L}, \xi_{R}-\lambda_{R}\right)\right),
\end{aligned}
$$

for all $(\mathbf{v}, \mathbf{Y})$ in $\mathbb{Z}$ and $\left(q, \mu, \xi_{L}, \xi_{R}\right)$ in $\mathbb{T}$. The system (3.20) is the abstract form of (3.4).

Remark 3.5 (Time-dependent simulation). The full dynamic method is as follows. Given an initial domain $\Omega^{n}$, compute the solution of (3.20). Update the domain boundary with $\mathbf{X}^{n+1}:=\mathbf{W}^{n+1}$; this defines a new domain $\Omega^{n+1}$ at the next time step. This procedure repeats as long as needed. 


\subsection{Semi-discrete analysis}

\subsubsection{Continuity}

Lemma 3.6 (Continuity). Let $\Omega$ be an open bounded set in $\mathbb{R}^{d}$ with Lipschitz boundary $\partial \Omega=\overline{\Gamma_{\mathrm{g}}} \cup \overline{\Gamma_{\mathrm{s}}}$, such that $\Gamma_{\mathrm{g}}, \Gamma_{\mathrm{s}}$ are $W^{2, \infty}$. Then there are positive constants $C_{a}, C_{b}, C_{F}, C_{G}$ that depend on $\Omega$ and $\delta t^{-1}$ such that

$$
\begin{aligned}
a((\mathbf{u}, \mathbf{W}),(\mathbf{v}, \mathbf{Y})) & \leq C_{a}\|(\mathbf{u}, \mathbf{W})\|_{\mathbb{Z}}\|(\mathbf{v}, \mathbf{Y})\|_{\mathbb{Z}}, \\
b\left((\mathbf{u}, \mathbf{W}),\left(q, \mu, \xi_{L}, \xi_{R}\right)\right) & \leq C_{b}\|(\mathbf{u}, \mathbf{W})\|_{\mathbb{Z}}\left\|\left(q, \mu, \xi_{L}, \xi_{R}\right)\right\|_{\mathbb{T}}, \\
F((\mathbf{v}, \mathbf{Y})) & \leq C_{F}\|(\mathbf{v}, \mathbf{Y})\|_{\mathbb{Z}} \\
G\left(\left(q, \mu, \xi_{L}, \xi_{R}\right)\right) & \leq C_{G}\left\|\left(q, \mu, \xi_{L}, \xi_{R}\right)\right\|_{\mathbb{T}},
\end{aligned}
$$

for all $(\mathbf{u}, \mathbf{W}),(\mathbf{v}, \mathbf{Y})$ in $\mathbb{Z}$ and $\left(q, \mu, \xi_{L}, \xi_{R}\right)$ in $\mathbb{T}$.

Proof. The bound (3.21) follows by Cauchy-Schwarz and standard trace estimates. Likewise for (3.22), one applies Cauchy-Schwarz and uses (3.19). The same goes for (3.23).

\subsubsection{Coercivity}

We first state some standard results.

Lemma 3.7 (Korn's Inequality). Let $\Omega$ be an open bounded set in $\mathbb{R}^{d}$ with Lipschitz boundary. Then there exists a number $A_{0}=A_{0}(\Omega)>0$ such that

$$
\int_{\Omega} D(\mathbf{v}): D(\mathbf{v})+\|\mathbf{v}\|_{L^{2}(\Omega)}^{2} \geq A_{0}\|\mathbf{v}\|_{H^{1}(\Omega)}^{2}, \text { for all } \mathbf{v} \text { in } H^{1}(\Omega) .
$$

Proof. See $[19,26]$.

A standard argument leads to the following inequality (useful for our problem).

Lemma 3.8 (Modified Korn's Inequality). Let $\Omega$ be an open bounded set in $\mathbb{R}^{2}$ with Lipschitz boundary $\partial \Omega=$ $\overline{\Gamma_{\mathrm{g}}} \cup \overline{\Gamma_{\mathrm{s}}}$, such that $\Gamma_{\mathrm{g}}, \Gamma_{\mathrm{s}}$ are $W^{2, \infty}$. Suppose $\Gamma_{\mathrm{s}}$ has positive measure (length). Then

$$
\int_{\Gamma_{\mathrm{s}}}(\mathbf{v} \cdot \boldsymbol{\tau})^{2}+\int_{\Omega} D(\mathbf{v}): D(\mathbf{v}) \geq A_{1}\|\mathbf{v}\|_{H^{1}(\Omega)}^{2}, \quad \text { for all } \mathbf{v} \text { in } \mathbb{V},
$$

where $A_{1}=A_{1}\left(\Omega, \Gamma_{\mathrm{s}}\right)>0$ and $\boldsymbol{\tau}$ is the unit tangent vector of $\Gamma_{\mathrm{s}}$.

Lemma 3.9 (Poincaré-Type Inequality). Let $\Gamma_{\mathrm{g}} \subset \mathbb{R}^{2}$ be a $W^{2, \infty}$ curve with boundary $\partial \Gamma_{\mathrm{g}}=\left\{\mathbf{x}_{\mathrm{cl}}^{L}, \mathbf{x}_{\mathrm{cl}}^{R}\right\}$. Then there exists a number $A_{2}=A_{2}\left(\Gamma_{\mathrm{g}}\right)>0$ such that

$$
\left\|\partial_{s} \mathbf{Y}\right\|_{L^{2}\left(\Gamma_{\mathrm{g}}\right)}^{2}+\left.\left(\mathbf{Y} \cdot \mathbf{e}_{x}\right)^{2}\right|_{\mathbf{x}_{\mathrm{cl}}^{L}}+\left.\left(\mathbf{Y} \cdot \mathbf{e}_{x}\right)^{2}\right|_{\mathbf{x}_{\mathrm{cl}}^{R}} \geq A_{2}\|\mathbf{Y}\|_{L^{2}\left(\Gamma_{\mathrm{g}}\right)}^{2}, \quad \text { for all } \mathbf{Y} \text { in } \mathbb{Y}
$$

Proof. Start with the $L^{2}\left(\Gamma_{\mathrm{g}}\right)$ norm and integrate by parts:

$$
\begin{aligned}
\|\mathbf{Y}\|_{L^{2}\left(\Gamma_{\mathrm{g}}\right)}^{2} & =\int_{\Gamma_{\mathrm{g}}} \mathbf{Y} \cdot \mathbf{Y}=\int_{\Gamma_{\mathrm{g}}} \partial_{s}\left(s-\frac{\left|\Gamma_{\mathrm{g}}\right|}{2}\right) \mathbf{Y} \cdot \mathbf{Y} \\
& =-\int_{\Gamma_{\mathrm{g}}}\left(s-\frac{\left|\Gamma_{\mathrm{g}}\right|}{2}\right) 2\left(\partial_{s} \mathbf{Y}\right) \cdot \mathbf{Y}+\left.\frac{\left|\Gamma_{\mathrm{g}}\right|}{2}\left(\mathbf{Y} \cdot \mathbf{e}_{x}\right)^{2}\right|_{\mathbf{x}_{\mathrm{cl}}^{L}}-\left.\frac{\left|\Gamma_{\mathrm{g}}\right|}{2}\left(\mathbf{Y} \cdot \mathbf{e}_{x}\right)^{2}\right|_{\mathbf{x}_{\mathrm{cl}}^{R}}
\end{aligned}
$$


because $\mathbf{Y} \cdot \mathbf{e}_{y}=0$ on $\partial \Gamma_{\mathrm{g}}$; note that $s=0$ at $\mathbf{x}_{\mathrm{cl}}^{R}$ and $s=\left|\Gamma_{\mathrm{g}}\right|$ at $\mathbf{x}_{\mathrm{cl}}^{L}$. Bounding (3.27) and applying CauchySchwarz gives

$$
\begin{aligned}
\|\mathbf{Y}\|_{L^{2}\left(\Gamma_{\mathrm{g}}\right)}^{2} & \leq\left|\Gamma_{\mathrm{g}}\right|\left\|\partial_{s} \mathbf{Y}\right\|_{L^{2}\left(\Gamma_{\mathrm{g}}\right)}\|\mathbf{Y}\|_{L^{2}\left(\Gamma_{\mathrm{g}}\right)}+\left.\frac{\left|\Gamma_{\mathrm{g}}\right|}{2}\left(\mathbf{Y} \cdot \mathbf{e}_{x}\right)^{2}\right|_{\mathbf{x}_{\mathrm{cl}}^{L}}+\left.\frac{\left|\Gamma_{\mathrm{g}}\right|}{2}\left(\mathbf{Y} \cdot \mathbf{e}_{x}\right)^{2}\right|_{\mathbf{x}_{\mathrm{cl}}^{R}} \\
& \leq \frac{\left|\Gamma_{\mathrm{g}}\right|^{2}}{2}\left\|\partial_{s} \mathbf{Y}\right\|_{L^{2}\left(\Gamma_{\mathrm{g}}\right)}^{2}+\frac{1}{2}\|\mathbf{Y}\|_{L^{2}\left(\Gamma_{\mathrm{g}}\right)}^{2}+\left.\frac{\left|\Gamma_{\mathrm{g}}\right|}{2}\left(\mathbf{Y} \cdot \mathbf{e}_{x}\right)^{2}\right|_{\mathbf{x}_{\mathrm{cl}}^{L}}+\left.\frac{\left|\Gamma_{\mathrm{g}}\right|}{2}\left(\mathbf{Y} \cdot \mathbf{e}_{x}\right)^{2}\right|_{\mathbf{x}_{\mathrm{c} 1}^{R}} .
\end{aligned}
$$

This leads to

$$
\|\mathbf{Y}\|_{L^{2}\left(\Gamma_{\mathrm{g}}\right)}^{2} \leq\left|\Gamma_{\mathrm{g}}\right|\left(\left|\Gamma_{\mathrm{g}}\right|\left\|\partial_{s} \mathbf{Y}\right\|_{L^{2}\left(\Gamma_{\mathrm{g}}\right)}^{2}+\left.\left(\mathbf{Y} \cdot \mathbf{e}_{x}\right)^{2}\right|_{\mathbf{x}_{\mathrm{cl}}^{L}}+\left.\left(\mathbf{Y} \cdot \mathbf{e}_{x}\right)^{2}\right|_{\mathbf{x}_{\mathrm{cl}}^{R}}\right),
$$

which proves the assertion.

Putting everything together gives the following coercivity result.

Theorem 3.10. Assume the hypothesis of Lemmas 3.8 and 3.9. For all $\mathbf{v}$ in $\mathbb{V}$ and all $\mathbf{Y}$ in $\mathbb{Y}$, we have

$$
a((\mathbf{v}, \mathbf{Y}),(\mathbf{v}, \mathbf{Y})) \geq A_{3}\left(\|\mathbf{v}\|_{H^{1}(\Omega)}^{2}+\|\mathbf{Y}\|_{H^{1}\left(\Gamma_{\mathrm{g}}\right)}^{2}\right)=A_{3}\|(\mathbf{v}, \mathbf{Y})\|_{\mathbb{Z}}^{2},
$$

for some positive constant $A_{3}$ that depends on $\Omega$ and $\delta t^{-1}$ (for $\left.\delta t \leq 1\right)$.

Proof. Starting with (3.12), we get by Lemmas 3.8 and 3.9

$$
\begin{aligned}
a((\mathbf{v}, \mathbf{Y}),(\mathbf{v}, \mathbf{Y})) & =\frac{1}{2}\|D(\mathbf{v})\|_{L^{2}(\Omega)}^{2}+\|\mathbf{v} \cdot \boldsymbol{\tau}\|_{L^{2}\left(\Gamma_{\mathrm{s}}\right)}^{2}+\frac{1}{\delta t}\left\|\partial_{s} \mathbf{Y}\right\|_{L^{2}\left(\Gamma_{\mathrm{g}}\right)}^{2}+\frac{1}{\delta t^{2}}\left[\left.\left(\mathbf{Y} \cdot \mathbf{e}_{x}\right)^{2}\right|_{\mathbf{x}_{\mathrm{cl}}^{L}}+\left.\left(\mathbf{Y} \cdot \mathbf{e}_{x}\right)^{2}\right|_{\mathbf{x}_{\mathrm{cl}}^{R}}\right] \\
& \geq \frac{1}{2}\left\{A_{1}\|\mathbf{v}\|_{H^{1}(\Omega)}^{2}+\frac{\min \left(1, A_{2}\right)}{\delta t}\|\mathbf{Y}\|_{H^{1}\left(\Gamma_{\mathrm{g}}\right)}^{2}\right\},
\end{aligned}
$$

which gives the assertion.

\subsubsection{Inf-Sup}

The next lemma constructs "matching" functions on $\partial \Omega$ for use in the duality pairing $\langle\cdot, \cdot\rangle_{\mathbb{M}}$.

Lemma 3.11. Let $\mu$ be in $\mathbb{M}$ and $\xi_{L}, \xi_{R}$ in $\mathbb{R}$. Then we have the following results.

1. There exists a $\mathbf{Y}_{0}$ in $\mathbb{Y}$ such that

$$
\mathbf{Y}_{0}=\mathbf{0}, \text { on } \partial \Gamma_{\mathrm{g}}, \quad\left\langle\mu, \mathbf{Y}_{0} \cdot \boldsymbol{\nu}\right\rangle_{\mathbb{M}}=\|\mu\|_{H^{-1}\left(\Gamma_{\mathrm{g}}\right)}, \quad\left\|\mathbf{Y}_{0}\right\|_{H^{1}\left(\Gamma_{\mathrm{g}}\right)} \leq C_{0},
$$

where $C_{0}$ depends on $\widetilde{\boldsymbol{\nu}}$ in Lemma 3.2 and the geometry of $\Gamma_{\mathrm{g}}$.

2. There exists a $\mathbf{Y}_{1}$ in $\mathbb{Y}$ such that

$$
\begin{aligned}
\left.\mathbf{Y}_{1}\right|_{\mathbf{x}_{\mathrm{cl}}^{L}} & =-\operatorname{sgn}\left(\xi_{L}\right) \frac{\mathbf{e}_{x}}{\mathbf{e}_{x} \cdot \boldsymbol{\nu}},\left.\quad \mathbf{Y}_{1}\right|_{\mathbf{x}_{\mathrm{cl}}^{R}}=\operatorname{sgn}\left(\xi_{R}\right) \frac{\mathbf{e}_{x}}{\mathbf{e}_{x} \cdot \boldsymbol{\nu}}, \quad\left\langle\mu, \mathbf{Y}_{1} \cdot \boldsymbol{\nu}\right\rangle_{\mathbb{M}}=0, \\
\frac{\left|\xi_{L}\right|}{\alpha_{0}} & \geq\left.\xi_{L}\left(\mathbf{Y}_{1} \cdot \mathbf{e}_{x}\right)\right|_{\mathbf{x}_{\mathrm{cl}}^{L}} \geq\left|\xi_{L}\right|, \quad \frac{\left|\xi_{R}\right|}{\alpha_{0}} \geq\left.\xi_{R}\left(\mathbf{Y}_{1} \cdot \mathbf{e}_{x}\right)\right|_{\mathbf{x}_{\mathrm{cl}}^{R}} \geq\left|\xi_{R}\right|, \quad\left\|\mathbf{Y}_{1}\right\|_{H^{1}\left(\Gamma_{\mathrm{g}}\right)} \leq C_{1},
\end{aligned}
$$

where $\alpha_{0}$ is taken from Lemma 3.2, and $C_{1}$ depends on $\widetilde{\boldsymbol{\nu}}$ and $\alpha_{0}^{-1}$.

Proof. By (3.17), there is a $y_{0}$ in $H_{0}^{1}\left(\Gamma_{\mathrm{g}}\right)$ such that

$$
\left\langle\mu, y_{0}\right\rangle_{*}=\|\mu\|_{H^{-1}\left(\Gamma_{\mathrm{g}}\right)}, \quad\left\|y_{0}\right\|_{H^{1}\left(\Gamma_{\mathrm{g}}\right)}=1 .
$$

Now define $\mathbf{Y}_{0}=\left.y_{0} \widetilde{\boldsymbol{\nu}}\right|_{\Gamma_{\mathrm{g}}}$, where $\widetilde{\boldsymbol{\nu}}$ is taken from Lemma 3.2. Since $\left\langle\mu, y_{0}\right\rangle_{*}=\left\langle\mu, y_{0}\right\rangle_{\mathbb{M}}$ (because $\mu$ is in $\mathbb{M} \subset$ $\left.H^{-1}\left(\Gamma_{\mathrm{g}}\right)\right)$, and using $(3.1)$, we obtain $(3.30)$. 
Next, take $z$ in $H^{1}\left(\Gamma_{\mathrm{g}}\right)$ such that $z=-\operatorname{sgn}\left(\xi_{L}\right)$ at $\mathbf{x}_{\mathrm{cl}}^{L}, z=\operatorname{sgn}\left(\xi_{R}\right)$ at $\mathbf{x}_{\mathrm{cl}}^{R}$, and $z$ is linear on $\Gamma_{\mathrm{g}}$. Define

$$
w \text { in } H_{0}^{1}\left(\Gamma_{\mathrm{g}}\right) \text { such that } w=\left\{\begin{array}{r}
-y_{0}\langle\mu, z\rangle_{\mathbb{M}} /\|\mu\|_{H^{-1}\left(\Gamma_{\mathrm{g}}\right)}, \mu \neq 0 \\
0, \mu=0
\end{array}\right.
$$

Then, if $\mu \neq 0$, we have

$$
\langle\mu, w\rangle_{\mathbb{M}}=\langle\mu, w\rangle_{*}=-\langle\mu, z\rangle_{\mathbb{M}} \frac{\left\langle\mu, y_{0}\right\rangle_{*}}{\|\mu\|_{H^{-1}\left(\Gamma_{\mathrm{g}}\right)}}=-\langle\mu, z\rangle_{\mathbb{M}}, \quad \Rightarrow \quad\langle\mu, w+z\rangle_{\mathbb{M}}=0 .
$$

Now define $\mathbf{Y}_{1}=\left.(w+z) \widetilde{\boldsymbol{\nu}}\right|_{\Gamma_{\mathrm{g}}}$. It is straightforward to verify (3.31).

And we have the celebrated "inf-sup" condition

Theorem 3.12. For all $q$ in $\mathbb{Q}, \mu$ in $\mathbb{M}, \xi_{L}$ in $\mathbb{R}, \xi_{R}$ in $\mathbb{R}$, we have

$$
\sup _{\mathbf{v} \in \mathbb{V}, \mathbf{Y} \in \mathbb{Y}} \frac{b\left((\mathbf{v}, \mathbf{Y}),\left(q, \mu, \xi_{L}, \xi_{R}\right)\right)}{\|(\mathbf{v}, \mathbf{Y})\|_{\mathbb{Z}}} \geq B_{0}\left\|\left(q, \mu, \xi_{L}, \xi_{R}\right)\right\|_{\mathbb{T}},
$$

for some positive constant $B_{0}$ that depends on $\Omega, \alpha_{0}^{-1}$, and $\delta t^{-1}$.

Proof.

Step 1: Fix $q$ in $\mathbb{Q}, \mu$ in $\mathbb{M}$, and $\xi_{L}, \xi_{R}$ in $\mathbb{R}$. Let $q_{0}=\frac{1}{|\Omega|} \int_{\Omega} q$ and $\tilde{q}=q-q_{0}$. Noting that

$$
-\int_{\Omega} q \nabla \cdot \mathbf{v}+\langle\mu, \mathbf{v} \cdot \boldsymbol{\nu}\rangle_{\mathbb{M}}=-\int_{\Omega} \tilde{q} \nabla \cdot \mathbf{v}+\left\langle\mu-q_{0}, \mathbf{v} \cdot \boldsymbol{\nu}\right\rangle_{\mathbb{M}}, \quad \text { for all } \mathbf{v} \text { in } \mathbb{V},
$$

and recalling (3.13), we have

$$
\begin{aligned}
b\left((\mathbf{v}, \mathbf{Y}),\left(q, \mu, \xi_{L}, \xi_{R}\right)\right)= & -\int_{\Omega} \tilde{q} \nabla \cdot \mathbf{v}+\left\langle\mu-q_{0}, \mathbf{v} \cdot \boldsymbol{\nu}\right\rangle_{\mathbb{M}}-\frac{1}{\delta t}\langle\mu, \mathbf{Y} \cdot \boldsymbol{\nu}\rangle_{\mathbb{M}} \\
& +\left.\frac{1}{\delta t} \xi_{L}\left(\mathbf{Y} \cdot \mathbf{e}_{x}\right)\right|_{\mathbf{x}_{\mathrm{cl}}^{L}}+\left.\frac{1}{\delta t} \xi_{R}\left(\mathbf{Y} \cdot \mathbf{e}_{x}\right)\right|_{\mathbf{x}_{\mathrm{cl}}^{R}} .
\end{aligned}
$$

By Proposition 3.4 , we have $\mathbf{v}_{0}$ in $\mathbb{V}$ such that

$$
\left\langle\mu-q_{0}, \mathbf{v}_{0} \cdot \boldsymbol{\nu}\right\rangle_{\mathbb{M}}=\left\|\mu-q_{0}\right\|_{\mathbb{M}}, \quad\left\|\mathbf{v}_{0}\right\|_{H^{1}(\Omega)}=1 .
$$

Step 2: Let $\mathbf{v}$ in $\mathbb{V}$ satisfy the following divergence equation $[35,66]$

$$
\begin{aligned}
\nabla \cdot \mathbf{v} & =-\frac{\tilde{q}}{\|\tilde{q}\|_{L^{2}(\Omega)}}+\zeta, \quad \text { in } \Omega, \quad \text { where } \zeta=\frac{1}{|\Omega|} \int_{\Gamma} \mathbf{v}_{0} \cdot \nu, \\
\mathbf{v} & =\mathbf{v}_{0}, \quad \text { on } \partial \Omega .
\end{aligned}
$$

Moreover, note the following inequality:

$$
|\zeta| \leq \frac{|\Gamma|^{1 / 2}}{|\Omega|}\left\|\mathbf{v}_{0}\right\|_{0, \Gamma} \leq c_{0} \frac{|\Gamma|^{1 / 2}}{|\Omega|}
$$

Therefore, we have that $\mathbf{v}$ satisfies the bound

$$
\|\mathbf{v}\|_{H^{1}(\Omega)} \leq\left\|\left(\tilde{q} /\|\tilde{q}\|_{L^{2}(\Omega)}\right)+\zeta\right\|_{L^{2}(\Omega)}+\left\|\mathbf{v}_{0}\right\|_{H^{1 / 2}(\partial \Omega)} \leq c_{1} .
$$


Step 3: Next, apply Lemma 3.11 to construct $\mathbf{Y}=-\mathbf{Y}_{0}+\mathbf{Y}_{1}$ in $\mathbb{Y}$ (which depends on $\mu, \xi_{L}, \xi_{R}$ ). Insert $\mathbf{v}$ and $\mathbf{Y}$ into (3.35):

$$
\begin{aligned}
b\left((\mathbf{v}, \mathbf{Y}),\left(q, \mu, \xi_{L}, \xi_{R}\right)\right) & =\frac{\|\tilde{q}\|_{L^{2}(\Omega)}^{2}}{\|\tilde{q}\|_{L^{2}(\Omega)}}-\zeta \int_{\Omega} \tilde{q}+\left\|\mu-q_{0}\right\|_{\mathbb{M}}+\frac{1}{\delta t}\|\mu\|_{H^{-1}\left(\Gamma_{\mathrm{g}}\right)}+\frac{1}{\delta t}\left|\xi_{L}\right|+\frac{1}{\delta t}\left|\xi_{R}\right| \\
& \geq \frac{c_{2}}{\delta t}\left\|\left(q, \mu, \xi_{L}, \xi_{R}\right)\right\|_{\mathbb{T}},
\end{aligned}
$$

where we used (3.18). By (3.37) and Lemma 3.11, we have the bound

$$
\|(\mathbf{v}, \mathbf{Y})\|_{\mathbb{Z}} \leq c_{3}
$$

where $c_{3}$ depends on $\Omega$ and $\alpha_{0}^{-1}$. Finally, we form the ratio in (3.34), use (3.39), take the supremum, and noting that $q, \mu, \xi_{L}$, and $\xi_{R}$ are arbitrary, we get the assertion.

\subsubsection{Well-posedness}

Theorem 3.13. There exists a unique solution of the mixed formulation (3.20).

Proof. This follows from the theory in $[13,14]$ ([63], Thm. 2.3), the continuity of the forms, and Theorems 3.10 and 3.12.

\subsection{Semi-discrete energy law}

Similar to Proposition 2.8, there is an energy law for the semi-discrete formulation. Recall $\Omega^{n} \equiv \Omega\left(t_{n}\right)$, etc.

Proposition 3.14. Assume $\mathbf{f}^{n+1}$ is in $L_{\mathrm{loc}}^{2}\left(\mathbb{R}^{2}\right)$ (for $n \geq 0$ ) and the initial liquid-gas interface $\Gamma_{\mathrm{g}}(0)$ is $W^{2, \infty}$, with parametrization $\mathbf{X}^{0}$, and $\Gamma_{\mathrm{s}}(0)$ is flat with positive measure. For all $0 \leq n \leq N-1$, suppose $\delta t=t_{n+1}-t_{n}$ is uniform and the solution of (3.20) satisfies $\mathbf{u}^{n+1}$ in $H^{1}\left(\Omega^{n}\right)$, with $\mathbf{u}^{n+1} \cdot \mathbf{e}_{y}=0$ on $\Gamma_{\mathbf{s}}^{n}, \mathbf{X}^{n+1}$ in $W^{2, \infty}\left(\Gamma_{\mathrm{g}}^{n}\right)$ with $\mathbf{X}^{n+1} \cdot \mathbf{e}_{y}=0$ on $\partial \Gamma_{\mathrm{g}}^{n}, p^{n+1}$ in $L^{2}\left(\Omega^{n}\right), \Lambda_{g}^{n+1}$ in $L^{2}\left(\Gamma_{\mathrm{g}}^{n}\right), \lambda_{L}^{n+1}, \lambda_{R}^{n+1}$ in $\mathbb{R}$, where $\Gamma_{\mathrm{g}}^{n}$ is in $W^{2, \infty}$ and is parameterized by $\mathbf{X}^{n}$ and $\Gamma_{\mathrm{s}}^{n}$ is flat with positive measure. Then,

$\delta t \sum_{i=0}^{N-1}\left[\left\|\mathbf{u}^{i+1}\right\|_{H^{1}\left(\Omega^{i}\right)}^{2}+\left.\left(\mathbf{V}^{i+1} \cdot \mathbf{e}_{x}\right)^{2}\right|_{\mathbf{x}_{\mathrm{cl}}}\right]+\left|\Gamma_{\mathrm{g}}^{N}\right| \leq C\left\{\left|\Gamma_{\mathrm{g}}^{0}\right|+\delta t \sum_{i=0}^{N-1}\left[\left\|\mathbf{f}^{i+1}\right\|_{L^{2}\left(\Omega^{i}\right)}^{2}+\left.\left(\gamma_{\mathrm{s}}^{i+1}-\gamma_{\mathrm{s}, \mathrm{g}}^{i+1}\right)^{2}\right|_{\mathbf{x}_{\mathrm{cl}}}\right]\right\}$,

where $\mathbf{V}^{i+1}=\left(\mathbf{X}^{i+1}-\mathrm{id}_{\Gamma_{\mathrm{g}}^{i}}\right) / \delta$ t and the constant $C$ only depends on the domain. Recall the notation $\left.Z\right|_{\mathbf{x}_{\mathrm{cl}}}:=$ $\left.Z_{L}\right|_{\mathbf{x}_{\mathrm{cl}}^{L}}+\left.Z_{R}\right|_{\mathbf{x}_{\mathrm{cl}}^{R}}\left(\right.$ with $Z$ being any quantity at $\left.\mathbf{x}_{\mathrm{cl}}\right)$.

Proof. Proceeding as in Proposition 2.8, for a fixed time index $n$ choose $\mathbf{v}=\mathbf{u}^{n+1}$ and $q=p^{n+1}$ in (3.20) to get

$$
\frac{1}{2}\left\|D\left(\mathbf{u}^{n+1}\right)\right\|_{L^{2}\left(\Omega^{n}\right)}^{2}+\left\|\mathbf{u}^{n+1} \cdot \boldsymbol{\tau}^{n}\right\|_{L^{2}\left(\Gamma_{\mathrm{s}}^{n}\right)}^{2}+\int_{\Gamma_{\mathrm{g}}^{n}} \Lambda_{g}^{n+1}\left(\mathbf{u}^{n+1} \cdot \boldsymbol{\nu}^{n}\right)=\int_{\Omega^{n}} \mathbf{f}^{n+1} \cdot \mathbf{u}^{n+1}
$$

Next, define the discrete interface velocity $\mathbf{V}^{n+1}=\frac{\mathbf{X}^{n+1}-\mathrm{id}_{\Gamma_{\mathbf{g}}^{n}}}{\delta t}$ and choose $\mathbf{Y}=\delta t \mathbf{V}^{n+1}$ to obtain

$$
\begin{aligned}
& \int_{\Gamma_{\mathrm{g}}^{n}} \partial_{s} \mathbf{X}^{n+1} \cdot \partial_{s} \mathbf{V}^{n+1}-\int_{\Gamma_{\mathrm{g}}^{n}} \Lambda_{g}^{n+1}\left(\mathbf{V}^{n+1} \cdot \boldsymbol{\nu}^{n}\right)+\left.\left(\mathbf{V}^{n+1} \cdot \mathbf{e}_{x}\right)^{2}\right|_{\mathbf{x}_{\mathrm{cl}}}+\left.\lambda\left(\mathbf{V}^{n+1} \cdot \mathbf{e}_{x}\right)\right|_{\mathbf{x}_{\mathrm{cl}}}= \\
&\left.\left(\gamma_{\mathrm{s}}^{n+1}-\gamma_{\mathrm{s}, \mathrm{g}}^{n+1}\right)\left(\mathbf{V}^{n+1} \cdot \mathbf{e}_{x}\right)\right|_{\mathbf{x}_{\mathrm{cl}}^{L}}-\left.\left(\gamma_{\mathrm{s}}^{n+1}-\gamma_{\mathrm{s}, \mathrm{g}}^{n+1}\right)\left(\mathbf{V}^{n+1} \cdot \mathbf{e}_{x}\right)\right|_{\mathbf{x}_{\mathrm{cl}}^{R}}
\end{aligned}
$$


Setting $\xi=0$ in (3.20) implies $\left.\lambda\left(\mathbf{V}^{n+1} \cdot \mathbf{e}_{x}\right)\right|_{\mathbf{x}_{\mathrm{cl}}} \geq 0$. Thus, adding (3.41) and (3.42) and choosing $\mu=\Lambda_{g}^{n+1}$ yields

$$
\begin{aligned}
\frac{1}{2}\left\|D\left(\mathbf{u}^{n+1}\right)\right\|_{L^{2}\left(\Omega^{n}\right)}^{2}+\left\|\mathbf{u}^{n+1} \cdot \boldsymbol{\tau}^{n}\right\|_{L^{2}\left(\Gamma_{\mathrm{s}}^{n}\right)}^{2}+\int_{\Gamma_{\mathrm{g}}^{n}} \partial_{s} \mathbf{X}^{n+1} \cdot \partial_{s} \mathbf{V}^{n+1}+\left.\left(\mathbf{V}^{n+1} \cdot \mathbf{e}_{x}\right)^{2}\right|_{\mathbf{x}_{\mathrm{cl}}} \leq \\
\int_{\Omega^{n}} \mathbf{f}^{n+1} \cdot \mathbf{u}^{n+1}+\left.\left(\gamma_{\mathrm{s}}^{n+1}-\gamma_{\mathrm{s}, \mathrm{g}}^{n+1}\right)\left(\mathbf{V}^{n+1} \cdot \mathbf{e}_{x}\right)\right|_{\mathbf{x}_{\mathrm{cl}}^{L}}-\left.\left(\gamma_{\mathrm{s}}^{n+1}-\gamma_{\mathrm{s}, \mathrm{g}}^{n+1}\right)\left(\mathbf{V}^{n+1} \cdot \mathbf{e}_{x}\right)\right|_{\mathbf{x}_{\mathrm{cl}}^{R}}
\end{aligned}
$$

Next, we use a result from [5] which states that

$$
\delta t \int_{\Gamma_{\mathrm{g}}^{n}} \partial_{s} \mathbf{X}^{n+1} \cdot \partial_{s} \mathbf{V}^{n+1}=\int_{\Gamma_{\mathrm{g}}^{n}} \partial_{s} \mathbf{X}^{n+1} \cdot \partial_{s}\left(\mathbf{X}^{n+1}-\operatorname{id}_{\Gamma_{\mathrm{g}}^{n}}\right) \geq\left|\mathbf{X}^{n+1}\left(\Gamma_{\mathrm{g}}^{n}\right)\right|-\left|\Gamma_{\mathrm{g}}^{n}\right|=\left|\Gamma_{\mathrm{g}}^{n+1}\right|-\left|\Gamma_{\mathrm{g}}^{n}\right|,
$$

where $\Gamma_{\mathrm{g}}^{n+1}:=\mathbf{X}^{n+1}\left(\Gamma_{\mathrm{g}}^{n}\right)$. Using Young's inequality "with $\epsilon$ " and (3.25), we get

$$
c_{1}\left\|\mathbf{u}^{n+1}\right\|_{H^{1}\left(\Omega^{n}\right)}^{2}+\frac{\left|\Gamma_{\mathrm{g}}^{n+1}\right|-\left|\Gamma_{\mathrm{g}}^{n}\right|}{\delta t}+\left.c_{2}\left(\mathbf{V}^{n+1} \cdot \mathbf{e}_{x}\right)^{2}\right|_{\mathbf{x}_{\mathrm{cl}}} \leq c_{3}\left\|\mathbf{f}^{n+1}\right\|_{L^{2}\left(\Omega^{n}\right)}^{2}+\left.c_{4}\left(\gamma_{\mathrm{s}}^{n+1}-\gamma_{\mathrm{s}, \mathrm{g}}^{n+1}\right)^{2}\right|_{\mathbf{x}_{\mathrm{cl}}},
$$

where the constants only depend on the domain $\Omega^{n}$. Indeed, the constants do not depend on $\delta t$. Multiplying through by $\delta t$ and summing gives the assertion.

\section{Fully Discrete FORMulation}

We extend the results of Section 3 to the fully discrete setting (i.e. space is now discretized), the main results being Theorem 4.5, Lemmas 4.9 and 4.10, and the discrete energy law (Prop. 4.11).

\subsection{Triangulation}

The fully discrete scheme consists of applying a spatial discretization to the semi-discrete formulation (see Sect. 3.3). As usual, we approximate the domain $\Omega$ by a triangulated domain $\Omega_{h}$. The triangulation is denoted by $\mathcal{T}_{h}$, where $h$ is the longest edge of all the triangles. We assume $\mathcal{T}_{h}$ is conforming, shape regular [12], and satisfies

$$
\Omega_{h}=\cup_{T \in \mathcal{T}_{h}} T .
$$

The boundary of the triangulation is denoted $\partial \Omega_{h}=\overline{\Gamma_{\mathrm{g}, \mathrm{h}}} \cup \overline{\Gamma_{\mathrm{s}, \mathrm{h}}}$ and the edges of all triangles that lie on $\Gamma_{\mathrm{g}, \mathrm{h}}$ are assumed to be quadratic curves (i.e. second order geometric approximation). Furthermore, we denote the set of curved edges of $\Gamma_{\mathrm{g}, \mathrm{h}}$ as $\mathcal{E}_{h}$, where

$$
\Gamma_{\mathrm{g}, \mathrm{h}}=\cup_{E \in \mathcal{E}_{h}} E,
$$

and $\overline{\Gamma_{\mathrm{g}, \mathrm{h}}} \cap \overline{\Gamma_{\mathrm{s}, \mathrm{h}}}=\left\{\mathbf{x}_{\mathrm{cl}}^{L}, \mathbf{x}_{\mathrm{cl}}^{R}\right\}$ consists of two vertices of the triangulation $\mathcal{T}_{h}$, which represent the left and right contact points.

\subsection{Finite element spaces}

We introduce the finite element spaces used to approximate $\mathbb{V}, \mathbb{Y}, \mathbb{Q}$, and $\mathbb{M}$. Let $\mathcal{P}_{k}$ be the space of polynomials of degree $\leq k$ on the standard reference triangle $\widehat{T}$ or standard reference edge $\widehat{E}$. Let $\Psi_{T}: \widehat{T} \rightarrow T$ be the iso-parametric $\mathcal{P}_{2}$ mapping from the reference triangle to a triangle in $\mathcal{T}_{h}$, and let $\Psi_{E}: \widehat{E} \rightarrow E$ be similarly defined for edges $E$ in $\mathcal{E}_{h}$. Then the finite element spaces are defined as

$$
V_{k}:=\left\{\mathbf{v} \in C\left(\overline{\Omega_{h}}\right): \mathbf{v} \circ \Psi_{T} \in \mathcal{P}_{k}(\widehat{T}), \text { for all } T \in \mathcal{T}_{h}\right\},
$$


i.e. the space of continuous vector basis functions, whose components are piecewise polynomials of degree $\leq k$ on the reference triangle $\widehat{T}$. Similarly,

$$
Y_{k}:=\left\{\mathbf{Y} \in C\left(\overline{\Gamma_{\mathrm{g}, \mathrm{h}}}\right): \mathbf{Y} \circ \Psi_{E} \in \mathcal{P}_{k}(\widehat{E}), \text { for all } E \in \mathcal{E}_{h}\right\}
$$

Next, we have the scalar valued spaces

$$
\begin{aligned}
Q_{k} & :=\left\{q \in C\left(\overline{\Omega_{h}}\right): q \circ \Psi_{T} \in \mathcal{P}_{k}(\widehat{T}), \text { for all } T \in \mathcal{T}_{h}\right\}, \\
M_{0} & :=\left\{\mu \in L^{2}\left(\Gamma_{\mathrm{g}, \mathrm{h}}\right): \mu \circ \Psi_{E} \in \mathcal{P}_{0}(\widehat{E}), \text { for all } E \in \mathcal{E}_{h}\right\}, \\
M_{k} & :=\left\{\mu \in C\left(\overline{\Gamma_{\mathrm{g}, \mathrm{h}}}\right): \mu \circ \Psi_{E} \in \mathcal{P}_{k}(\widehat{E}), \text { for all } E \in \mathcal{E}_{h}\right\}, \quad k \geq 1 .
\end{aligned}
$$

Note that $V_{k} \subset H^{1}\left(\Omega_{h}\right), Y_{k} \subset H^{1}\left(\Gamma_{\mathrm{g}, \mathrm{h}}\right), Q_{k} \subset L^{2}\left(\Omega_{h}\right), M_{0}, M_{k} \subset\left(H^{1 / 2}\left(\Gamma_{\mathrm{g}, \mathrm{h}}\right)\right)^{*}$, for all $k \geq 1$.

Let $\mathbb{V}_{h}, \mathbb{Y}_{h}, \mathbb{Q}_{h}$, and $\mathbb{M}_{h}$ be conforming approximations of $\mathbb{V}, \mathbb{Y}, \mathbb{Q}$, and $\mathbb{M}$, defined by:

$$
\begin{aligned}
\mathbb{V}_{h} & :=\left\{\mathbf{v} \in V_{2}: \mathbf{v} \cdot \mathbf{e}_{y}=0, \text { on } \Gamma_{\mathrm{s}}\right\}, \\
\mathbb{Y}_{h} & :=\left\{\mathbf{v} \in Y_{2}: \mathbf{Y} \cdot \mathbf{e}_{y}=0, \text { at } \partial \Gamma_{\mathrm{g}, \mathrm{h}}\right\}, \\
\mathbb{Q}_{h} & :=Q_{1}, \\
\mathbb{M}_{h} & :=M_{0} \text { or } M_{1} .
\end{aligned}
$$

These finite element spaces are equipped with the standard Sobolev norms. Of course, the convex set is simply $\mathcal{K}_{h} \equiv \mathcal{K}=[-1,1] \subset \mathbb{R}$.

Remark 4.1. The space $\mathbb{Y}_{h}$ matches the $\mathcal{P}_{2}$ iso-parametric mapping. Thus, updating the discrete domain over consecutive time-steps is straightforward (see Rem. 3.5).

\subsection{Mixed formulation}

The analogous discrete bilinear and linear forms to (3.12)-(3.14) are as follows:

$$
\begin{aligned}
a_{h}\left(\left(\mathbf{u}_{h}, \mathbf{W}_{h}\right),\left(\mathbf{v}_{h}, \mathbf{Y}_{h}\right)\right)= & \int_{\Omega_{h}} D\left(\mathbf{u}_{h}\right): \nabla \mathbf{v}_{h}+\int_{\Gamma_{\mathrm{s}}}\left(\mathbf{u}_{h} \cdot \boldsymbol{\tau}\right)\left(\mathbf{v}_{h} \cdot \boldsymbol{\tau}\right)+\frac{1}{\delta t} \int_{\Gamma_{\mathrm{g}, \mathrm{h}}} \partial_{s} \mathbf{W}_{h} \cdot \partial_{s} \mathbf{Y}_{h} \\
& +\left.\frac{1}{\delta t^{2}}\left(\mathbf{W}_{h} \cdot \mathbf{e}_{x}\right)\left(\mathbf{Y}_{h} \cdot \mathbf{e}_{x}\right)\right|_{\mathbf{x}_{\mathrm{cl}}^{L}}+\left.\frac{1}{\delta t^{2}}\left(\mathbf{W}_{h} \cdot \mathbf{e}_{x}\right)\left(\mathbf{Y}_{h} \cdot \mathbf{e}_{x}\right)\right|_{\mathbf{x}_{\mathrm{cl}}^{R}}, \\
b_{h}\left(\left(\mathbf{v}_{h}, \mathbf{Y}_{h}\right),\left(q_{h}, \mu_{h}, \xi_{L}, \xi_{R}\right)\right)= & -\int_{\Omega_{h}} q_{h} \nabla \cdot \mathbf{v}_{h}+\int_{\Gamma_{\mathrm{g}, \mathrm{h}}} \mu_{h}\left(\mathbf{v}_{h} \cdot \boldsymbol{\nu}_{h}\right)-\frac{1}{\delta t} \int_{\Gamma_{\mathrm{g}, \mathrm{h}}} \mu_{h}\left(\mathbf{Y}_{h} \cdot \boldsymbol{\nu}_{h}\right) \\
& +\left.\frac{1}{\delta t} \xi_{L}\left(\mathbf{Y}_{h} \cdot \mathbf{e}_{x}\right)\right|_{\mathbf{x}_{\mathrm{cl}}^{L}}+\left.\frac{1}{\delta t} \xi_{R}\left(\mathbf{Y}_{h} \cdot \mathbf{e}_{x}\right)\right|_{\mathbf{x}_{\mathrm{cl}}^{R}}, \\
F_{h}\left(\left(\mathbf{v}_{h}, \mathbf{Y}_{h}\right)\right)= & \int_{\Omega_{h}} \mathbf{f} \cdot \mathbf{v}_{h}+\left.\frac{1}{\delta t}\left(\gamma_{\mathrm{s}}-\gamma_{\mathrm{s}, \mathrm{g}}\right)\left(\mathbf{Y}_{h} \cdot \mathbf{e}_{x}\right)\right|_{\mathbf{x}_{\mathrm{cl}}^{L}}-\left.\frac{1}{\delta t}\left(\gamma_{\mathrm{s}}-\gamma_{\mathrm{s}, \mathrm{g}}\right)\left(\mathbf{Y}_{h} \cdot \mathbf{e}_{x}\right)\right|_{\mathbf{x}_{\mathrm{cl}}^{R}} \\
& +\left.\frac{1}{\delta t^{2}}\left(\mathrm{id}_{\Gamma_{\mathrm{g}, \mathrm{h}}} \cdot \mathbf{e}_{x}\right)\left(\mathbf{Y}_{h} \cdot \mathbf{e}_{x}\right)\right|_{\mathbf{x}_{\mathrm{cl}}^{L}}+\left.\frac{1}{\delta t^{2}}\left(\mathrm{id}_{\Gamma_{\mathrm{g}, \mathrm{h}}} \cdot \mathbf{e}_{x}\right)\left(\mathbf{Y}_{h} \cdot \mathbf{e}_{x}\right)\right|_{\mathbf{x}_{\mathrm{cl}}^{R}}, \\
G_{h}\left(\left(q_{h}, \mu_{h}, \xi_{L}, \xi_{R}\right)\right)= & -\frac{1}{\delta t} \int_{\Gamma_{\mathrm{g}, \mathrm{h}}} \mu_{h}\left(\mathrm{id}_{\Gamma_{\mathrm{g}, \mathrm{h}}} \cdot \boldsymbol{\nu}_{h}\right)+\left.\frac{1}{\delta t} \xi_{L}\left(\mathrm{id}_{\Gamma_{\mathrm{g}, \mathrm{h}}} \cdot \mathbf{e}_{x}\right)\right|_{\mathbf{x}_{\mathrm{cl}}^{L}}+\left.\frac{1}{\delta t} \xi_{R}\left(\mathrm{id}_{\Gamma_{\mathrm{g}, \mathrm{h}}} \cdot \mathbf{e}_{x}\right)\right|_{\mathbf{x}_{\mathrm{cl}}^{R}},
\end{aligned}
$$

for all $\mathbf{v}_{h}$ in $\mathbb{V}_{h}, \mathbf{Y}_{h}$ in $\mathbb{Y}_{h}, q_{h}$ in $\mathbb{Q}_{h}, \mu_{h}$ in $\mathbb{M}_{h}$, and $\xi_{L}, \xi_{R}$ in $\mathbb{R}$. Note that $\Gamma_{\mathrm{s}, \mathrm{h}} \equiv \Gamma_{\mathrm{s}}$ because it is flat, so then the discrete tangent vector satisfies $\boldsymbol{\tau}_{h} \equiv \boldsymbol{\tau}=\mathbf{e}_{x}$ on $\Gamma_{\mathrm{s}}$.

The discrete version of the product space (3.15) is

$$
\mathbb{Z}_{h}=\mathbb{V}_{h} \times \mathbb{Y}_{h}, \quad \mathbb{T}_{h}=\mathbb{Q}_{h} \times \mathbb{M}_{h} \times \mathcal{K} \times \mathcal{K},
$$


and the discrete version of the norms (3.16)-(3.18) are defined in the obvious way. Thus, at each time-step, the mixed formulation is: find $\left(\mathbf{u}_{h}, \mathbf{W}_{h}\right)$ in $\mathbb{Z}_{h}$ and $\left(p_{h}, \Lambda_{g, h}, \lambda_{L, h}, \lambda_{R, h}\right)$ in $\mathbb{T}_{h}$ such that

$$
\begin{aligned}
& a_{h}\left(\left(\mathbf{u}_{h}, \mathbf{W}_{h}\right),\left(\mathbf{v}_{h}, \mathbf{Y}_{h}\right)\right)+b_{h}\left(\left(\mathbf{v}_{h}, \mathbf{Y}_{h}\right),\left(p_{h}, \Lambda_{g, h}, \lambda_{L, h}, \lambda_{R, h}\right)\right)=F_{h}\left(\left(\mathbf{v}_{h}, \mathbf{Y}_{h}\right)\right), \\
& \quad b_{h}\left(\left(\mathbf{u}_{h}, \mathbf{W}_{h}\right),\left(q_{h}, \mu_{h}, \xi_{L}-\lambda_{L, h}, \xi_{R}-\lambda_{R, h}\right)\right) \leq G_{h}\left(\left(q_{h}, \mu_{h}, \xi_{L}-\lambda_{L, h}, \xi_{R}-\lambda_{R, h}\right)\right),
\end{aligned}
$$

for all $\left(\mathbf{v}_{h}, \mathbf{Y}_{h}\right)$ in $\mathbb{Z}_{h}$ and $\left(q_{h}, \mu_{h}, \xi_{L}, \xi_{R}\right)$ in $\mathbb{T}_{h}$. The solution of (4.10) is iterated for the full time-varying simulation (see Rem. 3.5). In particular, we update the domain boundary with $\mathbf{X}_{h}^{n+1}:=\mathbf{W}_{h}^{n+1}$, then use a smooth domain deformation to update the interior vertices of the mesh (see [32] for a similar approach).

\subsection{Variational crime}

Using a polygonal, or even piecewise quadratic, approximation of the domain boundary $\partial \Omega_{h}$ introduces an additional geometric error. This has been considered in $[7,12,43,69]$ and is now a classical issue. Thus, when comparing the solutions of the semi-discrete and fully discrete problems, there are two terms to consider. The first is the energy error due to the usual finite dimensional space approximation. The other term is related to the domain approximation (i.e. a variational crime).

At the initial time step, it is reasonable to assume that the quadratic nodes (vertices) of the approximating curve $\Gamma_{\mathrm{g}, \mathrm{h}}^{0}$ interpolate the true (initial) curve $\Gamma_{\mathrm{g}}^{0}$. Of course, the semi-discrete and fully discrete evolution schemes will give different results for the interfaces $\Gamma_{\mathrm{g}}^{n}$ and $\Gamma_{\mathrm{g}, \mathrm{h}}^{n}$ (for $n>0$ ), respectively, because of the accumulated spatial discretization error. Understanding this requires a full time-dependent analysis, which we do not give here. Hence, we assume $\Gamma_{\mathrm{g}}$ is approximated by $\Gamma_{\mathrm{g}, \mathrm{h}}$, at all time-steps, in the following sense:

$$
\begin{gathered}
\sup _{x \in \Gamma_{\mathrm{g}}} \inf _{y \in \Gamma_{\mathrm{g}, \mathrm{h}}}|x-y| \leq c h^{k+1}, \\
\left\|\boldsymbol{\nu} \circ \boldsymbol{\Phi}-\boldsymbol{\nu}_{h}\right\|_{L^{\infty}\left(\Gamma_{\mathrm{g}, \mathrm{h}}\right)} \leq c h^{k},
\end{gathered}
$$

where $\Gamma_{\mathrm{g}}$ is assumed to be $W^{k+1, \infty}$ (for $k=1$ or 2), $\boldsymbol{\nu}$ is the unit normal on $\Gamma_{\mathrm{g}}, \boldsymbol{\nu}_{h}$ is the unit normal on $\Gamma_{\mathrm{g}, \mathrm{h}}$,

and $\boldsymbol{\Phi}: \Gamma_{\mathrm{g}, \mathrm{h}} \rightarrow \Gamma_{\mathrm{g}}$ is a suitable map from $\Gamma_{\mathrm{g}, \mathrm{h}}$ to $\Gamma_{\mathrm{g}}$ (see $[6,43,69]$ for how this can be constructed). Note that since $\Gamma_{\mathrm{s}}$ is flat, we have $\Gamma_{\mathrm{s}} \equiv \Gamma_{\mathrm{s}, \mathrm{h}}$. We emphasize that iso-parametric elements are needed to get improved $L^{2}$ error estimates for velocity and position because the normal vector appears in the weak formulation.

Therefore, since the variational crime argument is classical, we avoid discussing the technicalities associated with approximating the domain. In particular, we shall assume the semi-discrete and fully discrete problems are defined over the same domain. Hence, we take $\Omega_{h} \equiv \Omega, \Gamma_{\mathrm{g}, \mathrm{h}} \equiv \Gamma_{\mathrm{g}}, \Gamma_{\mathrm{s}, \mathrm{h}} \equiv \Gamma_{\mathrm{s}}$, and

$$
\mathbb{V}_{h} \subset \mathbb{V}, \quad \mathbb{Y}_{h} \subset \mathbb{Y}, \quad \mathbb{Q}_{h} \subset \mathbb{Q}, \quad \mathbb{M}_{h} \subset \mathbb{M} \text {. }
$$

Remark 4.2. We make one exception to the above simplification and maintain an important technicality. The normal vector of the discrete domain $\boldsymbol{\nu}_{h}$ is discontinuous, which impacts the duality pairing $\left\langle\mu, \mathbf{v} \cdot \boldsymbol{\nu}_{h}\right\rangle_{\mathbb{M}}$. This directly affects the proof of the discrete inf-sup condition, namely Lemmas 4.9 and 4.10. For the error estimates in Section 5, we do not make explicit note of the normal vector approximation.

Alternatively, one could change the discrete formulation so that $\boldsymbol{\nu}_{h}$ is continuous, i.e. replace the true discrete normal of $\Gamma_{\mathrm{g}, \mathrm{h}}$ with a continuous approximation [30]. We do not pursue this here.

\subsection{Stable formulation}

Because the finite element spaces are conforming (4.12), we automatically obtain the following results from Lemma 3.6 and Theorem 3.10. 
Lemma 4.3 (Continuity). Let $\Omega_{h}$ be a bounded polyhedral domain in $\mathbb{R}^{d}$ with possibly curved boundary facets. Then there are positive constants $C_{a}, C_{b}, C_{F}$, and $C_{G}$ that depend on $\Omega_{h}$ and $\delta t^{-1}$ such that

$$
\begin{aligned}
a_{h}\left(\left(\mathbf{u}_{h}, \mathbf{W}_{h}\right),\left(\mathbf{v}_{h}, \mathbf{Y}_{h}\right)\right) & \leq C_{a}\left\|\left(\mathbf{u}_{h}, \mathbf{W}_{h}\right)\right\|_{\mathbb{Z}}\left\|\left(\mathbf{v}_{h}, \mathbf{Y}_{h}\right)\right\|_{\mathbb{Z}}, \\
b_{h}\left(\left(\mathbf{u}_{h}, \mathbf{W}_{h}\right),\left(q_{h}, \mu_{h}, \xi_{L}, \xi_{R}\right)\right) & \leq C_{b}\left\|\left(\mathbf{u}_{h}, \mathbf{W}_{h}\right)\right\|_{\mathbb{Z}}\left\|\left(q_{h}, \mu_{h}, \xi_{L}, \xi_{R}\right)\right\|_{\mathbb{T}}, \\
F_{h}\left(\left(\mathbf{v}_{h}, \mathbf{Y}_{h}\right)\right) & \leq C_{F}\left\|\left(\mathbf{v}_{h}, \mathbf{Y}_{h}\right)\right\|_{\mathbb{Z}}, \\
G_{h}\left(\left(q_{h}, \mu_{h}, \xi_{L}, \xi_{R}\right)\right) & \leq C_{G}\left\|\left(q_{h}, \mu_{h}, \xi_{L}, \xi_{R}\right)\right\|_{\mathbb{T}},
\end{aligned}
$$

for all $\left(\mathbf{u}_{h}, \mathbf{W}_{h}\right),\left(\mathbf{v}_{h}, \mathbf{Y}_{h}\right)$ in $\mathbb{Z}_{h}$ and $\left(q_{h}, \mu_{h}, \xi_{L}, \xi_{R}\right)$ in $\mathbb{T}_{h}$.

Theorem 4.4. Assume the hypothesis of Lemmas 3.7 and 3.9. For all $\mathbf{v}_{h}$ in $\mathbb{V}_{h}$ and all $\mathbf{Y}_{h}$ in $\mathbb{Y}_{h}$, we have

$$
a_{h}\left(\left(\mathbf{v}_{h}, \mathbf{Y}_{h}\right),\left(\mathbf{v}_{h}, \mathbf{Y}_{h}\right)\right) \geq C\left(\left\|\mathbf{v}_{h}\right\|_{H^{1}\left(\Omega_{h}\right)}^{2}+\left\|\mathbf{Y}_{h}\right\|_{H^{1}\left(\Gamma_{\mathrm{g}, \mathrm{h}}\right)}^{2}\right)=C\left\|\left(\mathbf{v}_{h}, \mathbf{Y}_{h}\right)\right\|_{\mathbb{Z}}^{2}
$$

for some positive constant $C$ that depends on $\Omega_{h}$ and $\delta t^{-1}($ for $\delta t \leq 1)$.

To ensure the stability of the fully discrete solution, we again need the "inf-sup" condition.

Theorem 4.5. For all $q_{h}$ in $\mathbb{Q}_{h}, \mu_{h}$ in $\mathbb{M}_{h}, \xi_{L}$ in $\mathbb{R}, \xi_{R}$ in $\mathbb{R}$, and for $h$ sufficiently small, we have

$$
\sup _{\mathbf{v}_{h} \in \mathbb{V}_{h}, \mathbf{Y}_{h} \in \mathbb{Y}_{h}} \frac{b_{h}\left(\left(\mathbf{v}_{h}, \mathbf{Y}_{h}\right),\left(q_{h}, \mu_{h}, \xi_{L}, \xi_{R}\right)\right)}{\left\|\left(\mathbf{v}_{h}, \mathbf{Y}_{h}\right)\right\|_{\mathbb{Z}}} \geq \beta\left\|\left(q_{h}, \mu_{h}, \xi_{L}, \xi_{R}\right)\right\|_{\mathbb{T}},
$$

for some positive constant $\beta$ that depends on $\Omega_{h}, \alpha_{0}^{-1}$, and $\delta t^{-1}$.

Before proving Theorem 4.5, we prove some intermediate results.

Proposition 4.6 (Piecewise Constant $\mathbb{M}_{h}$ ). Let $\Omega$ be piecewise smooth, such that $\Gamma_{\mathrm{g}}$ is $W^{2, \infty}$ and $\Gamma_{\mathrm{s}}$ is flat. Let $\Omega_{h}$ be a piecewise quadratic approximation of $\Omega$. Given $\mu_{h}$ in $M_{0}$, there is a $\mathbf{v}_{h}$ in $\mathbb{V}_{h}$ such that

$$
\int_{\Gamma_{\mathrm{g}, \mathrm{h}}} \mu_{h}\left(\mathbf{v}_{h} \cdot \boldsymbol{\nu}_{h}\right) \geq C h^{1 / 2}\left\|\mu_{h}\right\|_{L^{2}\left(\Gamma_{\mathrm{g}, \mathrm{h}}\right)}, \quad\left\|\mathbf{v}_{h}\right\|_{H^{1}\left(\Omega_{h}\right)}=1
$$

for some independent constant $C>0$ depending only on $\Omega$, and for $h$ sufficiently small.

Proof. Take $\mu_{h}$ in $M_{0}$, i.e. $\mu_{h}$ is constant on each edge segment of $\Gamma_{\mathrm{g}, \mathrm{h}}$. Let $E$ be a quadratic edge segment of $\Gamma_{\mathrm{g}, \mathrm{h}}$, and denote the midpoint by $\mathbf{x}_{E}$. Let $b_{E}$ be a quadratic "bubble" function on $E$, i.e. $b_{E}$ is in $\mathbb{V}_{h}$ such that all its nodal values vanish except at $\mathbf{x}_{E}$ where $b_{E}\left(\mathbf{x}_{E}\right)=1$. Let $\mathbf{v}_{h}$ in $\mathbb{V}_{h}$, and define it on $\Gamma_{\mathrm{g}, \mathrm{h}}$ by

$$
\left.\mathbf{v}_{h}\right|_{E}=\mu_{h}\left(\left.\nu_{h}\right|_{\mathbf{x}_{E}}\right) b_{E}, \quad \text { for all } E \subset \Gamma_{\mathrm{g}, \mathrm{h}},
$$

and set $\mathbf{v}_{h}=\mathbf{0}$ on $\Gamma_{\mathrm{s}, \mathrm{h}}$. This gives the following bound

$$
\left\|\mathbf{v}_{h}\right\|_{L^{2}\left(\Gamma_{\mathrm{g}, \mathrm{h}}\right)}^{2}=\int_{\Gamma_{\mathrm{g}, \mathrm{h}}}\left|\mathbf{v}_{h}\right|^{2}=\sum_{E \subset \Gamma_{\mathrm{g}, \mathrm{h}}} \mu_{h}^{2} \int_{E} b_{E}^{2} \leq c_{1} \sum_{E \subset \Gamma_{\mathrm{g}, \mathrm{h}}} \mu_{h}^{2}|E|=c_{1}\left\|\mu_{h}\right\|_{L^{2}\left(\Gamma_{\mathrm{g}, \mathrm{h}}\right)}^{2} .
$$

Next, let $\tilde{\mathbf{v}}$ be the harmonic extension of $\mathbf{v}_{h}$ to all of $\Omega_{h}$. With a slight abuse of notation, let $\mathbf{v}_{h}=\Pi_{h} \tilde{\mathbf{v}}$, where $\Pi_{h}$ is the Scott-Zhang interpolant [59], onto $\mathbb{V}_{h}$, that preserves boundary values on $\partial \Omega_{h}$. Then, by (4.19) and a trace and inverse estimate, we have

$$
\begin{aligned}
\left\|\mathbf{v}_{h}\right\|_{H^{1}\left(\Omega_{h}\right)} & \leq c_{2}\left\|\mathbf{v}_{h}\right\|_{H^{1 / 2}\left(\partial \Omega_{h}\right)}=c_{2}\left\|\mathbf{v}_{h}\right\|_{H^{1 / 2}\left(\Gamma_{\mathrm{g}, \mathrm{h}}\right)} \leq c_{3} h^{-1 / 2}\left\|\mathbf{v}_{h}\right\|_{L^{2}\left(\Gamma_{\mathrm{g}, \mathrm{h}}\right)} \\
& \leq c_{4} h^{-1 / 2}\left\|\mu_{h}\right\|_{L^{2}\left(\Gamma_{\mathrm{g}, \mathrm{h}}\right)} .
\end{aligned}
$$


Therefore, since $\left.\boldsymbol{\nu}_{h}\left(\mathbf{x}_{E}\right) \cdot \boldsymbol{\nu}_{h}\right|_{E} \geq \frac{1}{2}$ for $h$ sufficiently small, we arrive at

$$
\frac{\int_{\Gamma_{\mathrm{g}, \mathrm{h}}} \mu_{h}\left(\mathbf{v}_{h} \cdot \boldsymbol{\nu}_{h}\right)}{\left\|\mathbf{v}_{h}\right\|_{H^{1}\left(\Omega_{h}\right)}}=\frac{\sum_{E \subset \Gamma_{\mathrm{g}, \mathrm{h}}} \mu_{h}^{2} \int_{E}\left(\boldsymbol{\nu}_{h}\left(\mathbf{x}_{E}\right) \cdot \boldsymbol{\nu}_{h}\right) b_{E}}{\left\|\mathbf{v}_{h}\right\|_{H^{1}\left(\Omega_{h}\right)}} \geq C h^{1 / 2}\left\|\mu_{h}\right\|_{L^{2}\left(\Gamma_{\mathrm{g}, \mathrm{h}}\right)},
$$

which gives the assertion.

Proposition 4.7 (Continuous Piecewise Linear $\mathbb{M}_{h}$ ). Assume the hypothesis of Proposition 4.6. Given $\mu_{h}$ in $M_{1}$, there is a $\mathbf{v}_{h}$ in $\mathbb{V}_{h}$ such that

$$
\int_{\Gamma_{\mathrm{g}, \mathrm{h}}} \mu_{h}\left(\mathbf{v}_{h} \cdot \boldsymbol{\nu}_{h}\right) \geq C h^{1 / 2}\left\|\mu_{h}\right\|_{L^{2}\left(\Gamma_{\mathrm{g}, \mathrm{h}}\right)}, \quad\left\|\mathbf{v}_{h}\right\|_{H^{1}\left(\Omega_{h}\right)}=1
$$

for some independent constant $C>0$ depending only on $\Omega$, and for $h$ sufficiently small.

Proof. Recall the smoothed, extended vector field $\widetilde{\boldsymbol{\nu}}_{E}$ from Lemma 3.2. Since $\Omega_{h}$ approximates $\Omega$ in the sense of (4.11), we can define $\boldsymbol{\nu}_{s}$ to be the standard piecewise linear interpolant of $\widetilde{\boldsymbol{\nu}}_{E}$ over $\Omega_{h}$ (using a diffeomorphism $\Phi: \Omega_{h} \rightarrow \Omega$ if necessary). Then we have, for $h$ sufficiently small,

$$
\begin{aligned}
\boldsymbol{\nu}_{s} \in V_{1}, \quad \boldsymbol{\nu}_{s} \cdot \boldsymbol{\nu}_{h} & \geq a_{1}>0, \text { on } \Gamma_{\mathrm{g}, \mathrm{h}}, \quad \boldsymbol{\nu}_{s} \cdot \boldsymbol{\nu}_{h}=0, \text { on } \Gamma_{\mathrm{s}, \mathrm{h}}, \\
\left\|\boldsymbol{\nu}_{s}\right\|_{W^{1, \infty}\left(\partial \Omega_{h}\right)} & \leq a_{2}, \quad\left\|\boldsymbol{\nu}_{s}\right\|_{W^{1, \infty}\left(\Omega_{h}\right)} \leq a_{3},
\end{aligned}
$$

for some constants $a_{1}, a_{2}, a_{3}$ independent of $h$, where $\boldsymbol{\nu}_{h}$ is the outward unit normal vector of the piecewise quadratic curve $\partial \Omega_{h}$. Take $\mu_{h}$ in $M_{1}$ and extend its definition to all of $\partial \Omega_{h}$ in a smooth way so that

$$
\left\|\mu_{h}\right\|_{H^{1 / 2}\left(\partial \Omega_{h}\right)} \leq c_{0}\left\|\mu_{h}\right\|_{H^{1 / 2}\left(\Gamma_{\mathrm{g}, \mathrm{h}}\right)} .
$$

Next, similar to Proposition 4.6, we extend $\mu_{h}$ to $\Omega_{h}$ such that $\left\|\mu_{h}\right\|_{H^{1}\left(\Omega_{h}\right)} \leq c_{1}\left\|\mu_{h}\right\|_{H^{1 / 2}\left(\Gamma_{\mathrm{g}, \mathrm{h}}\right)}$, where $\mu_{h}$ is in $Q_{1}$. Thus, by an inverse estimate, $\mu_{h}$ satisfies $\left\|\mu_{h}\right\|_{H^{1}\left(\Omega_{h}\right)} \leq c_{2} h^{-1 / 2}\left\|\mu_{h}\right\|_{L^{2}\left(\Gamma_{\mathrm{g}, \mathrm{h}}\right)}$.

Define $\mathbf{v}_{h}$ in $\mathbb{V}_{h}$ by $\mathbf{v}_{h}:=\mu_{h} \boldsymbol{\nu}_{s}$. By the properties (4.23) of $\boldsymbol{\nu}_{s}$, we have that

$$
\left\|\mathbf{v}_{h}\right\|_{H^{1}\left(\Omega_{h}\right)} \leq c_{3}\left\|\mu_{h}\right\|_{H^{1}\left(\Omega_{h}\right)} \leq c_{4} h^{-1 / 2}\left\|\mu_{h}\right\|_{L^{2}\left(\Gamma_{\mathrm{g}, \mathrm{h}}\right)} .
$$

Therefore, we obtain

$$
\frac{\int_{\Gamma_{\mathrm{g}, \mathrm{h}}} \mu_{h}\left(\mathbf{v}_{h} \cdot \boldsymbol{\nu}_{h}\right)}{\left\|\mathbf{v}_{h}\right\|_{H^{1}\left(\Omega_{h}\right)}} \geq \frac{a_{1}}{c_{4}} h^{1 / 2} \frac{\int_{\Gamma_{\mathrm{g}, \mathrm{h}}} \mu_{h}^{2}}{\left\|\mu_{h}\right\|_{L^{2}\left(\Gamma_{\mathrm{g}, \mathrm{h}}\right)}}=c_{5} h^{1 / 2}\left\|\mu_{h}\right\|_{L^{2}\left(\Gamma_{\mathrm{g}, \mathrm{h}}\right)},
$$

which gives the assertion.

Proposition 4.8 (Matching function in $\mathbb{Y}_{h}$ ). Assume the hypothesis of Proposition 4.6. Given $\mu_{h}$ in $\mathbb{M}_{h}$, where $\mathbb{M}_{h}=M_{0}$ or $\mathbb{M}_{h}=M_{1}$, there is a $\mathbf{Y}_{h}$ in $\mathbb{Y}_{h} \cap H_{0}^{1}\left(\Gamma_{\mathrm{g}, \mathrm{h}}\right)$ such that

$$
\int_{\Gamma_{\mathrm{g}, \mathrm{h}}} \mu_{h}\left(\mathbf{Y}_{h} \cdot \boldsymbol{\nu}_{h}\right) \geq C h\left\|\mu_{h}\right\|_{L^{2}\left(\Gamma_{\mathrm{g}, \mathrm{h}}\right)}, \quad\left\|\mathbf{Y}_{h}\right\|_{H^{1}\left(\Gamma_{\mathrm{g}, \mathrm{h}}\right)}=1,
$$

for some independent constant $C>0$ depending only on $\Gamma_{\mathrm{g}}$.

Proof. First note that the finite element space $\mathbb{Y}_{h}$ is just the restriction of $\mathbb{V}_{h}$ to $\Gamma_{\mathrm{g}, \mathrm{h}}$. Thus, it is a straightforward modification of the proofs in Propositions 4.6 and 4.7 to construct $\mathbf{Y}_{h}$. In particular, the zero boundary values for $\mathbf{Y}_{h}$ do not pose a problem.

We now prove an intermediate inf-sup result. 
Lemma 4.9 (Inf-Sup For Boundary Multiplier). Assume the hypothesis of Proposition 4.6. Then there is a constant $\beta_{2}>0$, depending on $\Omega$, such that, for $h$ sufficiently small,

$$
\sup _{\mathbf{v}_{h} \in \mathbb{V}_{h}} \frac{\int_{\Gamma_{\mathrm{g}, \mathrm{h}}} \mu_{h}\left(\mathbf{v}_{h} \cdot \boldsymbol{\nu}_{h}\right)}{\left\|\mathbf{v}_{h}\right\|_{H^{1}\left(\Omega_{h}\right)}} \geq \beta_{2}\left\|\mu_{h}\right\|_{\mathbb{M}}, \quad \text { for all } \mu_{h} \text { in } \mathbb{M}_{h},
$$

where $\mathbb{M}_{h}=M_{0}$ or $\mathbb{M}_{h}=M_{1}$.

Proof. Let $\mu_{h}$ in $\mathbb{M}_{h}$. If $\mathbb{M}_{h}=M_{0}$, then let $\mathbf{u}_{h}$ be given by Proposition 4.6. Else, if $\mathbb{M}_{h}=M_{1}$, then let $\mathbf{u}_{h}$ be given by Proposition 4.7. Therefore,

$$
\int_{\Gamma_{\mathrm{g}, \mathrm{h}}} \mu_{h}\left(\mathbf{u}_{h} \cdot \boldsymbol{\nu}_{h}\right) \geq C h^{1 / 2}\left\|\mu_{h}\right\|_{L^{2}\left(\Gamma_{\mathrm{g}, \mathrm{h}}\right)}, \quad\left\|\mathbf{u}_{h}\right\|_{H^{1}\left(\Omega_{h}\right)}=1
$$

Hence, we must account for the $h^{1 / 2}$ weighting.

Let $\boldsymbol{\nu}$ be the $W^{1, \infty}$ normal vector on $\Gamma_{\mathrm{g}}$ and assume we have a $W^{1, \infty}$ diffeomorphism $\boldsymbol{\Phi}: \Gamma_{\mathrm{g}, \mathrm{h}} \rightarrow \Gamma_{\mathrm{g}}$ such that $\left\|\boldsymbol{\nu} \circ \mathbf{\Phi}-\boldsymbol{\nu}_{h}\right\|_{L^{\infty}\left(\Gamma_{\mathrm{g}, \mathrm{h}}\right)} \leq c_{0} h$. As was done in Propositions 3.3 and 3.4, one can show there exists a $\hat{\mathbf{v}}$ in $H^{1}\left(\Omega_{h}\right)$, with $\hat{\mathbf{v}} \cdot \mathbf{e}_{y}=0$ on $\Gamma_{\mathrm{s}, \mathrm{h}}$, such that

$$
\int_{\Gamma_{\mathrm{g}, \mathrm{h}}} \mu_{h}(\hat{\mathbf{v}} \cdot(\boldsymbol{\nu} \circ \mathbf{\Phi}))=\left\|\mu_{h}\right\|_{\mathbb{M}}, \quad\|\hat{\mathbf{v}}\|_{H^{1}\left(\Omega_{h}\right)}=1 .
$$

Let $\hat{\mathbf{v}}_{h}:=\Pi_{h} \hat{\mathbf{v}} \in \mathbb{V}_{h}$ be the Scott-Zhang interpolant onto $\mathbb{V}_{h} ;$ thus

$$
\left\|\hat{\mathbf{v}}_{h}\right\|_{H^{1}\left(\Omega_{h}\right)} \leq c_{1}, \quad\left\|\hat{\mathbf{v}}_{h}-\hat{\mathbf{v}}\right\|_{L^{2}\left(\Gamma_{\mathrm{g}, \mathrm{h}}\right)} \leq c_{2} h^{1 / 2}\|\hat{\mathbf{v}}\|_{H^{1 / 2}\left(\Gamma_{\mathrm{g}, \mathrm{h}}\right)}=c_{3} h^{1 / 2} .
$$

Plugging into the discrete boundary integral form, we get

$$
\begin{aligned}
\int_{\Gamma_{\mathrm{g}, \mathrm{h}}} \mu_{h}\left(\hat{\mathbf{v}}_{h} \cdot \boldsymbol{\nu}_{h}\right)= & \int_{\Gamma_{\mathrm{g}, \mathrm{h}}} \mu_{h}(\hat{\mathbf{v}} \cdot(\boldsymbol{\nu} \circ \boldsymbol{\Phi}))+\int_{\Gamma_{\mathrm{g}, \mathrm{h}}} \mu_{h}\left(\hat{\mathbf{v}}_{h}-\hat{\mathbf{v}}\right) \cdot(\boldsymbol{\nu} \circ \mathbf{\Phi}) \\
& +\int_{\Gamma_{\mathrm{g}, \mathrm{h}}} \mu_{h}\left(\hat{\mathbf{v}}_{h} \cdot\left(\boldsymbol{\nu}_{h}-\boldsymbol{\nu} \circ \mathbf{\Phi}\right)\right) \\
\geq & \left\|\mu_{h}\right\|_{\mathbb{M}}-\left\|\mu_{h}\right\|_{L^{2}\left(\Gamma_{\mathrm{g}, \mathrm{h}}\right)}\left\|\hat{\mathbf{v}}_{h}-\hat{\mathbf{v}}\right\|_{L^{2}\left(\Gamma_{\mathrm{g}, \mathrm{h}}\right)} \\
& -\left\|\mu_{h}\right\|_{L^{2}\left(\Gamma_{\mathrm{g}, \mathrm{h}}\right)}\left\|\hat{\mathbf{v}}_{h}\right\|_{L^{2}\left(\Gamma_{\mathrm{g}, \mathrm{h}}\right)}\left\|\boldsymbol{\nu}_{h}-\boldsymbol{\nu} \circ \boldsymbol{\Phi}\right\|_{L^{\infty}\left(\Gamma_{\mathrm{g}, \mathrm{h}}\right)} \\
\geq & \left\|\mu_{h}\right\|_{\mathbb{M}}-c_{3} h^{1 / 2}\left\|\mu_{h}\right\|_{L^{2}\left(\Gamma_{\mathrm{g}, \mathrm{h}}\right)}-c_{4} h\left\|\mu_{h}\right\|_{L^{2}\left(\Gamma_{\mathrm{g}, \mathrm{h}}\right)} \\
\geq & \left\|\mu_{h}\right\|_{\mathbb{M}}-c_{5} h^{1 / 2}\left\|\mu_{h}\right\|_{L^{2}\left(\Gamma_{\mathrm{g}, \mathrm{h}}\right)}
\end{aligned}
$$

where we used (4.27), the Cauchy-Schwarz inequality, and previous bounds. Note, we must restrict $h<1$ to guarantee (4.28).

Now combine the discrete vector fields: $\mathbf{z}_{h}=\frac{c_{5}}{C} \mathbf{u}_{h}+\hat{\mathbf{v}}_{h}$. Then, $\left\|\mathbf{z}_{h}\right\|_{H^{1}\left(\Omega_{h}\right)} \leq \frac{c_{5}}{C}+c_{1}$ and

$$
\begin{aligned}
\int_{\Gamma_{\mathrm{g}, \mathrm{h}}} \mu_{h}\left(\mathbf{z}_{h} \cdot \boldsymbol{\nu}_{h}\right) & =\frac{c_{5}}{C} \int_{\Gamma_{\mathrm{g}, \mathrm{h}}} \mu_{h}\left(\mathbf{u}_{h} \cdot \boldsymbol{\nu}_{h}\right)+\int_{\Gamma_{\mathrm{g}, \mathrm{h}}} \mu_{h}\left(\hat{\mathbf{v}}_{h} \cdot \boldsymbol{\nu}_{h}\right) \\
& \geq c_{5} h^{1 / 2}\left\|\mu_{h}\right\|_{L^{2}\left(\Gamma_{\mathrm{g}, \mathrm{h}}\right)}+\left\|\mu_{h}\right\|_{\mathbb{M}}-c_{5} h^{1 / 2}\left\|\mu_{h}\right\|_{L^{2}\left(\Gamma_{\mathrm{g}, \mathrm{h}}\right)}=\left\|\mu_{h}\right\|_{\mathbb{M} \mathbf{}} .
\end{aligned}
$$

Therefore, we obtain the inf-sup condition:

$$
\sup _{\mathbf{z}_{h} \in \mathbb{V}_{h}} \frac{\int_{\Gamma_{\mathrm{g}, \mathrm{h}}} \mu_{h}\left(\mathbf{z}_{h} \cdot \boldsymbol{\nu}_{h}\right)}{\left\|\mathbf{z}_{h}\right\|_{H^{1}\left(\Omega_{h}\right)}} \geq \beta_{2}\left\|\mu_{h}\right\|_{\mathbb{M},}, \quad \text { where } \quad \beta_{2}=\frac{1}{\frac{c_{5}}{C}+c_{1}} .
$$


We need one more lemma to enable the proof of the full discrete inf-sup condition.

Lemma 4.10. Assume the hypothesis of Proposition 4.6. Let $\mu_{h}$ be in $\mathbb{M}_{h}$, where $\mathbb{M}_{h}=M_{0}$ or $\mathbb{M}_{h}=M_{1}$, and $\xi_{L}, \xi_{R}$ in $\mathbb{R}$. Then there is a constant $\zeta_{0}>0$ (depending only on $\Gamma_{\mathrm{g}}$ ), such that we have the following results.

1. There exists a $\mathbf{Y}_{h, 0}$ in $\mathbb{Y}_{h}$ such that

$$
\begin{gathered}
\mathbf{Y}_{h, 0}=\mathbf{0}, \text { on } \partial \Gamma_{\mathrm{g}, \mathrm{h}}, \quad-\int_{\Gamma_{\mathrm{g}, \mathrm{h}}} \mu_{h}\left(\mathbf{Y}_{h, 0} \cdot \boldsymbol{\nu}_{h}\right) \geq\left\|\mu_{h}\right\|_{H^{-1}\left(\Gamma_{\mathrm{g}, \mathrm{h}}\right)}+\zeta_{0} h\left\|\mu_{h}\right\|_{L^{2}\left(\Gamma_{\mathrm{g}, \mathrm{h}}\right)}, \\
\left\|\mathbf{Y}_{h, 0}\right\|_{H^{1}\left(\Gamma_{\mathrm{g}, \mathrm{h}}\right)} \leq C_{0},
\end{gathered}
$$

where $C_{0}$ depends on $\widetilde{\boldsymbol{\nu}}$ in Lemma 3.2 and the geometry of $\Gamma_{\mathrm{g}}$.

2. There exists a $\mathbf{Y}_{h, 1}$ in $\mathbb{Y}_{h}$ such that

$$
\begin{aligned}
&\left.\mathbf{Y}_{h, 1}\right|_{\mathbf{x}_{\mathrm{cl}}^{L}}=-\operatorname{sgn}\left(\xi_{L}\right) \frac{\mathbf{e}_{x}}{\mathbf{e}_{x} \cdot \boldsymbol{\nu}_{h}},\left.\quad \mathbf{Y}_{h, 1}\right|_{\mathbf{x}_{\mathrm{cl}}^{R}}=\operatorname{sgn}\left(\xi_{R}\right) \frac{\mathbf{e}_{x}}{\mathbf{e}_{x} \cdot \boldsymbol{\nu}_{h}}, \\
& \frac{\left|\xi_{L}\right|}{\alpha_{0}} \geq\left.\xi_{L}\left(\mathbf{Y}_{h, 1} \cdot \mathbf{e}_{x}\right)\right|_{\mathbf{x}_{\mathrm{cl}}^{L}} \geq\left|\xi_{L}\right|, \quad \frac{\left|\xi_{R}\right|}{\alpha_{0}} \geq\left.\xi_{R}\left(\mathbf{Y}_{h, 1} \cdot \mathbf{e}_{x}\right)\right|_{\mathbf{x}_{\mathrm{cl}}^{R}} \geq\left|\xi_{R}\right|, \\
&\left|\int_{\Gamma_{\mathrm{g}, \mathrm{h}}} \mu_{h}\left(\mathbf{Y}_{h, 1} \cdot \boldsymbol{\nu}_{h}\right)\right| \leq \zeta_{0} h\left\|\mu_{h}\right\|_{L^{2}\left(\Gamma_{\mathrm{g}, \mathrm{h}}\right)}, \quad\left\|\mathbf{Y}_{h, 1}\right\|_{H^{1}\left(\Gamma_{\mathrm{g}, \mathrm{h}}\right)} \leq C_{1},
\end{aligned}
$$

where $\alpha_{0}$ is taken from Lemma 3.2, and $C_{1}$ depends on $\widetilde{\boldsymbol{\nu}}$ and $\alpha_{0}^{-1}$.

Proof. First note that, similar to the proof of Lemma 3.11, one can show there is a $\mathbf{Y}_{0}$ in $H^{1}\left(\Gamma_{\mathrm{g}, \mathrm{h}}\right)$ such that

$$
\mathbf{Y}_{0}=\mathbf{0}, \text { on } \partial \Gamma_{\mathrm{g}, \mathrm{h}}, \quad-\int_{\Gamma_{\mathrm{g}, \mathrm{h}}} \mu_{h}\left(\mathbf{Y}_{0} \cdot(\boldsymbol{\nu} \circ \mathbf{\Phi})\right)=\left\|\mu_{h}\right\|_{H^{-1}\left(\Gamma_{\mathrm{g}, \mathrm{h}}\right)}, \quad\left\|\mathbf{Y}_{0}\right\|_{H^{1}\left(\Gamma_{\mathrm{g}, \mathrm{h}}\right)} \leq c_{0},
$$

where $\boldsymbol{\nu}$ and $\boldsymbol{\Phi}$ are defined as in the proof of Lemma 4.9. Let $\widehat{\mathbf{Y}}_{h, 0}$ in $\mathbb{Y}_{h}$ be the piecewise quadratic interpolant of $\mathbf{Y}_{0}$ over $\Gamma_{\mathrm{g}, \mathrm{h}}$. Then, by a similar argument as was shown in (4.28), we get

$$
-\int_{\Gamma_{\mathrm{g}, \mathrm{h}}} \mu_{h}\left(\widehat{\mathbf{Y}}_{h, 0} \cdot \boldsymbol{\nu}_{h}\right) \geq\left\|\mu_{h}\right\|_{H^{-1}\left(\Gamma_{\mathrm{g}, \mathrm{h}}\right)}-c_{1} h\left\|\mu_{h}\right\|_{L^{2}\left(\Gamma_{\mathrm{g}, \mathrm{h}}\right)}, \quad\left\|\widehat{\mathbf{Y}}_{h, 0}\right\|_{H^{1}\left(\Gamma_{\mathrm{g}, \mathrm{h}}\right)} \leq c_{2}
$$

Now use Proposition 4.8 to obtain a $\widetilde{\mathbf{Y}}_{h, 0}$ that satisfies

$$
-\int_{\Gamma_{\mathrm{g}, \mathrm{h}}} \mu_{h}\left(\widetilde{\mathbf{Y}}_{h, 0} \cdot \boldsymbol{\nu}_{h}\right) \geq c_{3} h\left\|\mu_{h}\right\|_{L^{2}\left(\Gamma_{\mathrm{g}, \mathrm{h}}\right)}, \quad\left\|\widetilde{\mathbf{Y}}_{h, 0}\right\|_{H^{1}\left(\Gamma_{\mathrm{g}, \mathrm{h}}\right)}=1
$$

and define $\mathbf{Y}_{h, 0}=\widehat{\mathbf{Y}}_{h, 0}+\left(\frac{c_{1}+\zeta_{0}}{c_{3}}\right) \widetilde{\mathbf{Y}}_{h, 0}$, where $\zeta_{0}>0$ is yet to be specified. Then it is clear that $\mathbf{Y}_{h, 0}$ satisfies (4.29).

Next, interpolate the (modified) function $\mathbf{Y}_{1}$ over $\Gamma_{\mathrm{g}, \mathrm{h}}$ from Lemma 3.11, i.e. let $\widehat{\mathbf{Y}}_{h, 1}$ in $\mathbb{Y}_{h}$ be the piecewise quadratic interpolant of $\mathbf{Y}_{1}$ and proceed as before. The constant $\zeta_{0}$ comes out of that.

Proof of Theorem 4.5. Let $q_{h} \in \mathbb{Q}_{h}, \mu_{h} \in \mathbb{M}_{h}$, and $\xi_{L}, \xi_{R} \in \mathbb{R}$ be arbitrary. Starting as we did in Theorem 3.12, we have

$$
\begin{aligned}
b_{h}\left(\left(\mathbf{v}_{h}, \mathbf{Y}_{h}\right),\left(q_{h}, \mu_{h}, \xi_{L}, \xi_{R}\right)\right)= & -\int_{\Omega_{h}} \tilde{q}_{h} \nabla \cdot \mathbf{v}_{h}+\int_{\Gamma_{\mathrm{g}, \mathrm{h}}}\left(\mu_{h}-q_{h, 0}\right) \mathbf{v}_{h} \cdot \boldsymbol{\nu}_{h}-\frac{1}{\delta t} \int_{\Gamma_{\mathrm{g}, \mathrm{h}}} \mu_{h}\left(\mathbf{Y}_{h} \cdot \boldsymbol{\nu}_{h}\right) \\
& +\left.\frac{1}{\delta t} \xi_{L}\left(\mathbf{Y}_{h} \cdot \mathbf{e}_{x}\right)\right|_{\mathbf{x}_{\mathrm{cl}}^{L}}+\left.\frac{1}{\delta t} \xi_{R}\left(\mathbf{Y}_{h} \cdot \mathbf{e}_{x}\right)\right|_{\mathbf{x}_{\mathrm{cl}}^{R}}
\end{aligned}
$$


where $q_{h}=\tilde{q}_{h}+q_{h, 0}$ and $q_{h, 0}=\frac{1}{\left|\Omega_{h}\right|} \int_{\Omega_{h}} q_{h}$. By Lemma 4.9, there exists a $\hat{\mathbf{u}}_{h}$ in $\mathbb{V}_{h}$ such that

$$
\int_{\Gamma_{\mathrm{g}, \mathrm{h}}}\left(\mu_{h}-q_{h, 0}\right) \hat{\mathbf{u}}_{h} \cdot \boldsymbol{\nu}_{h} \geq \beta_{2}\left\|\mu_{h}-q_{h, 0}\right\|_{\mathbb{M}}, \quad\left\|\hat{\mathbf{u}}_{h}\right\|_{H^{1}\left(\Omega_{h}\right)}=1 .
$$

Next, consider the discrete Stokes problem, which has a unique solution $\left(\mathbf{u}_{h}^{*}, p_{h}^{*}\right) \in \mathbb{V}_{h, 0} \times \mathbb{Q}_{h, 0}[11,13]$,

$$
\begin{aligned}
\int_{\Omega_{h}} \nabla \mathbf{u}_{h}^{*}: \nabla \mathbf{v}_{h} & -\int_{\Omega_{h}} p_{h}^{*} \nabla \cdot \mathbf{v}_{h}=0 \\
& -\int_{\Omega_{h}} \rho_{h} \nabla \cdot \mathbf{u}_{h}^{*}=\int_{\Omega_{h}} \rho_{h}\left(\frac{\tilde{q}_{h}}{\left\|\tilde{q}_{h}\right\|_{L^{2}\left(\Omega_{h}\right)}}+\nabla \cdot \hat{\mathbf{u}}_{h}\right),
\end{aligned}
$$

for all $\mathbf{v}_{h} \in \mathbb{V}_{h, 0}$ and $\rho_{h} \in \mathbb{Q}_{h, 0}$, where $\mathbb{V}_{h, 0}=\mathbb{V}_{h} \cap H_{0}^{1}\left(\Omega_{h}\right)$.

By (4.35), we have

$$
\begin{aligned}
\left|\mathbf{u}_{h}^{*}\right|_{H^{1}\left(\Omega_{h}\right)}^{2}=\int_{\Omega_{h}} p_{h}^{*} \nabla \cdot \mathbf{u}_{h}^{*} & =-\left(\int_{\Omega_{h}} \frac{p_{h}^{*} \tilde{q}_{h}}{\left\|\tilde{q}_{h}\right\|_{L^{2}\left(\Omega_{h}\right)}}+\int_{\Omega_{h}} p_{h}^{*} \nabla \cdot \hat{\mathbf{u}}_{h}\right) \\
& \leq\left\|p_{h}^{*}\right\|_{L^{2}\left(\Omega_{h}\right)}+\left\|p_{h}^{*}\right\|_{L^{2}\left(\Omega_{h}\right)}\left\|\nabla \cdot \hat{\mathbf{u}}_{h}\right\|_{L^{2}\left(\Omega_{h}\right)} \leq 2\left\|p_{h}^{*}\right\|_{L^{2}\left(\Omega_{h}\right)},
\end{aligned}
$$

using (4.34) and the fact that $\left\|\nabla \cdot \hat{\mathbf{u}}_{h}\right\|_{L^{2}\left(\Omega_{h}\right)} \leq\left\|\hat{\mathbf{u}}_{h}\right\|_{H^{1}\left(\Omega_{h}\right)}=1$. We also have, by using the inf-sup condition for the discrete Stokes problem [13], the following bound:

$$
\tilde{\beta}\left\|p_{h}^{*}\right\|_{L^{2}\left(\Omega_{h}\right)} \leq \sup _{\mathbf{v}_{h} \in \mathbb{V}_{h, 0}} \frac{\int_{\Omega_{h}} p_{h}^{*} \nabla \cdot \mathbf{v}_{h}}{\left\|\mathbf{v}_{h}\right\|_{H^{1}\left(\Omega_{h}\right)}}=\sup _{\mathbf{v}_{h} \in \mathbb{V}_{h, 0}} \frac{\int_{\Omega_{h}} \nabla \mathbf{u}_{h}^{*}: \nabla \mathbf{v}_{h}}{\left\|\mathbf{v}_{h}\right\|_{H^{1}\left(\Omega_{h}\right)}}=\left\|\mathbf{u}_{h}^{*}\right\|_{H^{1}\left(\Omega_{h}\right)} .
$$

Hence, $\left\|p_{h}^{*}\right\|_{L^{2}\left(\Omega_{h}\right)} \leq \frac{1}{\tilde{\beta}}\left\|\mathbf{u}_{h}^{*}\right\|_{H^{1}\left(\Omega_{h}\right)}$. Combining with (4.36), we have $\left\|\mathbf{u}_{h}^{*}\right\|_{H^{1}\left(\Omega_{h}\right)} \leq \frac{2 c_{1}}{\tilde{\beta}}=: c_{2}$, because $\mathbf{u}_{h}^{*}$ has zero boundary data.

Next, let $\mathbf{u}_{h}:=\hat{\mathbf{u}}_{h}+\mathbf{u}_{h}^{*}$. By the previous steps, we know that $\left\|\mathbf{u}_{h}\right\|_{H^{1}\left(\Omega_{h}\right)} \leq 1+c_{2}$, and using (4.35), we get the following inequality:

$$
\begin{aligned}
-\int_{\Omega_{h}} \tilde{q}_{h} \nabla \cdot \mathbf{u}_{h}+\int_{\Gamma_{\mathrm{g}, \mathrm{h}}}\left(\mu_{h}-q_{h, 0}\right) \mathbf{u}_{h} \cdot \boldsymbol{\nu}_{h} & =-\int_{\Omega_{h}} \tilde{q}_{h}\left(\nabla \cdot \hat{\mathbf{u}}_{h}+\nabla \cdot \mathbf{u}_{h}^{*}\right)+\int_{\Gamma_{\mathrm{g}, \mathrm{h}}}\left(\mu_{h}-q_{h, 0}\right) \hat{\mathbf{u}}_{h} \cdot \boldsymbol{\nu}_{h} \\
& \geq\left\|\tilde{q}_{h}\right\|_{L^{2}\left(\Omega_{h}\right)}+\beta_{2}\left\|\mu_{h}-q_{h, 0}\right\|_{\mathbb{M}},
\end{aligned}
$$

which addresses part of the inf-sup condition.

Now apply Lemma 4.10 to construct $\mathbf{W}_{h}=\mathbf{Y}_{h, 0}+\mathbf{Y}_{h, 1}$ in $\mathbb{Y}_{h}$ (which depends on $\mu_{h}, \xi_{L}, \xi_{R}$ ). Insert $\mathbf{u}_{h}$ and $\mathbf{W}_{h}$ into (4.33):

$$
\begin{gathered}
b_{h}\left(\left(\mathbf{u}_{h}, \mathbf{W}_{h}\right),\left(q_{h}, \mu_{h}, \xi_{L}, \xi_{R}\right)\right) \geq\left\|\tilde{q}_{h}\right\|_{L^{2}\left(\Omega_{h}\right)}+\beta_{2}\left\|\mu_{h}-q_{h, 0}\right\|_{\mathbb{M}}-\frac{1}{\delta t} \int_{\Gamma_{\mathrm{g}, \mathrm{h}}} \mu_{h}\left(\mathbf{W}_{h} \cdot \boldsymbol{\nu}_{h}\right) \\
\quad+\left.\frac{1}{\delta t} \xi_{L}\left(\mathbf{W}_{h} \cdot \mathbf{e}_{x}\right)\right|_{\mathbf{x}_{\mathrm{cl}}^{L}}+\left.\frac{1}{\delta t} \xi_{R}\left(\mathbf{W}_{h} \cdot \mathbf{e}_{x}\right)\right|_{\mathbf{x}_{\mathrm{cl}}^{R}}, \\
\geq\left\|\tilde{q}_{h}\right\|_{L^{2}\left(\Omega_{h}\right)}+\beta_{2}\left\|\mu_{h}-q_{h, 0}\right\|_{\mathbb{M}}+\frac{1}{\delta t}\left(\left\|\mu_{h}\right\|_{H^{-1}\left(\Gamma_{\mathrm{g}, \mathrm{h}}\right)}+\left|\xi_{L}\right|+\left|\xi_{R}\right|\right) \\
\geq \frac{c_{3}}{\delta t}\left\|\left(q_{h}, \mu_{h}, \xi_{L}, \xi_{R}\right)\right\|_{\mathbb{T}},
\end{gathered}
$$

where we used (3.18). By the bound on $\mathbf{u}_{h}$ and Lemma 4.10, we have the bound

$$
\left\|\left(\mathbf{u}_{h}, \mathbf{W}_{h}\right)\right\|_{\mathbb{Z}} \leq c_{4},
$$

where $c_{4}$ depends on $\Omega$ and $\alpha_{0}^{-1}$. Finally, we form the ratio in (4.17), use (4.42), take the supremum, and noting that $q_{h}, \mu_{h}, \xi_{L}$, and $\xi_{R}$ are arbitrary, the assertion follows with $\beta:=\frac{c_{3}}{\delta t \cdot c_{4}}$. 


\subsection{Fully discrete energy law}

The fully discrete formulation possesses an energy law.

Proposition 4.11. Assume the hypothesis of Proposition 3.14. For all $0 \leq n \leq N-1$, suppose $\delta t=t_{n+1}-t_{n}$ is uniform and the solution of (4.10) satisfies: $\Gamma_{\mathrm{g}, \mathrm{h}}^{n}$ is $W^{1, \infty}$ and is parameterized by $\mathbf{X}_{h}^{n}$, and $\Gamma_{\mathrm{s}}^{n}$ is flat with positive measure. Then,

$$
\delta t \sum_{i=0}^{N-1}\left[\left\|\mathbf{u}_{h}^{i+1}\right\|_{H^{1}\left(\Omega_{h}^{i}\right)}^{2}+\left.\left(\mathbf{V}_{h}^{i+1} \cdot \mathbf{e}_{x}\right)^{2}\right|_{\mathbf{x}_{\mathrm{cl}}}\right]+\left|\Gamma_{\mathrm{g}, \mathrm{h}}^{N}\right| \leq C\left\{\left|\Gamma_{\mathrm{g}, \mathrm{h}}^{0}\right|+\delta t \sum_{i=0}^{N-1}\left[\left\|\mathbf{f}^{i+1}\right\|_{L^{2}\left(\Omega_{h}^{i}\right)}^{2}+\left.\left(\gamma_{\mathrm{s}}^{i+1}-\gamma_{\mathrm{s}, \mathrm{g}}^{i+1}\right)^{2}\right|_{\mathbf{x}_{\mathrm{cl}}}\right]\right\},
$$

where $\mathbf{V}_{h}^{i+1}=\left(\mathbf{X}_{h}^{i+1}-\mathrm{id}_{\Gamma_{\mathrm{g}, \mathrm{h}}^{i}}\right) / \delta$ t and the constant $C$ only depends on the domain.

Proof. Since the finite element spaces are conforming, the proof is the same as for Proposition 3.14.

\section{ERror estimates}

We derive preliminary error estimates for the fully discrete formulation (4.10) at a single time step. Throughout this section, we shall invoke the variational crime argument, and ignore the subscript $h$ in the bilinear and linear forms of the fully discrete problem $(4.10)$, i.e. $a_{h}(\cdot, \cdot) \equiv a(\cdot, \cdot)$, etc. In particular, we will not deal explicitly with the normal vector approximation (recall Rem. 4.2) in order to avoid additional technicalities.

\subsection{Error equations}

Lemma 5.1 is a special case of Lemma 2.7 in [63]; we omit the proof. Also, Lemma 5.2 is a modification of Lemma 2.9 in [63].

Lemma 5.1. Let $\left(\mathbf{u}, \mathbf{W}, p, \Lambda_{g}, \lambda_{L}, \lambda_{R}\right)$ solve the time semi-discrete problem (3.20) and $\left(\mathbf{u}_{h}, \mathbf{W}_{h}, p_{h}, \Lambda_{g, h}, \lambda_{L, h}, \lambda_{R, h}\right)$ solve the fully discrete problem (4.10). Then, for all $(\mathbf{v}, \mathbf{Y})$ in $\mathbb{Z},\left(\mathbf{v}_{h}, \mathbf{Y}_{h}\right)$ in $\mathbb{Z}_{h},\left(q, \mu, \xi_{L}, \xi_{R}\right)$ in $\mathbb{T}$, and $\left(q_{h}, \mu_{h}, \xi_{L, h}, \xi_{R, h}\right)$ in $\mathbb{T}_{h}$, the following inequality holds:

$$
\begin{aligned}
a\left(\left(\mathbf{v}_{h}-\mathbf{u}_{h}, \mathbf{Y}_{h}-\mathbf{W}_{h}\right),\right. & \left.\left(\mathbf{v}_{h}-\mathbf{u}_{h}, \mathbf{Y}_{h}-\mathbf{W}_{h}\right)\right) \leq B_{1}\left(\left(q_{h}, \mu_{h}, \xi_{L, h}, \xi_{R, h}\right)\right)+B_{2}\left(\left(q, \mu, \xi_{L}, \xi_{R}\right)\right) \\
& +a\left(\left(\mathbf{v}_{h}-\mathbf{u}, \mathbf{Y}_{h}-\mathbf{W}\right),\left(\mathbf{v}_{h}-\mathbf{u}_{h}, \mathbf{Y}_{h}-\mathbf{W}_{h}\right)\right) \\
& +b\left(\left(\mathbf{v}_{h}-\mathbf{u}, \mathbf{Y}_{h}-\mathbf{W}\right),\left(p_{h}-p, \Lambda_{g, h}-\Lambda_{g}, \lambda_{L, h}-\lambda_{L}, \lambda_{R, h}-\lambda_{R}\right)\right) \\
& +b\left(\left(\mathbf{u}_{h}-\mathbf{u}, \mathbf{W}_{h}-\mathbf{W}\right),\left(p-q_{h}, \Lambda_{g}-\mu_{h}, \lambda_{L}-\xi_{L, h}, \lambda_{R}-\xi_{R, h}\right)\right),
\end{aligned}
$$

where

$$
\begin{aligned}
B_{1}\left(\left(q_{h}, \mu_{h}, \xi_{L, h}, \xi_{R, h}\right)\right)= & b\left((\mathbf{u}, \mathbf{W}),\left(p-q_{h}, \Lambda_{g}-\mu_{h}, \lambda_{L}-\xi_{L, h}, \lambda_{R}-\xi_{R, h}\right)\right) \\
& -G\left(\left(p-q_{h}, \Lambda_{g}-\mu_{h}, \lambda_{L}-\xi_{L, h}, \lambda_{R}-\xi_{R, h}\right)\right), \\
B_{2}\left(\left(q, \mu, \xi_{L}, \xi_{R}\right)\right)= & b\left((\mathbf{u}, \mathbf{W}),\left(p_{h}-q, \Lambda_{g, h}-\mu, \lambda_{L, h}-\xi_{L}, \lambda_{R, h}-\xi_{R}\right)\right) \\
& -G\left(\left(p_{h}-q, \Lambda_{g, h}-\mu, \lambda_{L, h}-\xi_{L}, \lambda_{R, h}-\xi_{R}\right)\right) .
\end{aligned}
$$

Lemma 5.2. Assume the hypothesis of Lemma 5.1. Let $\Gamma_{\mathrm{g}}$ be of class $W^{2, \infty}$ and assume $h$ is sufficiently small to ensure the inf-sup condition in Theorem 4.5. Then the following inequalities hold:

$$
\begin{aligned}
\left\|\left(p-p_{h}, \Lambda_{g}-\Lambda_{g, h}, \lambda_{L}-\lambda_{L, h}, \lambda_{R}-\lambda_{R, h}\right)\right\|_{\mathbb{T}} \leq & C_{1}\left(\left\|\left(q_{h}-p, \mu_{h}-\Lambda_{g}, \xi_{L, h}-\lambda_{L}, \xi_{R, h}-\lambda_{R}\right)\right\|_{\mathbb{T}}\right. \\
& \left.+\left\|\left(\mathbf{u}-\mathbf{u}_{h}, \mathbf{W}-\mathbf{W}_{h}\right)\right\|_{\mathbb{Z}}\right), \\
\left\|\left(\mathbf{u}-\mathbf{u}_{h}, \mathbf{W}-\mathbf{W}_{h}\right)\right\|_{\mathbb{Z}}^{2} \leq & C_{2}\left(B_{1}\left(\left(q_{h}, \mu_{h}, \xi_{L, h}, \xi_{R, h}\right)\right)+\right. \\
+ & B_{2}\left(\left(q, \mu, \xi_{L}, \xi_{R}\right)\right) \\
& \left.+\left\|\left(p-q_{h}, \Lambda_{g}-\mu_{h}, \lambda_{L}-\xi_{L, h}, \lambda_{R}-\xi_{R, h}\right)\right\|_{\mathbb{T}}^{2}+\left\|\left(\mathbf{u}-\mathbf{v}_{h}, \mathbf{W}-\mathbf{Y}_{h}\right)\right\|_{\mathbb{Z}}^{2}\right),
\end{aligned}
$$


for all $\left(\mathbf{v}_{h}, \mathbf{Y}_{h}\right)$ in $\mathbb{Z}_{h},\left(q_{h}, \mu_{h}, \xi_{L, h}, \xi_{R, h}\right)$ in $\mathbb{T}_{h}$, and all $h$ sufficiently small. Note that $C_{1}$ and $C_{2}$ depend on $\delta t^{-1}$.

Proof. Using the exact and discrete solutions from (3.20) and (4.10), we get

$$
\begin{aligned}
b\left(\left(\mathbf{v}_{h}, \mathbf{Y}_{h}\right),\left(q_{h}\right.\right. & \left.\left.-p_{h}, \mu_{h}-\Lambda_{g, h}, \xi_{L, h}-\lambda_{L, h}, \xi_{R, h}-\lambda_{R, h}\right)\right) \\
= & b\left(\left(\mathbf{v}_{h}, \mathbf{Y}_{h}\right),\left(q_{h}, \mu_{h}, \xi_{L, h}, \xi_{R, h}\right)\right)-b\left(\left(\mathbf{v}_{h}, \mathbf{Y}_{h}\right),\left(p_{h}, \Lambda_{g, h}, \lambda_{L, h}, \lambda_{R, h}\right)\right) \\
& +F\left(\left(\mathbf{v}_{h}, \mathbf{Y}_{h}\right)\right)-a\left((\mathbf{u}, \mathbf{W}),\left(\mathbf{v}_{h}, \mathbf{Y}_{h}\right)\right)-b\left(\left(\mathbf{v}_{h}, \mathbf{Y}_{h}\right),\left(p, \Lambda_{g}, \lambda_{L}, \lambda_{R}\right)\right) \\
= & b\left(\left(\mathbf{v}_{h}, \mathbf{Y}_{h}\right),\left(q_{h}-p, \mu_{h}-\Lambda_{g}, \xi_{L, h}-\lambda_{L}, \xi_{R, h}-\lambda_{R}\right)\right) \\
& +a\left(\left(\mathbf{u}_{h}-\mathbf{u}, \mathbf{W}_{h}-\mathbf{W}\right),\left(\mathbf{v}_{h}, \mathbf{Y}_{h}\right)\right),
\end{aligned}
$$

for all $\left(q_{h}, \mu_{h}, \xi_{L, h}, \xi_{R, h}\right)$ in $\mathbb{T}_{h}$. So combining the discrete inf-sup condition provided by Theorem 4.5 with (5.4) gives

$$
\begin{array}{r}
\left\|\left(q_{h}-p_{h}, \mu_{h}-\Lambda_{g, h}, \xi_{L, h}-\lambda_{L, h}, \xi_{R, h}-\lambda_{R, h}\right)\right\|_{\mathbb{T}} \leq c_{1}\left(\left\|\left(\mathbf{u}-\mathbf{u}_{h}, \mathbf{W}-\mathbf{W}_{h}\right)\right\|_{\mathbb{Z}}\right. \\
\left.+\left\|\left(q_{h}-p, \mu_{h}-\Lambda_{g}, \xi_{L, h}-\lambda_{L}, \xi_{R, h}-\lambda_{R}\right)\right\|_{\mathbb{T}}\right)
\end{array}
$$

Starting with the left-hand-side of (5.2) and using a triangle inequality gives the rest of (5.2).

As for (5.3), we start with Lemma 5.1. Using the coercivity of $a(\cdot, \cdot)$ (recall (3.28)), Cauchy-Schwarz, and a triangle inequality, we have

$$
\begin{aligned}
\|\left(\mathbf{v}_{h}-\mathbf{u}_{h},\right. & \left.\mathbf{Y}_{h}-\mathbf{W}_{h}\right) \|_{\mathbb{Z}}^{2} \leq c_{2}\left\{B_{1}\left(\left(q_{h}, \mu_{h}, \xi_{L, h}, \xi_{R, h}\right)\right)+B_{2}\left(\left(q, \mu, \xi_{L}, \xi_{R}\right)\right)\right. \\
& +\left\|\left(\mathbf{v}_{h}-\mathbf{u}, \mathbf{Y}_{h}-\mathbf{W}\right)\right\|_{\mathbb{Z}}^{2}+\left\|\left(\mathbf{v}_{h}-\mathbf{u}, \mathbf{Y}_{h}-\mathbf{W}\right)\right\|_{\mathbb{Z}}\left\|\left(\mathbf{u}-\mathbf{u}_{h}, \mathbf{W}-\mathbf{W}_{h}\right)\right\|_{\mathbb{Z}} \\
& +\left\|\left(\mathbf{v}_{h}-\mathbf{u}, \mathbf{Y}_{h}-\mathbf{W}\right)\right\|_{\mathbb{Z}}\left\|\left(p_{h}-p, \Lambda_{g, h}-\Lambda_{g}, \lambda_{L, h}-\lambda_{L}, \lambda_{R, h}-\lambda_{R}\right)\right\|_{\mathbb{T}} \\
& \left.+\left\|\left(\mathbf{u}_{h}-\mathbf{u}, \mathbf{W}_{h}-\mathbf{W}\right)\right\|_{\mathbb{Z}}\left\|\left(p-q_{h}, \Lambda_{g}-\mu_{h}, \lambda_{L}-\xi_{L, h}, \lambda_{R}-\xi_{R, h}\right)\right\|_{\mathbb{T}}\right\} .
\end{aligned}
$$

Employing $\left\|\left(\mathbf{u}-\mathbf{u}_{h}, \mathbf{W}-\mathbf{W}_{h}\right)\right\|_{\mathbb{Z}}^{2} \leq 2\left\|\left(\mathbf{u}-\mathbf{v}_{h}, \mathbf{W}-\mathbf{Y}_{h}\right)\right\|_{\mathbb{Z}}^{2}+2\left\|\left(\mathbf{v}_{h}-\mathbf{u}_{h}, \mathbf{Y}_{h}-\mathbf{W}_{h}\right)\right\|_{\mathbb{Z}}^{2}$ and a weighted Young's inequality, we get the following inequality by combining with (5.6) and (5.2)

$$
\begin{aligned}
\left\|\left(\mathbf{u}-\mathbf{u}_{h}, \mathbf{W}-\mathbf{W}_{h}\right)\right\|_{\mathbb{Z}}^{2} \leq & c_{3}\left\{\left\|\left(\mathbf{u}-\mathbf{v}_{h}, \mathbf{W}-\mathbf{Y}_{h}\right)\right\|_{\mathbb{Z}}^{2}+B_{1}\left(\left(q_{h}, \mu_{h}, \xi_{L, h}, \xi_{R, h}\right)\right)+B_{2}\left(\left(q, \mu, \xi_{L}, \xi_{R}\right)\right)\right. \\
& \left.+\left\|\left(p-q_{h}, \Lambda_{g}-\mu_{h}, \lambda_{L}-\xi_{L, h}, \lambda_{R}-\xi_{R, h}\right)\right\|_{\mathbb{T}}^{2}\right\},
\end{aligned}
$$

which yields the estimate.

\subsection{Error estimate}

The forgoing results yield the following theorem.

Theorem 5.3 (Main error estimates). Assume the hypothesis of Lemma 5.2. Then we have the following error estimates for all $h$ sufficiently small:

$$
\begin{aligned}
\left\|\left(p-p_{h}, \Lambda_{g}-\Lambda_{g, h}, \lambda_{L}-\lambda_{L, h}, \lambda_{R}-\lambda_{R, h}\right)\right\|_{\mathbb{T}} & \leq C_{1}\left\{\left\|\left(\mathbf{u}-\mathbf{u}_{h}, \mathbf{W}-\mathbf{W}_{h}\right)\right\|_{\mathbb{Z}}\right. \\
& \left.+\inf _{\tilde{q}_{h} \in \mathbb{Q}_{h, 0}}\left\|\tilde{q}_{h}-\tilde{p}\right\|_{L^{2}\left(\Omega_{h}\right)}+\inf _{\mu_{h} \in \mathbb{M}_{h}}\left\|\mu_{h}-\Lambda_{g}\right\|_{\mathbb{M}}\right\},
\end{aligned}
$$

where $\sim$ denotes mean value zero and $\mathbb{Q}_{h, 0} \subset \mathbb{Q}_{h}$ is the space of discrete pressures with mean value zero,

$$
\begin{aligned}
\left\|\left(\mathbf{u}-\mathbf{u}_{h}, \mathbf{W}-\mathbf{W}_{h}\right)\right\|_{\mathbb{Z}}^{2} \leq & C_{2}\left\{\inf _{\mathbf{v}_{h} \in \mathbb{V}_{h}}\left\|\mathbf{u}-\mathbf{v}_{h}\right\|_{H^{1}\left(\Omega_{h}\right)}^{2}+\inf _{\mathbf{Y}_{h} \in \mathbb{Y}_{h}}\left\|\mathbf{W}-\mathbf{Y}_{h}\right\|_{H^{1}\left(\Gamma_{\mathrm{g}, \mathrm{h}}\right)}^{2}\right. \\
& \left.+\inf _{\tilde{q}_{h} \in \mathbb{Q}_{h, 0}}\left\|\tilde{q}_{h}-\tilde{p}\right\|_{L^{2}\left(\Omega_{h}\right)}^{2}+\inf _{\mu_{h} \in \mathbb{M}_{h}}\left\|\mu_{h}-\Lambda_{g}\right\|_{\mathbb{M}}^{2}\right\} .
\end{aligned}
$$


Proof. In Lemmas 5.1 and 5.2, we are free to choose the discrete test functions. Therefore, let $\xi_{L, h}=\lambda_{L}$ and $\xi_{R, h}=\lambda_{R}$, since they are just numbers and the convex set is the same in the semi-discrete and fully discrete cases. Moreover, set $q_{h}$ such that $q_{h, 0}:=\int_{\Omega_{h}} q_{h}=\int_{\Omega_{h}} p=: p_{0}$. Then, we have

$$
\begin{aligned}
\left\|\left(q_{h}-p, \mu_{h}-\Lambda_{g}, \xi_{L, h}-\lambda_{L}, \xi_{R, h}-\lambda_{R}\right)\right\|_{\mathbb{T}}^{2} & =\left\|\tilde{q}_{h}-\tilde{p}\right\|_{L^{2}\left(\Omega_{h}\right)}^{2}+\left\|\mu_{h}-\Lambda_{g}\right\|_{\mathbb{M}}^{2}+\left\|\mu_{h}-\Lambda_{g}\right\|_{H^{-1}\left(\Gamma_{\mathrm{g}, \mathrm{h}}\right)}^{2} \\
& \leq d_{0}\left\{\left\|\tilde{q}_{h}-\tilde{p}\right\|_{L^{2}\left(\Omega_{h}\right)}^{2}+\left\|\mu_{h}-\Lambda_{g}\right\|_{\mathbb{M}}^{2}\right\},
\end{aligned}
$$

where $\tilde{q}_{h}=q_{h}-q_{h, 0}$ and $\tilde{p}=p-p_{0}$. This yields (5.7).

Starting from (5.3), we find that $B_{2}$ vanishes by choosing the continuous test functions appropriately. Next, after making use of (3.20), we find that $B_{1}$ also vanishes, and so obtain (5.8).

The actual regularity of the solution $\left(\mathbf{u}, \mathbf{W}, p, \Lambda_{g}\right)$ of $(3.20)$ is open. However, because $\partial \Omega$ has corners with a change in boundary condition $\left(\Gamma_{\mathrm{g}}\right.$ to $\left.\Gamma_{\mathrm{s}}\right)$, the regularity of the Stokes equations is reduced from the standard Dirichlet case $[38,52]$. According to [52], the best regularity we can hope for the velocity and pressure is

$$
\mathbf{u} \text { in } H^{1+s}(\Omega), \quad p \text { in } H^{s}(\Omega), \quad \text { for } \frac{1}{2} \leq s \leq 1,
$$

where $s=\frac{1}{2}$ when $\theta_{\mathrm{cl}}=180^{\circ}-\epsilon$ and $s=1$ when $\theta_{\mathrm{cl}}=90^{\circ}-\epsilon$ (for $\epsilon>0$ small), with a continuous range in between. As $\theta_{\mathrm{cl}}$ decreases from $90^{\circ}$, the regularity further improves.

Because of the surface tension effect, the interface should be smooth; recall that, formally, $\Lambda_{g}$ is the curvature $\kappa$; see (2.22). Hence, it seems reasonable to assume the regularity of $\mathbf{W}$ and $\Lambda_{g}$ to be

$$
\mathbf{W} \text { in } H^{2+s}\left(\Gamma_{\mathrm{g}}\right), \quad \Lambda_{g} \text { in } H^{s}\left(\Gamma_{\mathrm{g}}\right), \quad \text { for } \frac{1}{2} \leq s \leq 1 .
$$

Therefore, using standard interpolation theory, for instance see [69], we obtain the following corollaries.

Corollary 5.4. Assume the hypothesis of Theorem 5.3 and assume the regularity in (5.9) and (5.10). Then we have

$$
\begin{aligned}
&\left\|\left(p-p_{h}, \Lambda_{g}-\Lambda_{g, h}, \lambda_{L}-\lambda_{L, h}, \lambda_{R}-\lambda_{R, h}\right)\right\|_{\mathbb{T}} \leq C_{1}\left\{\left\|\left(\mathbf{u}-\mathbf{u}_{h}, \mathbf{W}-\mathbf{W}_{h}\right)\right\|_{\mathbb{Z}}\right. \\
&\left.+h^{s}\|\tilde{p}\|_{H^{s}\left(\Omega_{h}\right)}+h^{s}\left\|\Lambda_{g}\right\|_{\left.H^{s}\left(\Gamma_{g, \mathrm{~h}}\right)\right\}}\right\}, \quad \text { for } \frac{1}{2} \leq s \leq 1, \\
&\left\|\left(\mathbf{u}-\mathbf{u}_{h}, \mathbf{W}-\mathbf{W}_{h}\right)\right\|_{\mathbb{Z}} \leq C_{2}\left\{h^{s}\|\mathbf{u}\|_{H^{1+s}\left(\Omega_{h}\right)}+h^{1+s}\|\mathbf{W}\|_{H^{2+s}\left(\Gamma_{\mathrm{g}, \mathrm{h}}\right)}\right. \\
&\left.+h^{s}\|\tilde{p}\|_{H^{s}\left(\Omega_{h}\right)}+h^{s}\left\|\Lambda_{g}\right\|_{\left.H^{s}\left(\Gamma_{\mathrm{g}, \mathrm{h}}\right)\right\}}\right\}, \quad \text { for } \frac{1}{2} \leq s \leq 1 .
\end{aligned}
$$

Note that $C_{1}, C_{2}$ depend on $\delta t^{-1}, \alpha_{0}^{-1}, \Omega$, and $\Gamma_{\mathrm{g}}$.

Proof. By [20], there exists Clément interpolation operators $\pi_{\mathbb{V}_{h}}, \pi_{\mathbb{Y}_{h}}, \pi_{\mathbb{Q}_{h}}, \pi_{\mathbb{M}_{h}}$ that satisfy:

$$
\begin{aligned}
\left\|\mathbf{u}-\pi_{\mathbb{V}_{h}} \mathbf{u}\right\|_{H^{1}(T)} & \leq c_{1} h^{l-1}\|\mathbf{u}\|_{H^{l}(T)}, & & 1 \leq l \leq 1+s, \\
\left\|\mathbf{W}-\pi_{\mathbb{Y}_{h}} \mathbf{W}\right\|_{H^{1}(E)} & \leq c_{2} h^{l-1}\|\mathbf{u}\|_{H^{l}(E)}, & & 1 \leq l \leq 2+s, \\
\left\|p-\pi_{\mathbb{Q}_{h}} p\right\|_{L^{2}(T)} & \leq c_{3} h^{l}\|\mathbf{u}\|_{H^{l}(T)}, & & 0 \leq l \leq s, \\
\left\|\Lambda_{g}-\pi_{\mathbb{M}_{h}} \Lambda_{g}\right\|_{L^{2}(E)} & \leq c_{4} h^{l}\left\|\Lambda_{g}\right\|_{H^{l}(E)}, & & 0 \leq l \leq s,
\end{aligned}
$$

where $T$ is in $\mathcal{T}_{h}$ and $E$ is in $\mathcal{E}_{h}$. Note that $\mathbb{M}_{h}=M_{0}$ or $\mathbb{M}_{h}=M_{1}$ and $\|\cdot\|_{\mathbb{M}} \leq \widetilde{C}\|\cdot\|_{L^{2}\left(\Gamma_{\mathrm{g}, \mathrm{h}}\right)}$. Thus, we obtain (5.11) and (5.12). 
Using iso-parametric elements allows us to reduce the consistency error due to the presence of the normal vector in the formulation [69]. Therefore, we obtain improved error estimates in $L^{2}$ norms for the primal variables by a classical duality argument in $[3,47]$ when using curved quadratic triangles.

Corollary 5.5. Assume the hypothesis of Theorem 5.3 and assume the regularity in (5.9) and (5.10). Furthermore, assume that $\Gamma_{\mathrm{g}}$ is $W^{3, \infty}$. Then we have

$$
\left\|\mathbf{u}-\mathbf{u}_{h}\right\|_{L^{2}\left(\Omega_{h}\right)}+\left\|\mathbf{W}-\mathbf{W}_{h}\right\|_{L^{2}\left(\Gamma_{\mathrm{g}, \mathrm{h}}\right)} \leq C_{3} h^{1+s}, \quad \text { for } \frac{1}{2} \leq s \leq 1 .
$$

Note that $C_{3}$ depends on $\delta t^{-1}, \alpha_{0}^{-1}, \Omega, \Gamma_{\mathrm{g}}$, and the exact solution of (3.20).

Proof. It is essentially the same argument as in [69].

\section{Numerical RESUlts}

\subsection{Setup}

\subsubsection{Navier-stokes}

In order to make the simulations more realistic, we change the Stokes momentum equation (2.24) to

$$
\operatorname{ReCa}\left[\partial_{t} \mathbf{u}+(\mathbf{u} \cdot \nabla) \mathbf{u}\right]-\nabla \cdot \boldsymbol{\sigma}=\operatorname{StCa} \mathbf{f}, \quad \text { on } \Omega .
$$

The time-discretization follows a standard ALE (Arbitrary-Lagrangian-Eulerian) [65] backward Euler method:

$$
\operatorname{ReCa}\left[\frac{\mathbf{u}^{n+1}-\mathbf{u}^{n}}{\delta t}+\left(\left(\mathbf{u}^{n}-\mathbf{c}^{n}\right) \cdot \nabla\right) \mathbf{u}^{n+1}\right]-\nabla \cdot \boldsymbol{\sigma}^{n+1}=\operatorname{StCa} \mathbf{f}^{n+1}
$$

where $\mathbf{c}^{n}$ is the mesh velocity at the previous time-step. An analysis of a full ALE method with generalized Navier boundary condition and surface tension, in the context of a geometric conservation law, is given in [36]. Indeed, it would be interesting to combine our analysis with that of [36].

\subsubsection{Solving the discrete system}

Solving the system (4.10) with inequality constraint is not difficult since the inequality is only active at two points. In fact, the following projection relation follows from (2.19):

$$
\lambda_{h}=\mathcal{P}_{\mathcal{K}}\left(\lambda_{h}+\varrho\left(\mathbf{W}_{h}-\mathbf{X}_{h}^{\text {old }}\right) \cdot \mathbf{e}_{x}\right), \quad \text { on } \mathbf{x}_{\mathrm{cl}}^{L} \text { and } \mathbf{x}_{\mathrm{cl}}^{R},
$$

where $\mathcal{P}_{\mathcal{K}}$ is the projection onto $\mathcal{K}, \lambda_{h} \equiv\left(\lambda_{L, h}, \lambda_{R, h}\right), \mathbf{W}_{h}$ is the discrete interface position solution, and $\varrho$ is any positive constant. All simulations were computed by using (6.3) to obtain the discrete solution at each time step; in addition, we used $\mathbb{M}_{h}=M_{1}$.

\subsection{Rolling droplet}

Figure 5 shows a simulation of a droplet rolling along a surface; parameters are given in Tables 2 and 3 . The non-dimensional body force has the form

$$
\mathbf{f}(t)=\min \left(\frac{t}{0.3}, 1\right) \text { St Ca } \mathbf{e}_{x} .
$$

Figure 6 shows the dynamics of the contact angles $\theta_{\mathrm{cl}}$ and contact points $\mathbf{x}_{\mathrm{cl}}$. The oscillations of the contact angles and contact point velocities are due to the presence of inertia in the fluid (i.e. non-zero Reynolds number). 

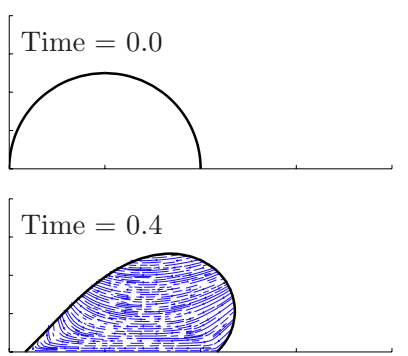
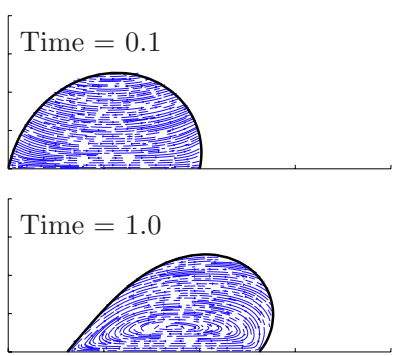
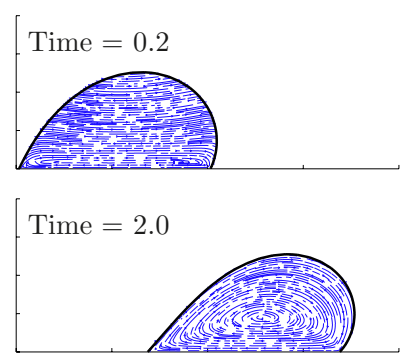

Figure 5. Simulation of a rolling droplet driven by a body force in the $\mathbf{e}_{x}$ direction. Plot window is $[0,2.0] \times[0,0.8]$ in non-dimensional units. Streamlines are plotted with respect to a frame of reference that moves with the droplet. The rolling motion in the last frame is in the clockwise direction. Times listed are in non-dimensional units.
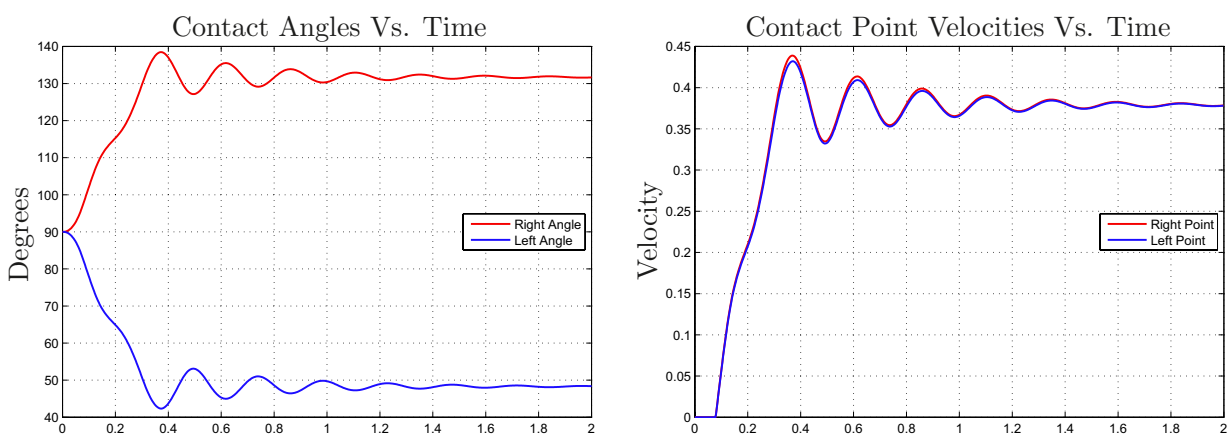

Figure 6. Dynamic contact angles and contact points for rolling droplet in Figure 5. The contact points have zero velocity near the beginning because of the pinning constraint. Time and velocity axes are in non-dimensional units.
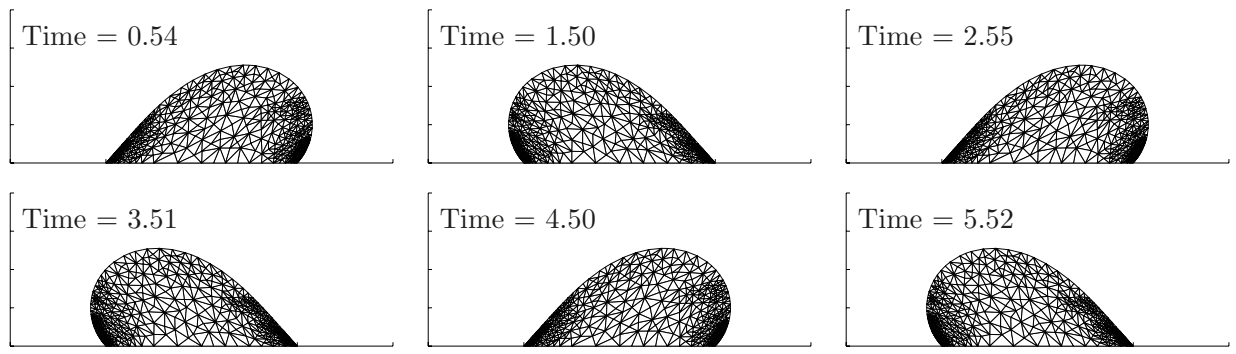

FigURE 7. Simulation of a wiggling droplet driven by a sinusoidal body force in the $\mathbf{e}_{x}$ direction. The triangulation of $\Omega_{h}$ is shown. Plot window is $[-0.5,1.5] \times[0,0.8]$ in non-dimensional units, and times listed are in non-dimensional units.

\subsection{Wiggling droplet}

Figure 7 shows a simulation of a droplet rocking back and forth on a surface. The parameters used are given in Tables 2 and 3 , except $C_{\text {pin }}$ is set very high to ensure the contact points are always pinned. The non-dimensional body force has the form

$$
\mathbf{f}(t)=\sin (\pi t) \operatorname{StCa} \mathbf{e}_{x} .
$$




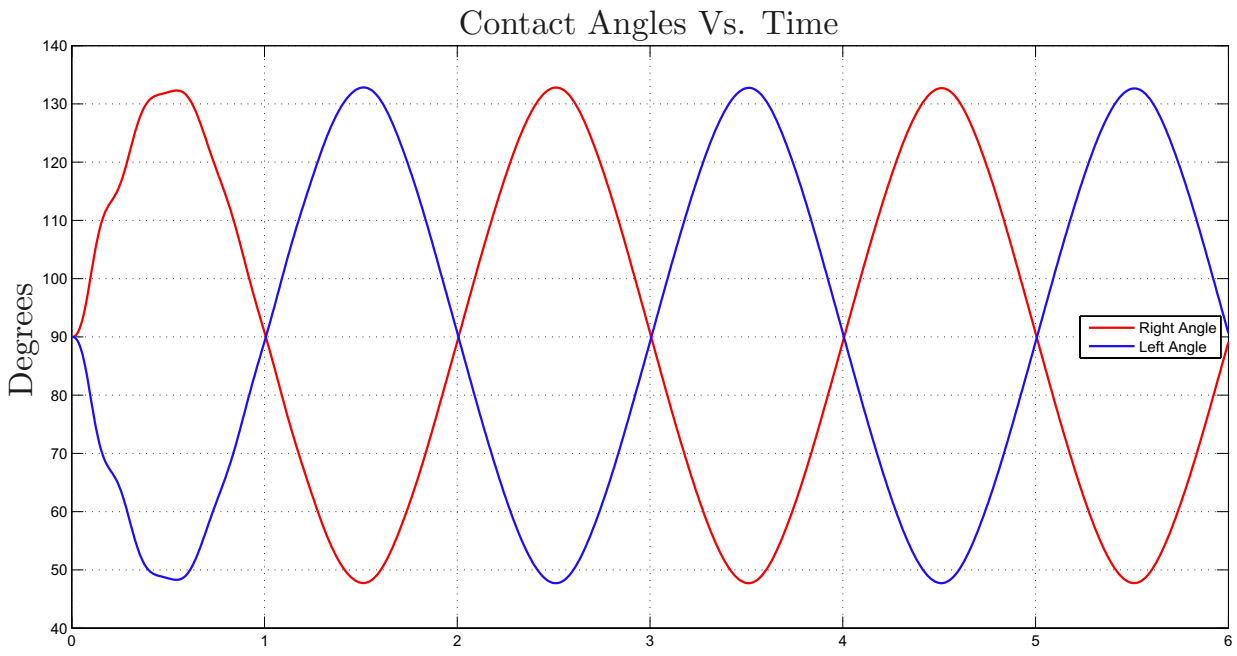

Figure 8. Dynamic contact angles for wiggling droplet in Figure 7. Note that the contact points are pinned throughout the simulation. Time axis is in non-dimensional units.

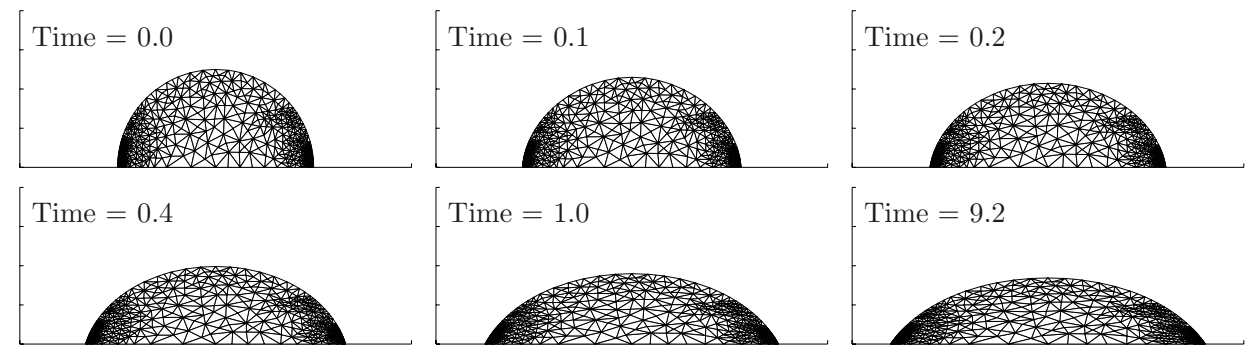

FIGURE 9. Simulation of a spreading droplet under gravity (wetting regime). The triangulation of $\Omega_{h}$ is shown. Plot window is $[-0.5,1.5] \times[0,0.8]$ in non-dimensional units, and times listed are in non-dimensional units.

Figure 8 shows the dynamics of the contact angles $\theta_{\mathrm{cl}}$. After an initial transient, the contact angles settle into a sinusoidal motion. Both contact angles go through equal deflections.

\subsection{Droplet spreading}

Figure 9 shows a simulation of a droplet spreading onto a surface. The parameters used are given in Tables 2 and 3, except $\gamma_{\mathrm{s}, \mathrm{g}}$ is changed to a value such that the equilibrium contact angle is $45^{\circ}$ and the non-dimensional body force is $\mathbf{f}=-\mathrm{StCa} \mathbf{e}_{y}$. Figure 10 shows the dynamics of the contact angles $\theta_{\mathrm{cl}}$ and contact points $\mathbf{x}_{\mathrm{cl}}$. Again, the oscillations of the contact angles and contact point velocities are due to the presence of inertia in the fluid. The droplet eventually reaches a stationary configuration with a contact angle of $55.375^{\circ}$, which is different from the equilibrium value because of the contact line pinning effect.

Figure 11 shows the correlation between contact angle and contact point velocity. The relationship appears to be roughly linear. A nonlinear relationship could be obtained by replacing the linear viscous contact line motion law $\left(\right.$ i.e. $\left.\beta_{\mathrm{cl}}\right)$ by something more nonlinear. 

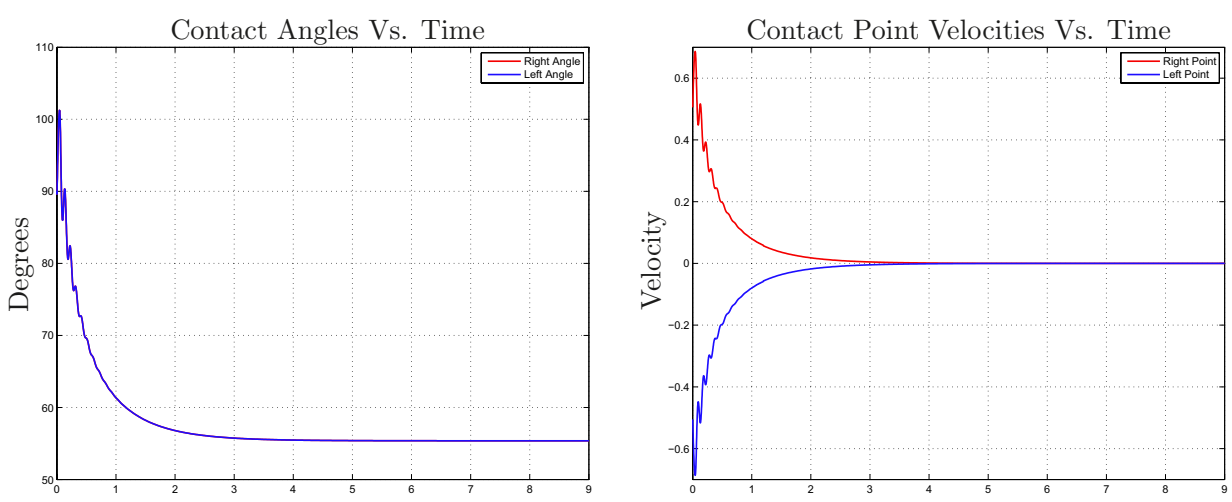

Figure 10. Dynamic contact angles and contact points for spreading droplet in Figure 9. The contact point velocities go to zero as the droplet approaches the equilibrium pinned configuration. Time and velocity axes are in non-dimensional units.
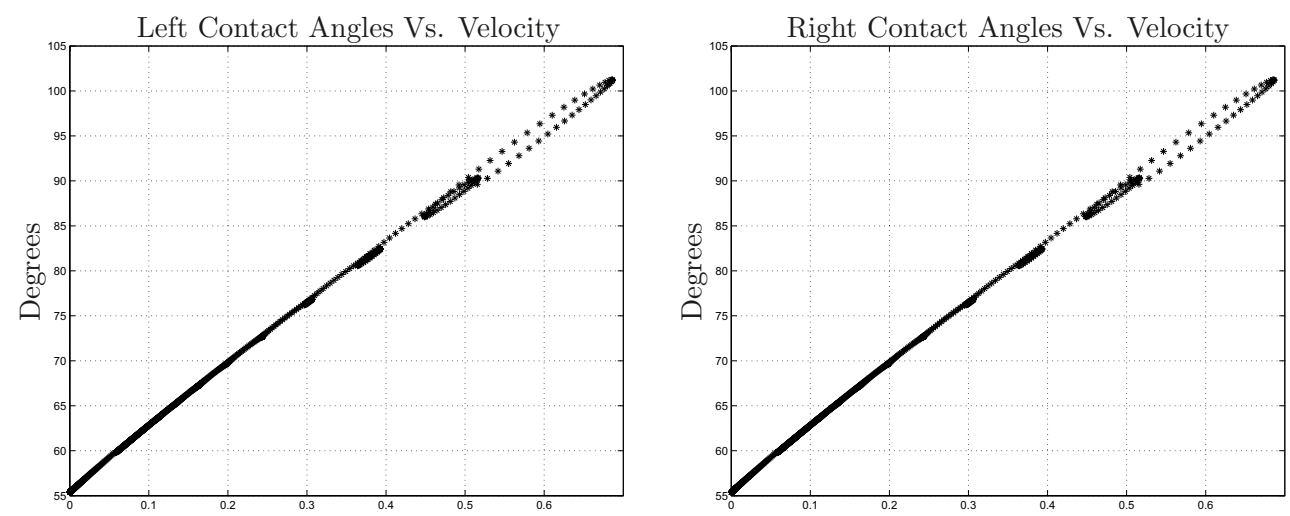

FiguRE 11. Approximately "linear" relationship between contact angle and contact point velocity for simulation in Figure 9. Velocity is in non-dimensional units.

\subsection{Droplet adherence/detachment}

\subsubsection{Wetting regime}

Figure 12 shows a simulation of a droplet pulled upward by gravity (wetting regime). The parameters used are given in Tables 2 and 3, except $\gamma_{\mathrm{s}, \mathrm{g}}$ is changed to a value such that the equilibrium contact angle is $45^{\circ}$, the contact line viscous coefficient is $\beta_{\mathrm{cl}}=1.0 \mathrm{~N} \mathrm{sm}^{-2}$, and $C_{\text {pin }}=0 \mathrm{~N} \mathrm{~m}^{-1}$ (i.e. no pinning). The non-dimensional body force has the form

$$
\mathbf{f}(t)=2.5 \min \left(\frac{t}{0.3}, 1\right) \text { St Ca } \mathbf{e}_{y} .
$$

Figure 13 shows the dynamics of the contact angles $\theta_{\mathrm{cl}}$ and contact points $\mathbf{x}_{\mathrm{cl}}$. Again, the oscillations of the contact angles and contact point velocities are due to the presence of inertia in the fluid. The droplet eventually reaches a stationary configuration with a contact angle of $45^{\circ}$.

\subsubsection{Non-wetting regime}

Figure 14 shows a simulation of a droplet pulled upward by gravity (non-wetting regime). The parameters used are given in Tables 2 and 3 , except $\gamma_{\mathrm{s}, \mathrm{g}}$ is changed to a value such that the equilibrium contact angle 

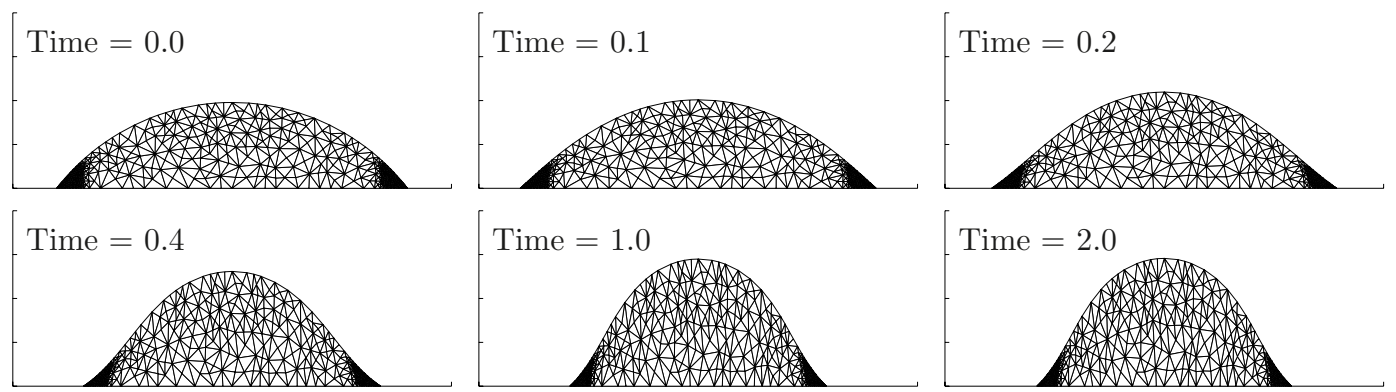

Figure 12. Simulation of a droplet "pulled up" by gravity (wetting regime). The triangulation of $\Omega_{h}$ is shown. Plot window is $[-0.5,1.5] \times[0,0.8]$ in non-dimensional units, and times listed are in non-dimensional units.
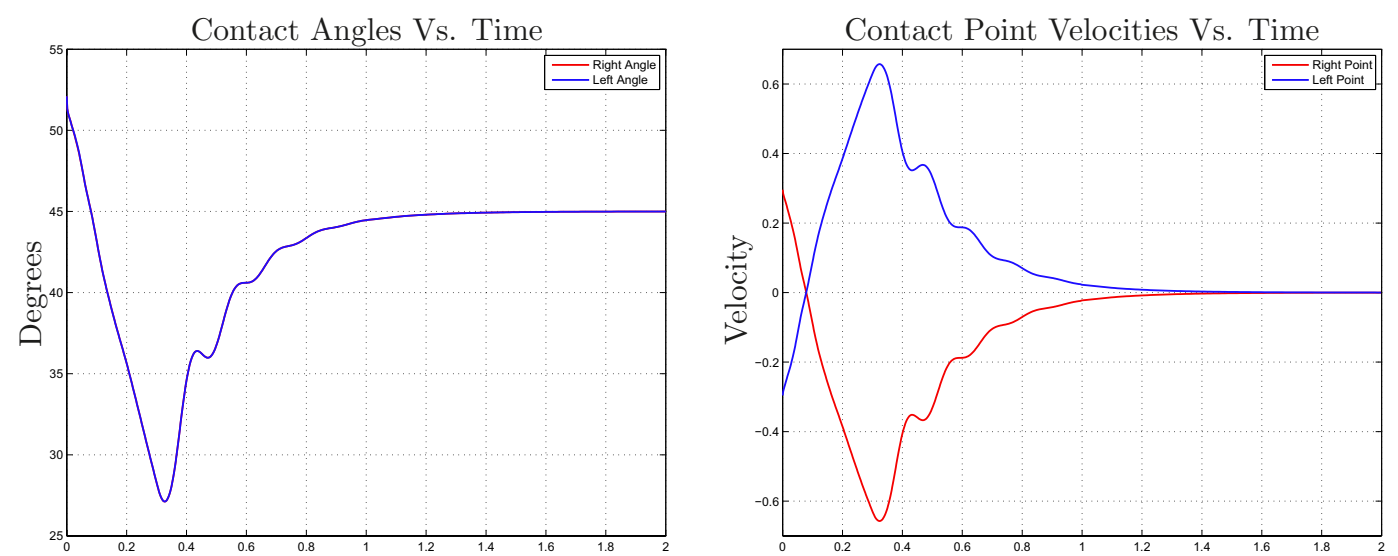

FiguRE 13. Dynamic contact angles and contact points for wetting droplet in Figure 12. The steady-state droplet configuration has a contact angle of $45^{\circ}$. Time and velocity axes are in non-dimensional units.
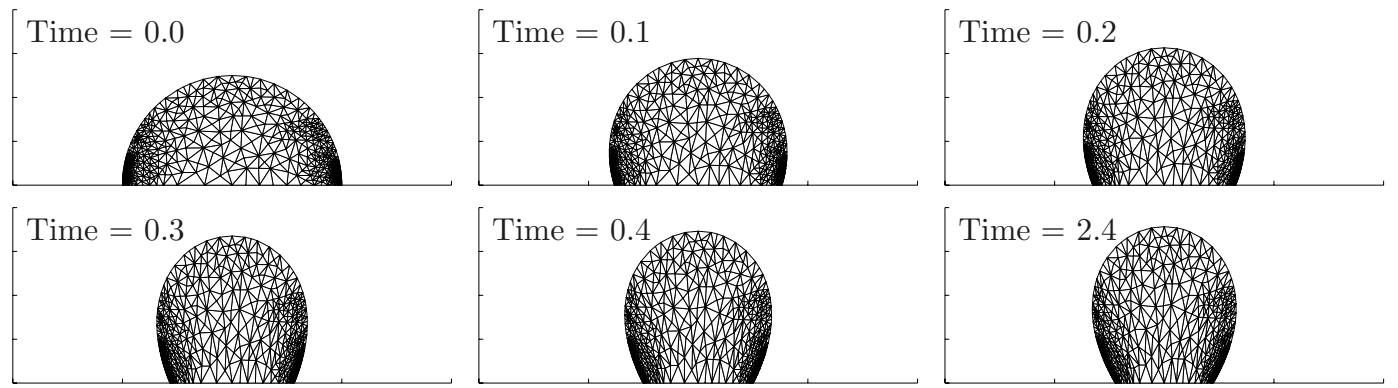

FiguRE 14. Simulation of a droplet "pulled up" by gravity (non-wetting regime). The triangulation of $\Omega_{h}$ is shown. Plot window is $[-0.5,1.5] \times[0,0.8]$ in non-dimensional units, and times listed are in non-dimensional units. 

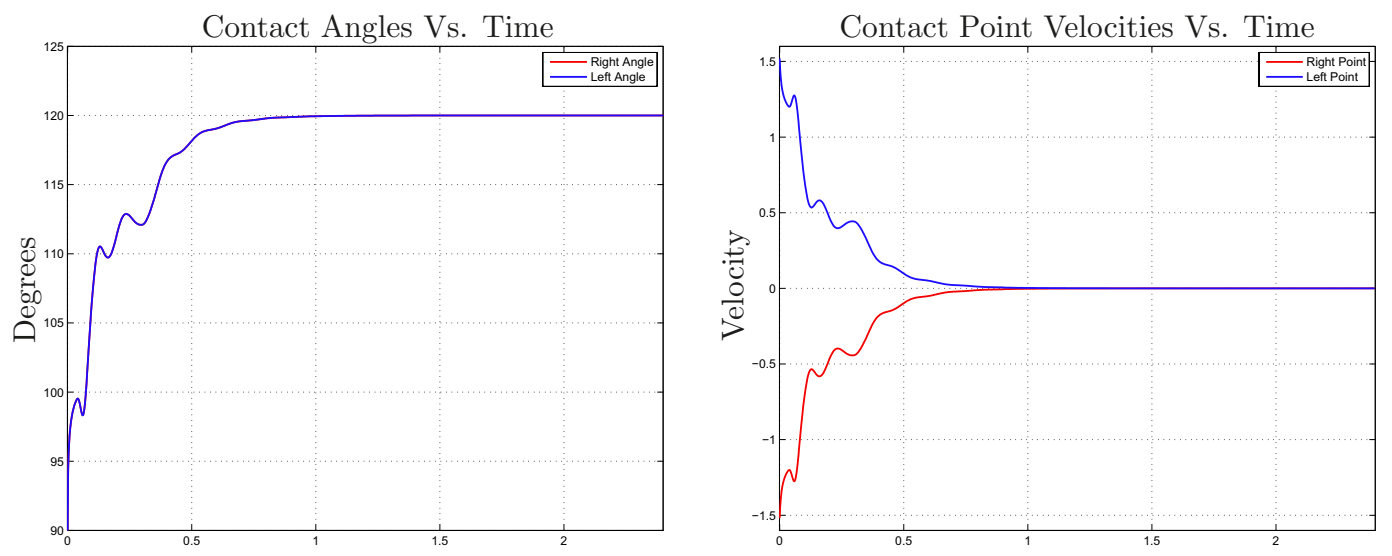

FiguRE 15. Dynamic contact angles and contact points for non-wetting droplet in Figure 14. The steady-state droplet configuration has a contact angle of $120^{\circ}$. Time and velocity axes are in non-dimensional units.

is $120^{\circ}$, the contact line viscous coefficient is $\beta_{\mathrm{cl}}=1.0 \mathrm{~N} \mathrm{sm}^{-2}$, and $C_{\mathrm{pin}}=0 \mathrm{~N} \mathrm{~m}^{-1}$ (i.e. no pinning). The non-dimensional body force has the form

$$
\mathbf{f}(t)=\min \left(\frac{t}{0.3}, 1\right) \operatorname{St~Ca} \mathbf{e}_{y}
$$

Figure 15 shows the dynamics of the contact angles $\theta_{\mathrm{cl}}$ and contact points $\mathbf{x}_{\mathrm{cl}}$. Again, the oscillations of the contact angles and contact point velocities are due to the presence of inertia in the fluid. The droplet eventually reaches a stationary configuration with a contact angle of $120^{\circ}$. Note that increasing the body force would cause the area of the liquid-solid interface to decrease further and eventually lead to the droplet detaching from the solid surface.

\section{Conclusion}

The method presented here offers a well-posed, robust way of modeling flows with moving contact lines. Our methodology provides a framework for including additional physics, such as a soft/elastic substrate, electric fields (i.e. electrowetting $[18,45,70-72]$ ), thermal effects, etc. This simply requires adding terms to the free energy and choosing an appropriate dissipation functional.

The model is easily extended to $3-\mathrm{D}$; in particular, the pinning relation becomes

$$
\lambda=\bar{C}_{\mathrm{pin}} \operatorname{sgn}\left(\left.\dot{\mathbf{X}} \cdot \boldsymbol{\nu}_{\mathrm{cl}}\right|_{\mathbf{x}_{\mathrm{cl}}}\right),
$$

where $\boldsymbol{\nu}_{\mathrm{cl}}$ is the unit normal vector to the 1-D contact line $\partial \Gamma_{\mathrm{g}}$ in the plane of the solid substrate. Most of the analysis does not change much for the 3-D case. In particular, Proposition 3.1 and Lemma 3.2 can be suitably modified. The continuity, coercivity, and inf-sup conditions can also be modified, accounting for the fact that $\mathbf{X}$ is in $H^{1 / 2}\left(\partial \Gamma_{\mathrm{g}}\right)$ which implies that $\lambda$ is in $\left(H^{1 / 2}\left(\partial \Gamma_{\mathrm{g}}\right)\right)^{*}$. Thus, the details of the construction in Lemmas 3.11 and 4.10 change, but the essential idea is the same. But the error analysis is more difficult because the variational inequality is posed in a function space defined on $\partial \Gamma_{\mathrm{g}}$, i.e. we must deal with the approximation of the variational inequality. Moreover, solving the discrete system is harder because $\lambda_{h}$ is high dimensional, so using the projection property (6.3) may not be the most efficient solution method. 
In addition, generating a mesh that conforms to the contact line, and deforming the mesh, introduces some complications. Dealing with topological changes further compounds the problem, but some possible remedies exist; see [49] for a 2-D method. However, there are many industrial applications of wetting with contact lines that do not involve topological changes. Another extension is to consider solid substrates with rigid corners and edges. This will reduce the regularity of the fluid velocity field near the corners of the substrate. Furthermore, the motion of the contact line over a substrate edge will not be trivial. Clearly, this will require adapted meshes near the contact line.

\section{REFERENCES}

[1] R.A. Adams and J.J.F. Fournier, Sobolev Spaces, vol. 140 of Pure Appl. Math. Series, 2nd edn. Elsevier (2003).

[2] V.I. Arnold, Lectures on Partial Differential Equations. Springer (2006).

[3] J.-P. Aubin, Behavior of the error of the approximate solutions of boundary value problems for linear elliptic operators by gelerkin's and finite difference methods. Ann. Scuola Norm. Sup. Pisa 21 (1967) 599-637.

[4] T.A. Baer, R.A. Cairncross, P.R. Schunk, R.R. Rao and P.A. Sackinger, A finite element method for free surface flows of incompressible fluids in three dimensions. Part II. Dynamic wetting lines. Int. J. Numer. Methods Fluids 33 (2000) $405-427$.

[5] E. Bänsch, Finite element discretization of the navier-stokes equations with a free capillary surface. Numer. Math. 88 (2001) 203-235.

[6] E. Bänsch and K. Deckelnick, Optimal error estimates for the stokes and navier-stokes equations with slip-boundary condition. ESAIM: M2AN 33 (1999) 923-938.

[7] E. Bänsch and B. Höhn, Numerical treatment of the navier-stokes equations with slip boundary condition. SIAM J. Sci. Comput. 21 (2000) 2144-2162.

[8] F.B. Belgacem, The Mortar finite element method with Lagrange multipliers. Numer. Math. 84 (1999) $173-197$.

[9] T.D. Blake, The physics of moving wetting lines. J. Colloid Interface Sci. 299 (2006) 1-13.

[10] T.D. Blake and Y.D. Shikhmurzaev, Dynamic wetting by liquids of different viscosity. J. Colloid Interface Sci. 253 (2002) 196-202.

[11] D. Braess, Finite Elements: Theory, Fast Solvers, and Applications in Solid Mechanics, 2nd edn. Cambridge University Press (2001).

[12] S.C. Brenner and L.R. Scott, The Mathematical Theory of Finite Element Methods, 2nd edn. Springer, New York (2002).

[13] F. Brezzi and M. Fortin, Mixed and Hybrid Finite Element Methods. Springer-Verlag, New York (1991).

[14] F. Brezzi, W.W. Hager and P.A. Raviart, Error estimates for the finite element solution of variational inequalities: Part II. Mixed methods. Num. Math. 31 (1978) 1-16.

[15] C.E. Brown, T.D. Jones and E.L. Neustadter, Interfacial flow during immiscible displacement. J. Colloid Interface Sci. 76 (1980) 582-586.

[16] R. Burridge and J.B. Keller, Peeling, slipping and cracking-some one-dimensional free-boundary problems in mechanics. SIAM Review 20 (1978) 31-61.

[17] C.H.A. Cheng, D. Coutand and S. Shkoller, Navier-stokes equations interacting with a nonlinear elastic biofluid shell. SIAM J. Math. Anal. 39 (2007) 742-800.

[18] S.K. Cho, H. Moon and C.-J. Kim, Creating, transporting, cutting, and merging liquid droplets by electrowetting-based actuation for digital microfluidic circuits. J. Microelectromech. Systems 12 (2003) 70-80.

[19] P. Ciarlet, On korns inequality. Chin. Ann. Math. Ser. B 31 (2010) 607-618.

[20] P. Clément, Approximation by finite element functions using local regularization. R.A.I.R.O. Analyse Numérique 9 (1975) 77-84.

[21] P.-P. Cortet, M. Ciccotti and L. Vanel, Imaging the stickslip peeling of an adhesive tape under a constant load. J. Stat. Mech. 2007 (2007) P03005.

[22] J. Cui, X. Chen, F. Wang, X. Gong and Z. Yu, Study of liquid droplets impact on dry inclined surface. Asia-Pacific J. Chem. Eng. 4 (2009) 643-648.

[23] M.C. Delfour and J.-P. Zolésio, Shapes and Geometries: Analysis, Differential Calculus, and Optimization. Vol. 4 of Adv. Des. Control. SIAM (2001).

[24] T. Deng, K. Varanasi, M. Hsu, N. Bhate, C. Keimel, J. Stein and M. Blohm, Non-wetting of impinging droplets on textured surface. Appl. Phys. Lett. 94 (2009) 133109.

[25] S. Dodds, M.S. Carvalho and S. Kumar, The dynamics of three-dimensional liquid bridges with pinned and moving contact lines. J. Fluid Mech. $\mathbf{7 0 7}$ (2012) 521-540.

[26] G. Duvaut and J.L. Lions, Inequalities in Mechanics and Physics. Springer, New York (1976).

[27] C. Eck, M. Fontelos, G. Grün, F. Klingbeil and O. Vantzos, On a phase-field model for electrowetting. Interf. Free Bound. 11 (2009) 259-290.

[28] J. Eggers and R. Evans, Comment on dynamic wetting by liquids of different viscosity, by t.d. blake and y.d. shikhmurzaev. J. Colloid Interf. Sci. 280 (2004) 537-538.

[29] R. Eley and L. Schwartz, Interaction of rheology, geometry, and process in coating flow. J. Coat. Technol. 74 (2002) 43-53. DOI : $10.1007 / \mathrm{BF} 02697974$. 
[30] M.S. Engelman, R.L. Sani and P.M. Gresho, The implementation of normal and/or tangential boundary conditions in finite element codes for incompressible fluid flow. Int. J. Numer. Methods Fluids 2 (1982) 225-238.

[31] L.C. Evans, Partial Differential Equations. American Mathematical Society, Providence, Rhode Island (1998).

[32] R.S. Falk and S.W. Walker, A mixed finite element method for ewod that directly computes the position of the moving interface. SIAM J. Numer. Anal. 51 (2013) 1016-1040.

[33] E. Fermi, Thermodynamics. Dover (1956).

[34] M. Fontelos, G. Grün and S. Jörres, On a phase-field model for electrowetting and other electrokinetic phenomena. SIAM J. Math. Anal. 43 (2011) 527-563.

[35] G.P. Galdi, An introduction to the mathematical theory of the Navier-Stokes equations. I. Linearized steady problems. Vol. 38 of Springer Tracts in Natural Philosophy. Springer-Verlag, New York (1994).

[36] J.-F. Gerbeau and T. Lelièvre, Generalized navier boundary condition and geometric conservation law for surface tension. Comput. Methods Appl. Mech. Eng. 198 (2009) 644-656.

[37] C.M. Groh and M.A. Kelmanson, Multiple-timescale asymptotic analysis of transient coating flows. Phys. Fluids 21 (2009) 091702 .

[38] B. Guo and C. Schwab, Analytic regularity of stokes flow on polygonal domains in countably weighted sobolev spaces. J. Comput. Appl. Math. 190 (2006) 487-519.

[39] K.K. Haller, Y. Ventikos, D. Poulikakos and P. Monkewitz, Computational study of high-speed liquid droplet impact. J. Appl. Phys. 92 (2002) 2821-2828.

[40] J. Haslinger and R.A.E. Mäkinen, Introduction to Shape Optimization: Theory, Approximation, and Computation. Vol. 7 of Adv. Des. Control. SIAM (2003).

[41] C. Huh and L.E. Scriven, Hydrodynamic model of steady movement of a solid/liquid/fluid contact line. J. Colloid Interf. Sci. 35 (1971) 85-101.

[42] Y. Hyon, D.Y. Kwak and C. Liu, Energetic variational approach in complex fluids: Maximum dissipation principle. Discrete Contin. Dyn. Syst. Ser. A 26 (2010) 1291-1304.

[43] M. Lenoir, Optimal isoparametric finite elements and error estimates for domains involving curved boundaries. SIAM J. Numer. Anal. 23 (1986) 562-580.

[44] J.L. Lions and E. Magenes, Non-Homogeneous Boundary Value Problems, Vol. 1. Springer (1972).

[45] F. Mugele and J.-C. Baret, Electrowetting: from basics to applications. J. Phys.: Condensed Matter 17 (2005) R705-R774.

[46] J. Nam and M.S. Carvalho, Mid-gap invasion in two-layer slot coating. J. Fluid Mech. 631 (2009) 397-417.

[47] J. Nitsche, Ein kriterium für die quasi-optimalität des ritzschen verfahrens. Numer. Math. 11 (1968) $346-348$.

[48] R.H. Nochetto, A.J. Salgado and S.W. Walker, A diffuse interface model for electrowettng with moving contact lines. Submitted (2012).

[49] R.H. Nochetto and S.W. Walker, A hybrid variational front tracking-level set mesh generator for problems exhibiting large deformations and topological changes. J. Comput. Phys. 229 (2010) 6243-6269.

[50] L. Onsager, Reciprocal relations in irreversible processes. I. Phys. Rev. 37 (1931) 405-426.

[51] L. Onsager, Reciprocal relations in irreversible processes. II. Phys. Rev. 38 (1931) 2265-2279.

[52] M. Orlt and A.-M. Sändig, Boundary Value Problems And Integral Equations In Nonsmooth Domains, chapter Regularity Of Viscous Navier-Stokes Flows In Nonsmooth Domains. Marcel Dekker, New York (1995) 185-201.

[53] R.F. Probstein, Physicochemical Hydrodynamics: An Introduction, 2nd edn. John Wiley and Sons, Inc., New York (1994).

[54] T. Qian, X.-P. Wang and P. Sheng, Generalized navier boundary condition for the moving contact line. Commun. Math. Sci. 1 (2003) 333-341.

[55] T. Qian, X.-P. Wang and P. Sheng, A variational approach to moving contact line hydrodynamics. J. Fluid Mech. 564 (2006) $333-360$.

[56] W. Ren and W.E., Boundary conditions for the moving contact line problem. Phys. Fluids 19 (2007) 022101.

[57] W. Ren, D. Hu and W.E., Continuum models for the contact line problem. Phys. Fluids 22 (2010) 102103.

[58] R.V. Roy, A.J. Roberts and M.E. Simpson, A lubrication model of coating flows over a curved substrate in space. J. Fluid Mech. 454 (2002) 235-261.

[59] L.R. Scott and S. Zhang, Finite element interpolation of nonsmooth functions satisfying boundary conditions. Math. Comput. 54 (1990) 483-493.

[60] Y.D. Shikhmurzaev, Capillary Flows with Forming Interfaces. Chapman \& Hall/CRC, Boca Raton, FL, 1st edition (2007).

[61] Y.D. Shikhmurzaev and T.D. Blake, Response to the comment on [J. Colloid Interface Sci. 253 (2002) 196] by j. eggers and r. evans. J. Colloid Interf. Sci. 280 (2004) 539-541.

[62] D.N. Sibley, N. Savva and S. Kalliadasis, Slip or not slip? A methodical examination of the interface formation model using two-dimensional droplet spreading on a horizontal planar substrate as a prototype system. Phys. Fluids 24 (2012).

[63] L. Slimane, A. Bendali and P. Laborde, Mixed formulations for a class of variational inequalities. ESAIM: M2AN 38 (2004) $177-201$.

[64] J. Sokolowski and J.-P. Zolésio, Introduction to Shape Optimization. Springer Ser. Comput. Math. Springer-Verlag (1992).

[65] E. Stein, R. de Borst and T.J. Hughes, Encyclopedia of Computational Mechanics. 1 - Fundamentals. Wiley, 1st edition (2004).

[66] R. Temam, Navier-Stokes Equations. Theory and numerical analysis, Reprint of the 1984 edition. AMS Chelsea Publishing, Providence, RI (2001). 
[67] E. Vandre, M.S. Carvalho and S. Kumar, Delaying the onset of dynamic wetting failure through meniscus confinement. J. Fluid Mech. 707 (2012) 496-520.

[68] W. Velte and P. Villaggio, On the detachment of an elastic body bonded to a rigid support. J. Elasticity 27 (1992) $133-142$. DOI : $10.1007 / \mathrm{BF} 00041646$.

[69] R. Verfürth, Finite element approximation of incompressible navier-stokes equations with slip boundary condition. Numer. Math. 50 (1987) 697-721.

[70] S.W. Walker, A. Bonito and R.H. Nochetto, Mixed finite element method for electrowetting on dielectric with contact line pinning. Interf. Free Bound. 12 (2010) 85-119.

[71] S.W. Walker and B. Shapiro, Modeling the fluid dynamics of electrowetting on dielectric (ewod). J. Microelectromech. Systems 15 (2006) 986-1000.

[72] S.W. Walker, B. Shapiro and R.H. Nochetto, Electrowetting with contact line pinning: Computational modeling and comparisons with experiments. Phys. Fluids 21 (2009) 102103.

[73] S.J. Weinstein and K.J. Ruschak, Coating flows. Ann. Rev. Fluid Mech. 36 (2004) 29-53. 